SUPPORTING INFORMATION

\title{
Rhodium-Mediated Dehydrogenative Borylation-Hydroborylation of Bis(alkyl)alkynes: Intermediates and Mechanism
}

Sheila G. Curto, Miguel A. Esteruelas, ${ }^{*}$ Montserrat Oliván and Enrique Oñate

Departamento de Química Inorgánica - Instituto de Síntesis Química y Catálisis Homogénea (ISQCH) Centro de Innovación en Química Avanzada (ORFEO-CINQA), Universidad de Zaragoza - CSIC, 50009 Zaragoza, Spain

* e-mail: maester@unizar.es

\section{Contents:}

- General Information.

- Structural Analysis of Complexes 9-11 and 14.

- NMR spectra 
General Information. All reactions were carried out with exclusion of air using Schlenk-tube techniques or in a drybox. Pentane and toluene were obtained oxygen- and water-free from an MBraun solvent purification apparatus. $n$-Octane was dried over sodium and distilled prior to use. ${ }^{1} \mathrm{H},{ }^{13} \mathrm{C}\left\{{ }^{1} \mathrm{H}\right\},{ }^{31} \mathrm{P}\left\{{ }^{1} \mathrm{H}\right\}$, and ${ }^{11} \mathrm{~B}\left\{{ }^{1} \mathrm{H}\right\}$ NMR spectra were recorded on Bruker 300 ARX, Bruker Avance $300 \mathrm{MHz}$, Bruker Avance $400 \mathrm{MHz}$ or Bruker Avance $500 \mathrm{MHz}$ instruments. Chemical shifts (expressed in ppm) are referenced to residual solvent peaks $\left({ }^{1} \mathrm{H},{ }^{13} \mathrm{C}\left\{{ }^{1} \mathrm{H}\right\}\right)$, external $85 \% \mathrm{H}_{3} \mathrm{PO}_{4}\left({ }^{31} \mathrm{P}\left\{{ }^{1} \mathrm{H}\right\}\right)$, or $\mathrm{BF}_{3} \cdot \mathrm{OEt}_{2}\left({ }^{11} \mathrm{~B}\left\{{ }^{1} \mathrm{H}\right\}\right)$. Coupling constants $J$ and $N$ are given in hertz. Attenuated total reflection infrared spectra (ATR-IR) of solid samples were run on a PerkinElmer Spectrum 100 FT-IR spectrometer. C, H, and N analyses were carried out in a PerkinElmer $2400 \mathrm{CHNS} / \mathrm{O}$ analyzer. High-resolution electrospray mass spectra were acquired using a MicroTOF-Q hybrid quadrupole time-of-flight spectrometer (Bruker Daltonics, Bremen, Germany).

Structural Analysis of Complexes 9-11 and 14. X-ray data were collected for the complexes on a Bruker Smart APEX diffractometer equipped with a normal focus, and $2.4 \mathrm{~kW}$ sealed tube source (Mo radiation, $\lambda=0.71073 \AA$ ). Data were collected over the complete sphere covering $0.3^{\circ}$ in $\omega$. Data were corrected for absorption by using a multiscan method applied with the SADABS program. ${ }^{1}$ The structures were solved by Patterson or direct methods and refined by full-matrix least squares on $\mathrm{F}^{2}$ with SHELXL2016, ${ }^{2}$ including isotropic and subsequently anisotropic displacement parameters. The hydrogen atoms were observed in the least Fourier Maps or calculated, and refined freely or using a restricted riding model.

The crystal of 9-11 results of a co-crystallization of the E/Z alkenyl ligands in 0.7/0.3 ratio. These ligands were refined with restrained geometry, complementary occupancy factors and isotropic displacement parameters. The pentyl substituent of the allyl ligand in 14 was observed disordered and refined with two moieties, restrained geometry, complementary occupancy factors and isotropic displacement parameters.

Crystal data for 9-11 (CCDC 1886050): $\mathrm{C}_{33} \mathrm{H}_{51} \mathrm{OP}_{2} \mathrm{Rh}, \mathrm{M}_{\mathrm{W}}$ 628.58, red, irregular block $\left(0.214 \times 0.174 \times 0.161 \mathrm{~mm}^{3}\right)$, triclinic, space group P-1, $a$ : 11.7443(6) $\AA, b$ : 12.1790(6) $\AA, c: 13.1365(7) \AA, a: 89.0940(10)^{\circ}, \beta: 73.2720(10)^{\circ}, \gamma: 61.8600(10)^{\circ}, V=$ 1570.67(14) $\AA^{3}, Z=2, Z^{\prime}=1, \mathrm{D}_{\text {calc }}: 1.329 \mathrm{~g} \mathrm{~cm}^{-3}, \mathrm{~F}(000): 664, \mathrm{~T}=100(2) \mathrm{K}, \mu 0.669$ $\mathrm{mm}^{-1} .28195$ measured reflections $\left(2 \theta: 3-57^{\circ}\right.$, $\omega$ scans $\left.0.3^{\circ}\right), 7578$ unique $\left(\mathrm{R}_{\text {int }}=\right.$ 0.0312 ); min./max. transm. factors $0.754 / 0.862$. Final agreement factors were $\mathrm{R}^{1}=$ 
$0.0385(6593$ observed reflections, $\mathrm{I}>2 \sigma \quad(\mathrm{I}))$ and $\mathrm{wR}^{2}=0.0959$; data/restraints/parameters 7578/168/407; $\mathrm{GoF}=1.111$. Largest peak and hole 1.109 (close to rhodium atoms) and $-1.178 \mathrm{e} / \AA^{3}$.

Crystal data for 14 (CCDC 1886051): $\mathrm{C}_{35} \mathrm{H}_{55} \mathrm{OP}_{2} \mathrm{Rh}, 0.5\left(\mathrm{C}_{5} \mathrm{H}_{12}\right), \mathrm{M}_{\mathrm{W}}$ 692.71, orange, irregular block $\left(0.347 \times 0.180 \times 0.108 \mathrm{~mm}^{3}\right)$, triclinic, space group P-1, $a$ : 11.9331(7) $\AA, \quad b: 12.2612(7) \AA, c: 13.8481(8) \AA, a: 116.0490(10)^{\circ}, \beta: 95.4280(10)^{\circ}, \gamma:$ 90.1540(10) ${ }^{\circ}, V=1809.97(18) \AA^{3}, Z=2, Z^{\prime}=1, \mathrm{D}_{\text {calc }}: 1.271 \mathrm{~g} \mathrm{~cm}^{-3}, \mathrm{~F}(000): 738, \mathrm{~T}=$

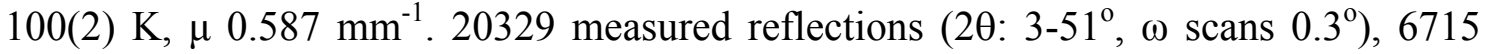
unique $\left(\mathrm{R}_{\text {int }}=0.0273\right)$; min./max. transm. factors $0.757 / 0.862$. Final agreement factors were $\mathrm{R}^{1}=0.0388$ (5971 observed reflections, $\mathrm{I}>2 \sigma(\mathrm{I})$ ) and $\mathrm{wR}^{2}=0.1041$; data/restraints/parameters 6715/21/388; GoF $=1.035$. Largest peak and hole 2.379 (probably due to a residual, $\approx 25 \%$, water solvent molecule) and $-0.702 \mathrm{e} / \AA^{3}$.

(1) Blessing, R. H. Acta Crystallogr. 1995, A51, 33. SADABS: Area-detector absorption correction; Bruker- AXS, Madison, WI, 1996.

(2) SHELXL-2016/6. Sheldrick, G. M. Acta Cryst. 2008, A64, 112-122. 


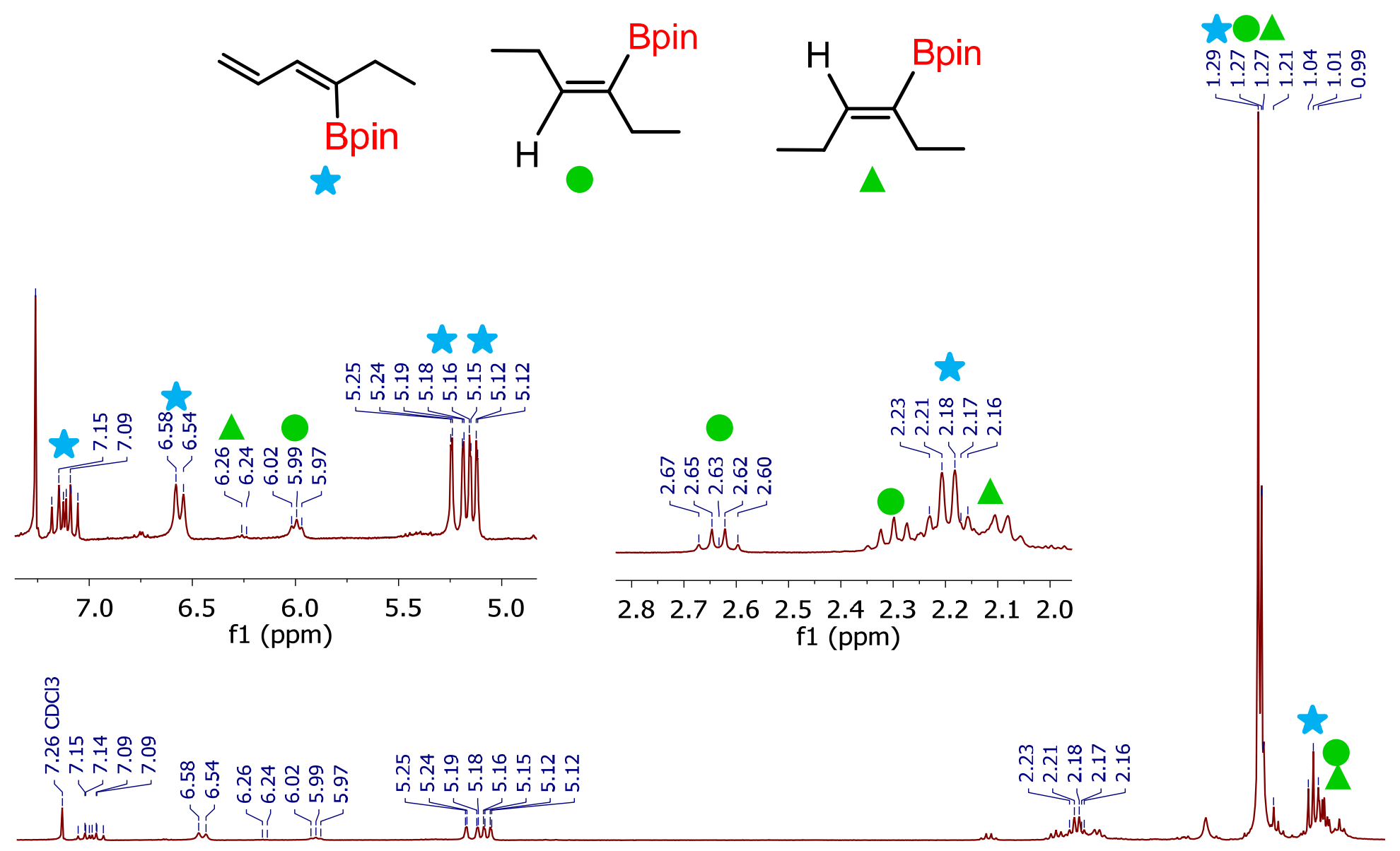

$\begin{array}{lllllllllllllllllll}7.2 & 6.8 & 6.4 & 6.0 & 5.6 & 5.2 & 4.8 & 4.4 & \begin{array}{c}4.0 \\ \mathrm{f} 1(\mathrm{ppm})\end{array} & 3.6 & 3.2 & 2.8 & 2.4 & 2.0 & 1.6 & 1.2 & 0.8\end{array}$

Figure S1. ${ }^{1} \mathrm{H}$ NMR spectrum $\left(300.13 \mathrm{MHz}, \mathrm{CDCl}_{3}, 298 \mathrm{~K}\right)$ of the products (after purification by passing the crude reaction mixture through a short pad of silica gel) of the catalytic borylation of 3-hexyne with $\mathrm{B}_{2} \mathrm{pin}_{2}$ catalyzed by complex 1 . 


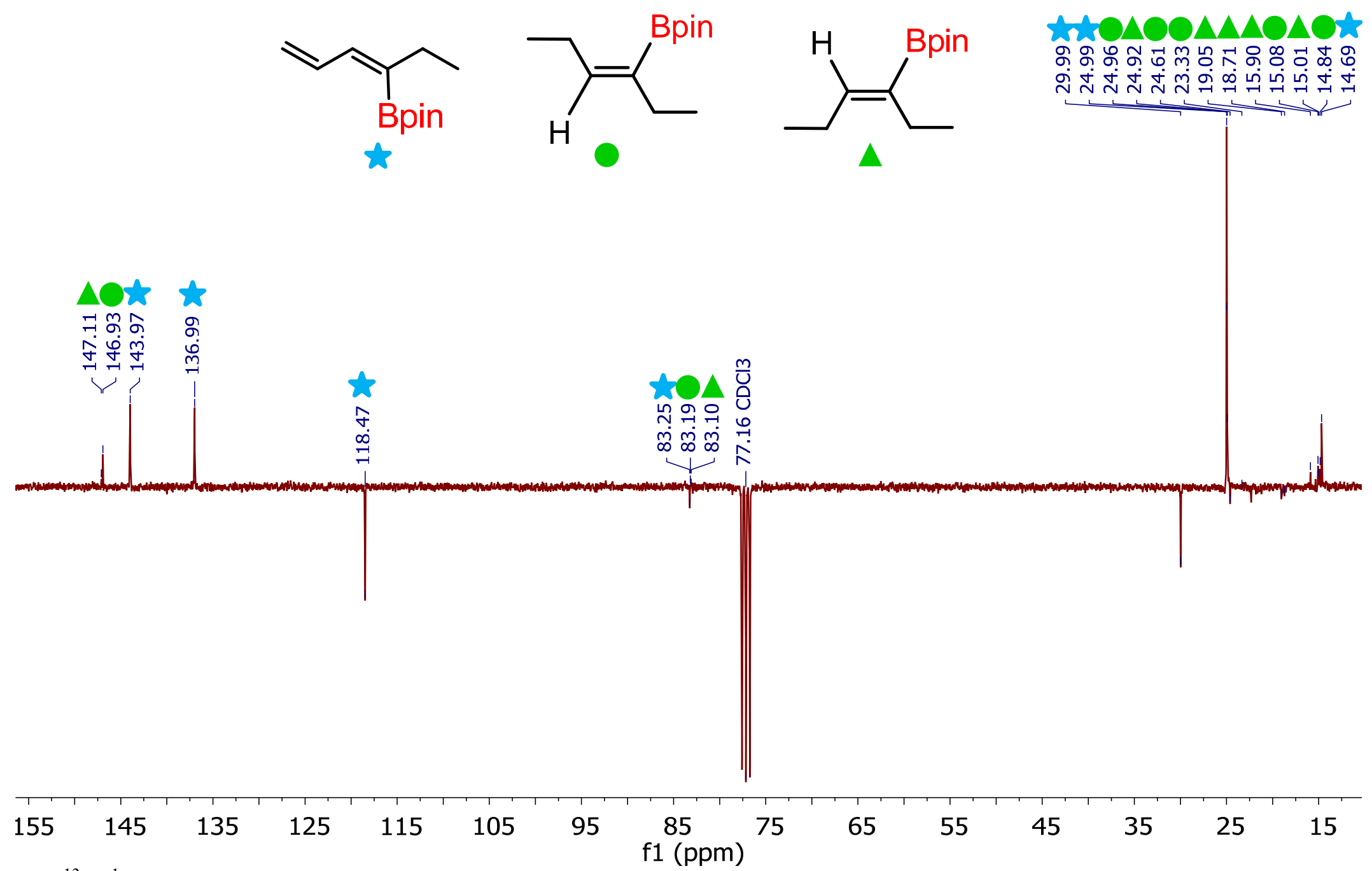

Figure S2. ${ }^{13} \mathrm{C}\left\{{ }^{1} \mathrm{H}\right\}$-apt NMR spectrum $\left(75.48 \mathrm{MHz}, \mathrm{CDCl}_{3}, 298 \mathrm{~K}\right)$ of the products (after purification by passing the crude reaction mixture through a short pad of silica gel) of the catalytic borylation of 3-hexyne with $\mathrm{B}_{2} \mathrm{pin}_{2}$ catalyzed by complex 1. 

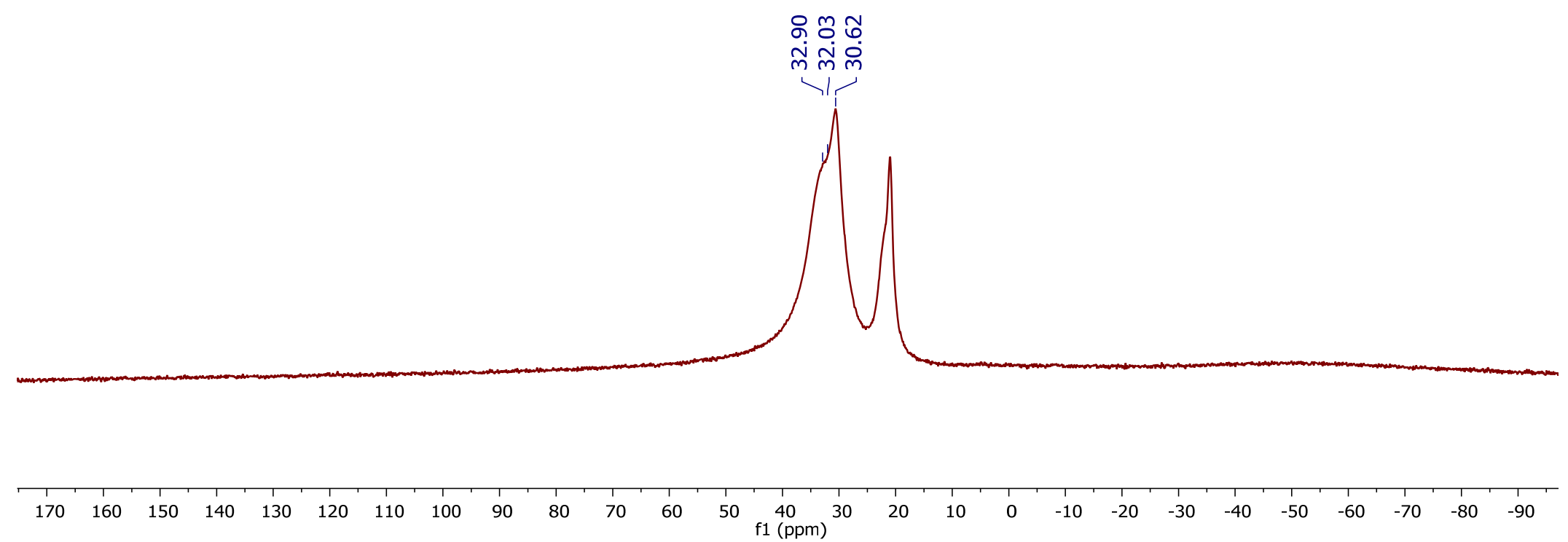

Figure S3. ${ }^{11} \mathrm{~B}$ NMR spectrum $\left(96.29 \mathrm{MHz}, \mathrm{CDCl}_{3}, 298 \mathrm{~K}\right.$ ) of the products (after purification by passing the crude reaction mixture through a short pad of silica gel) of the catalytic borylation of 3-hexyne with $\mathrm{B}_{2} \mathrm{pin}_{2}$ catalyzed by complex 1 . 

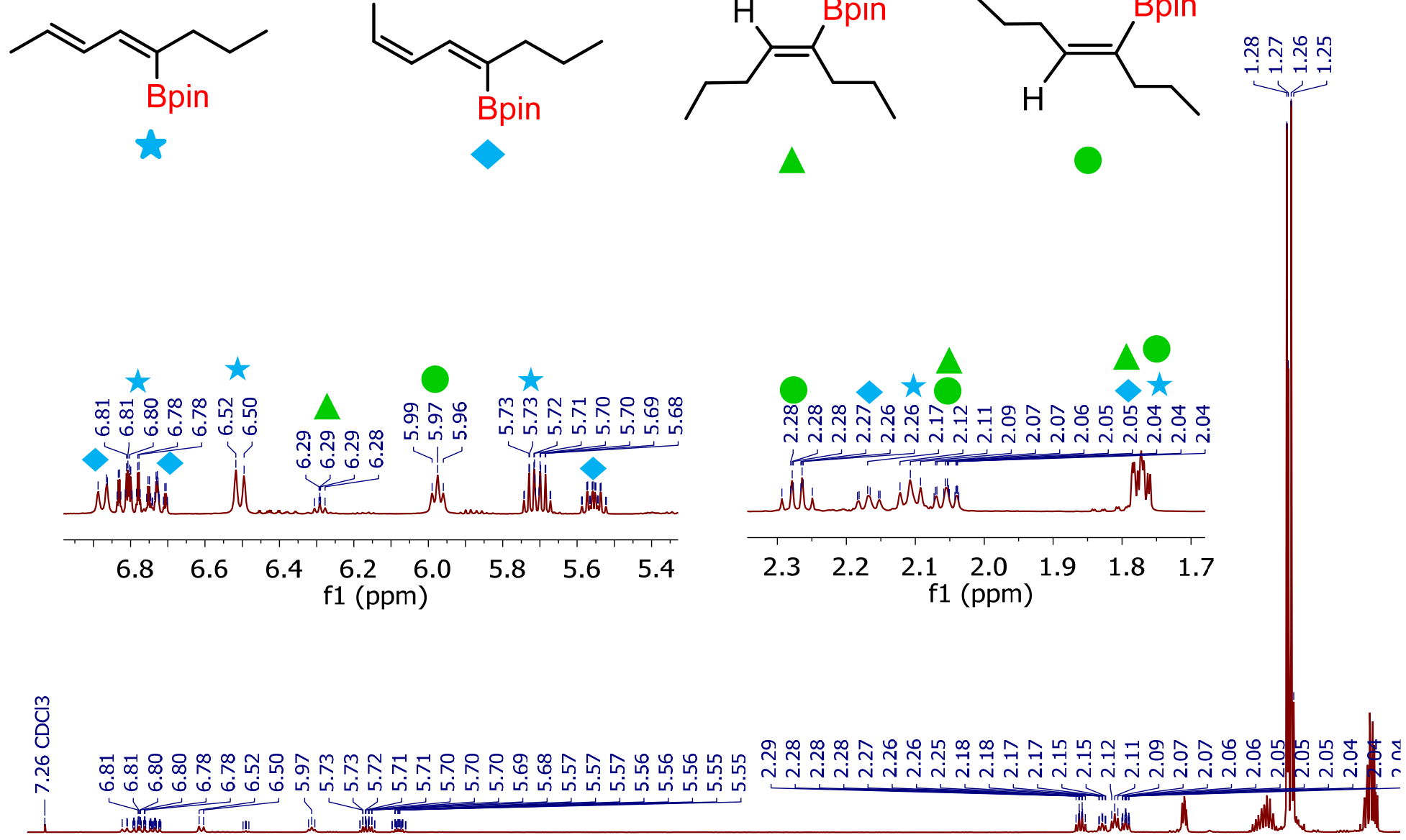

7.2

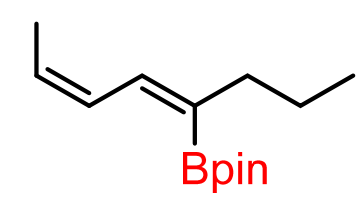

$>$<smiles>CCC/C=C\CCC</smiles>

$\Lambda$

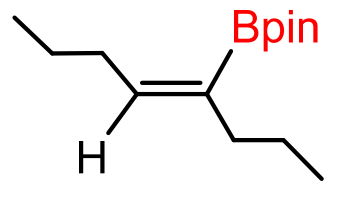

O
ஸึ

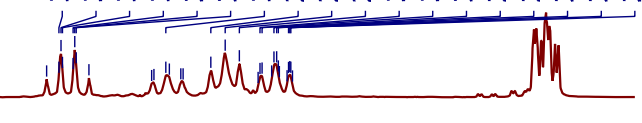

$\begin{array}{lllllll}2.3 & 2.2 & 2.1 & 2.0 & 1.9 & 1.8 & 1.7\end{array}$

f1 (ppm)

$\begin{array}{lllllll}6.8 & 6.4 & 6.0 & 5.6 & 5.2 & 4.8 & 4\end{array}$

$$
\text { f1 (ppm) }
$$

Figure S4. ${ }^{1} \mathrm{H}$ NMR spectrum $\left(500.13 \mathrm{MHz}, \mathrm{CDCl}_{3}, 298 \mathrm{~K}\right.$ ) of the products (after purification by passing the crude reaction mixture through a short pad of silica gel) of the catalytic borylation of 4-octyne with $\mathrm{B}_{2} \mathrm{pin}_{2}$ catalyzed by complex $\mathbf{1}$. 


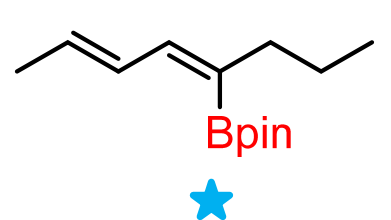

t)

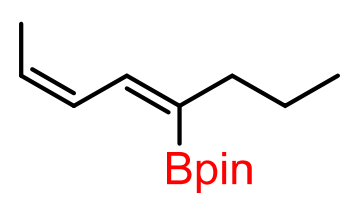

$>$

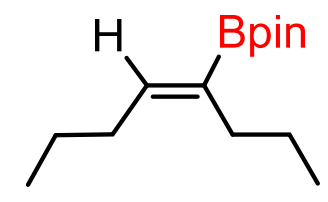

$\triangle$ tod oth ats thoo

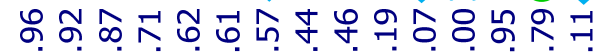
ป்

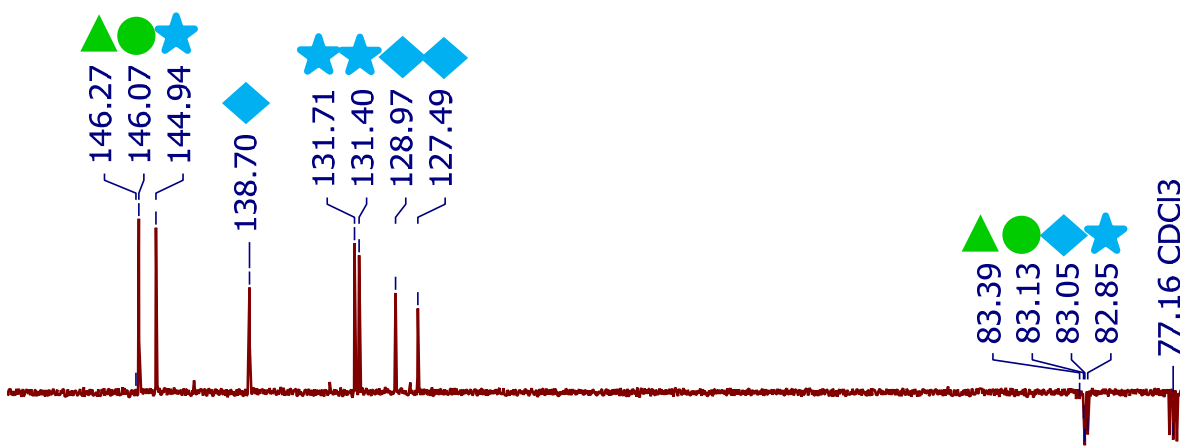<smiles>CCCC=C(Br)Br</smiles>
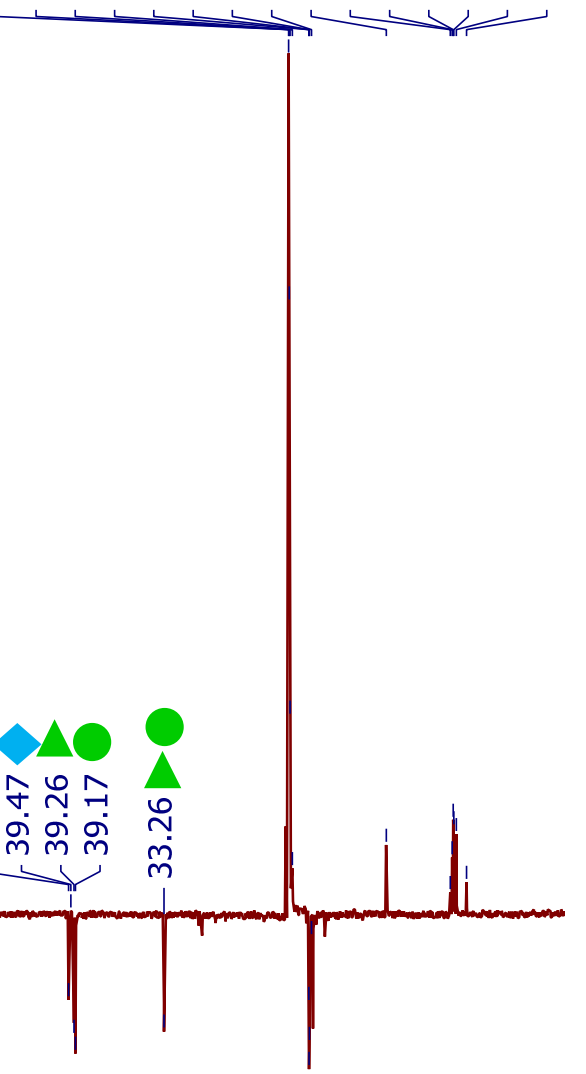

$\begin{array}{llllllllllllllll}150 & 140 & 130 & 120 & 110 & 100 & 90 & \begin{array}{c}80 \\ \mathrm{f} 1(\mathrm{ppm})\end{array} & 70 & 60 & 50 & 40 & 30 & 20 & 10\end{array}$

Figure S5. ${ }^{13} \mathrm{C}\left\{{ }^{1} \mathrm{H}\right\}$-apt NMR spectrum $\left(125.77 \mathrm{MHz}, \mathrm{CDCl}_{3}, 298 \mathrm{~K}\right.$ ) of the products (after purification by passing the crude reaction mixture through a short pad of silica gel) of the catalytic borylation of 4-octyne with $\mathrm{B}_{2} \mathrm{pin}_{2}$ catalyzed by complex 1 . 


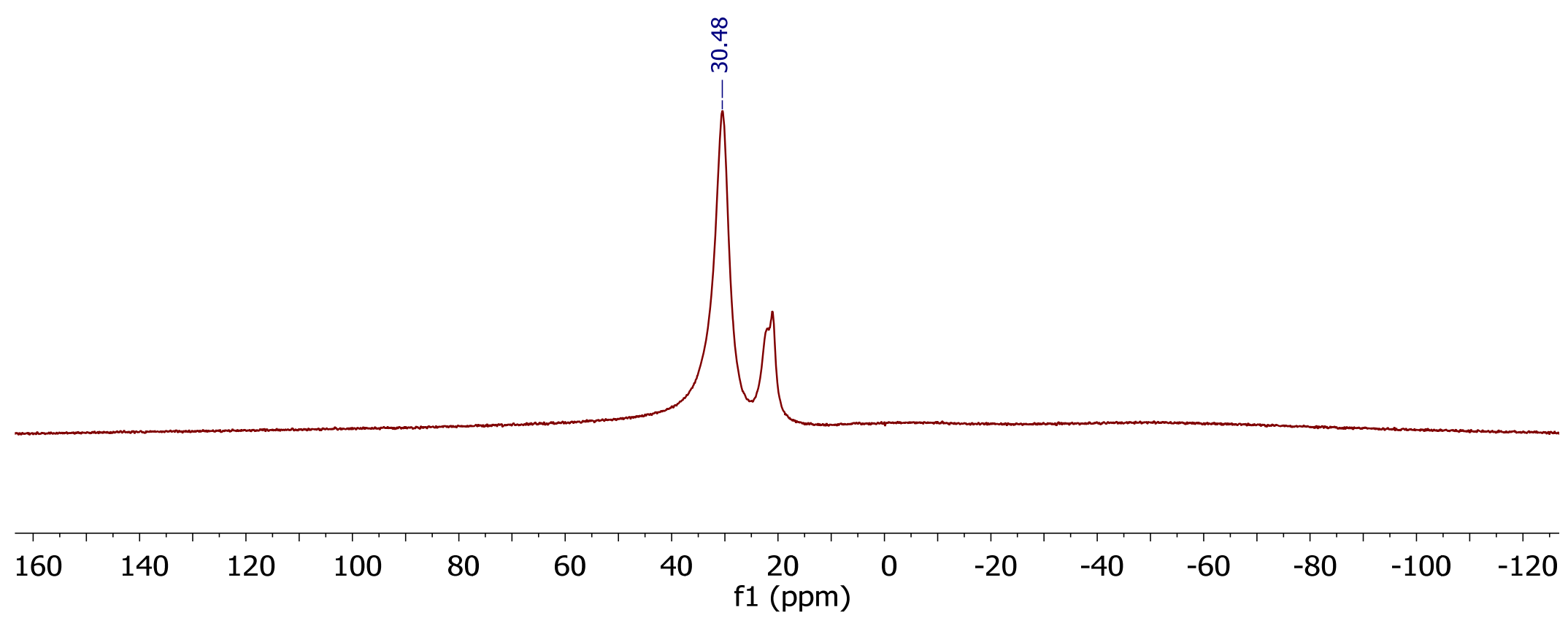

Figure S6. ${ }^{11} \mathrm{~B}$ NMR spectrum $\left(96.29 \mathrm{MHz}, \mathrm{CDCl}_{3}, 298 \mathrm{~K}\right.$ ) of the products (after purification by passing the crude reaction mixture through a short pad of silica gel) of the catalytic borylation of 4-octyne with $\mathrm{B}_{2} \operatorname{pin}_{2}$ catalyzed by complex 1 . 


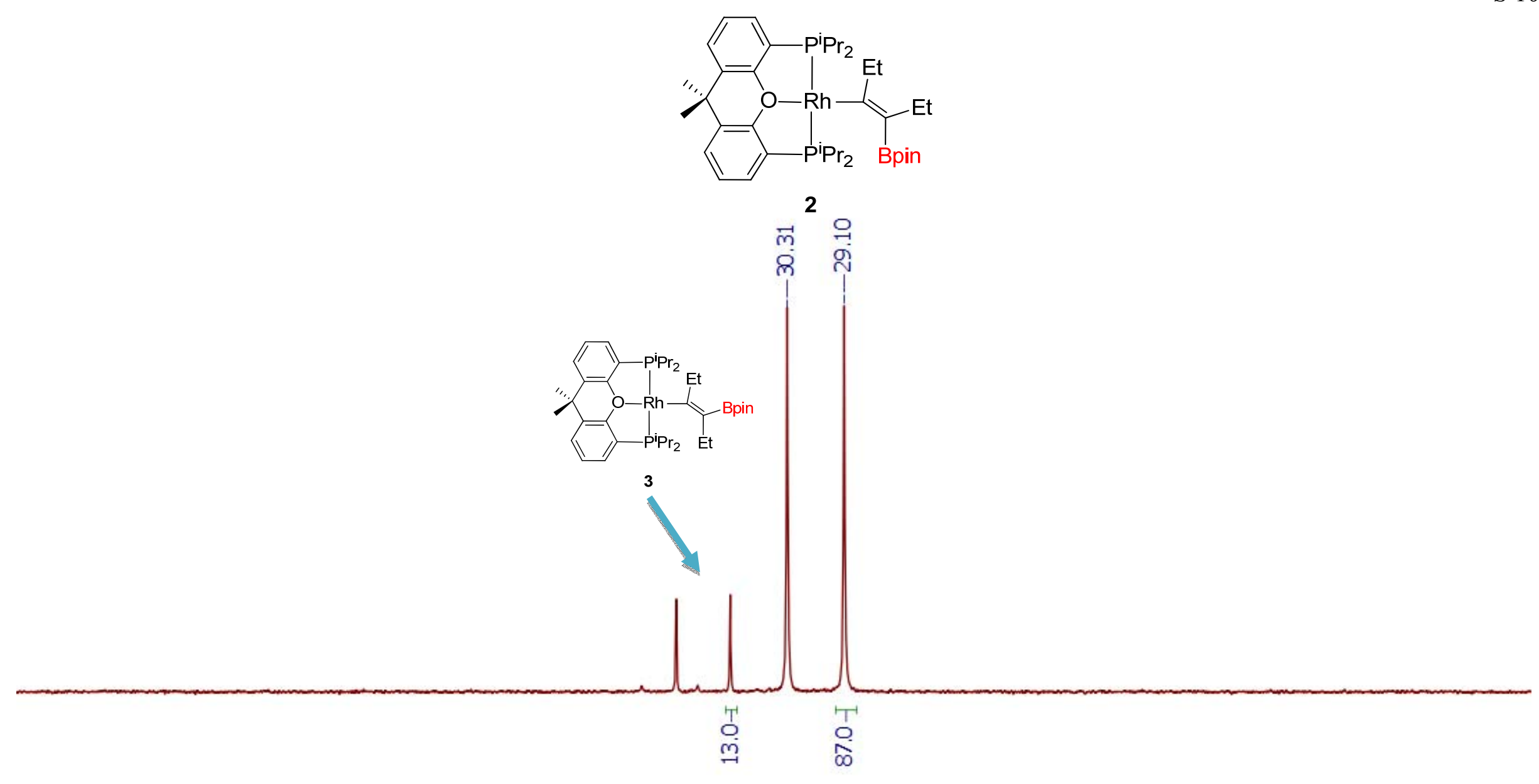

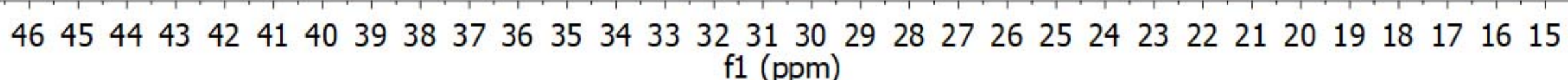

Figure S7. ${ }^{31} \mathrm{P}\left\{{ }^{1} \mathrm{H}\right\}$ NMR spectrum $\left(161.98 \mathrm{MHz}, \mathrm{C}_{7} \mathrm{D}_{8}, 253 \mathrm{~K}\right)$ of the reaction of $\mathrm{Rh}(\mathrm{Bpin})\left\{\kappa^{3}-\mathrm{P}, \mathrm{O}, \mathrm{P}-\left[\mathrm{xant}\left(\mathrm{P}^{\mathrm{i}} \mathrm{Pr}_{2}\right)_{2}\right]\right\}(\mathbf{1})$ with 3-hexyne at low temperature: Spectroscopic detection of $\operatorname{Rh}\{(Z)-\mathrm{C}(\mathrm{Et})=\mathrm{C}(\mathrm{Et}) \mathrm{Bpin})\left\{\kappa^{3}-\mathrm{P}, \mathrm{O}, \mathrm{P}-\left[\operatorname{xant}\left(\mathrm{P}^{\mathrm{i}} \mathrm{Pr}_{2}\right)_{2}\right]\right\}(\mathbf{2})$. 


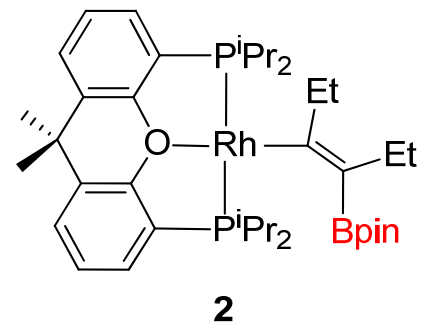

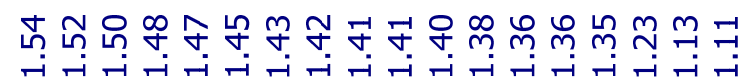

2
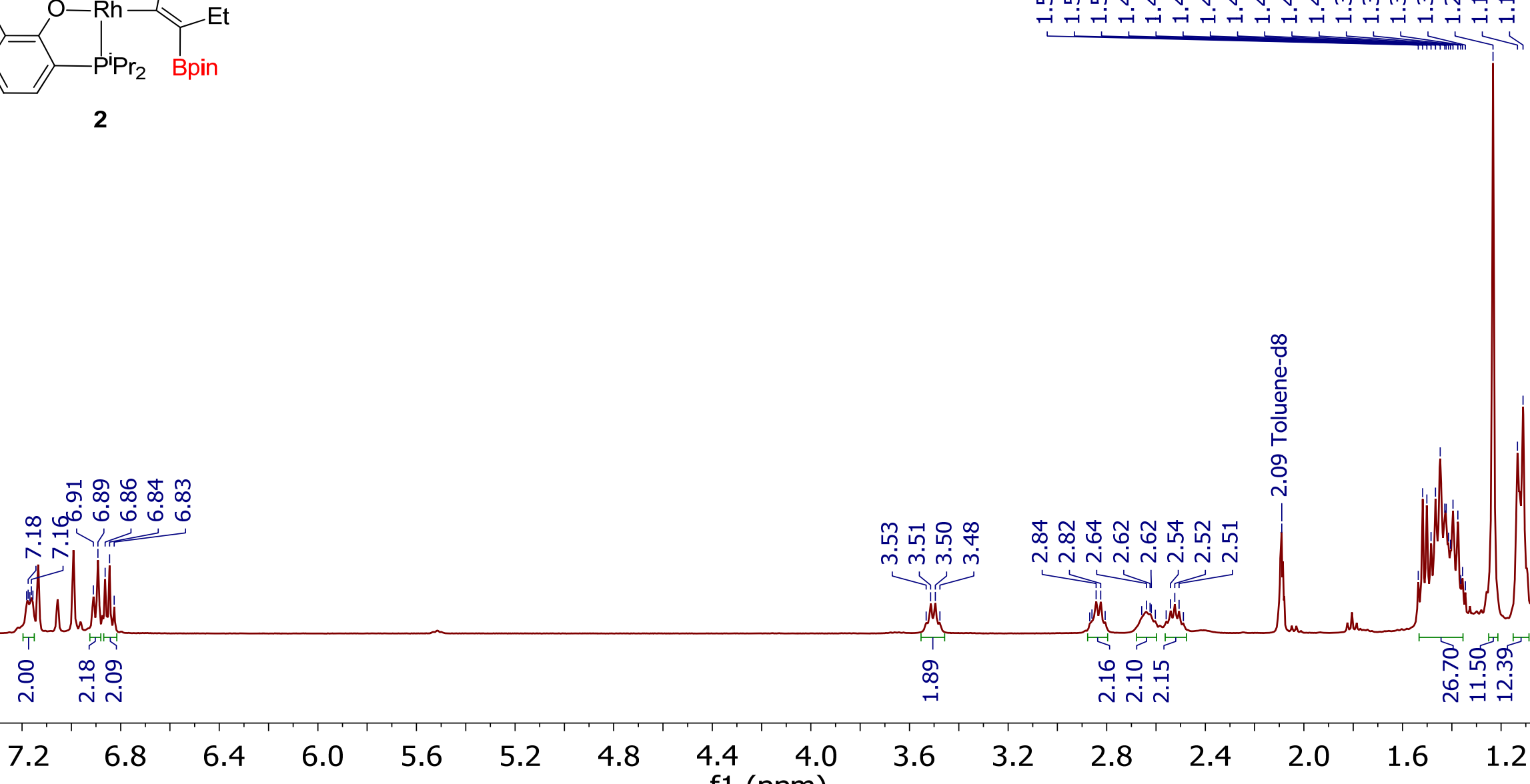

$\begin{array}{llllllll}7.2 & 6.8 & 6.4 & 6.0 & 5.6 & 5.2 & 4.8 & \begin{array}{l}4.4 \\ \mathrm{f} 1(\mathrm{ppm})\end{array}\end{array}$

Figure S8. ${ }^{1} \mathrm{H}$ NMR spectrum $\left(400.13 \mathrm{MHz}, \mathrm{C}_{7} \mathrm{D}_{8}, 253 \mathrm{~K}\right)$ of the reaction of $\mathrm{Rh}(\mathrm{Bpin})\left\{\kappa^{3}-\mathrm{P}, \mathrm{O}, \mathrm{P}-\left[\operatorname{xant}\left(\mathrm{P}^{\mathrm{i}} \mathrm{Pr}_{2}\right)_{2}\right]\right\}(\mathbf{1})$ with 3-hexyne at low temperature: Spectroscopic detection of $\operatorname{Rh}\{(Z)-C(E t)=C(E t) B p i n)\left\{\kappa^{3}-\mathrm{P}, \mathrm{O}, \mathrm{P}-\left[\operatorname{xant}\left(\mathrm{P}^{\mathrm{i}} \mathrm{Pr}_{2}\right)_{2}\right]\right\}$ (2). 


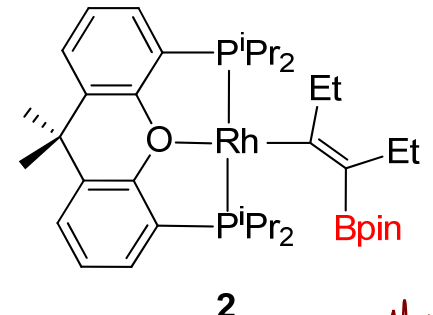

2

\section{๘}

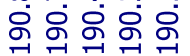

귁ㄱㄱ

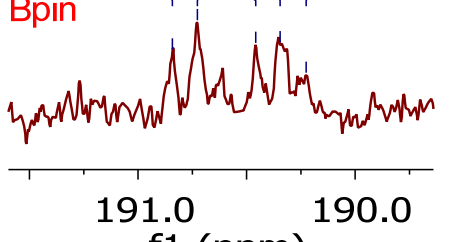

f1 (ppm)
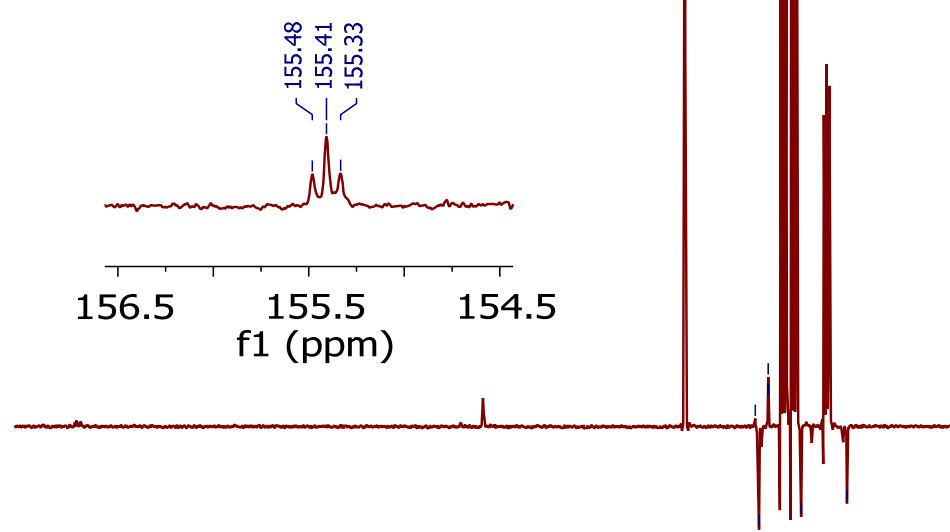

은 m.

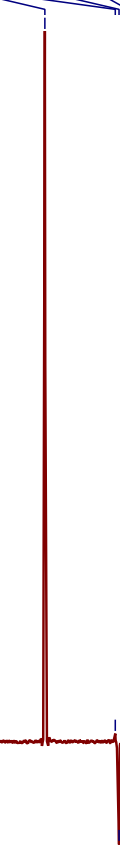

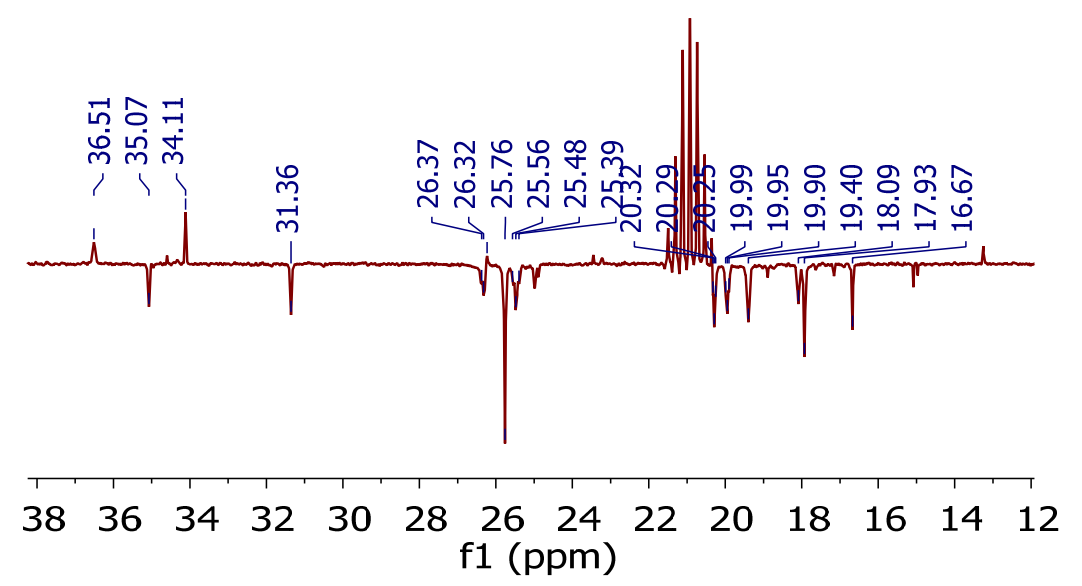

ติ

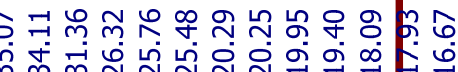

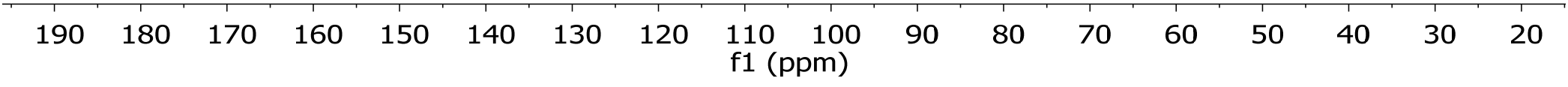

Figure S9. ${ }^{13} \mathrm{C}\left\{{ }^{1} \mathrm{H}\right\}$-apt NMR spectrum $\left(100.62 \mathrm{MHz}, \mathrm{C}_{7} \mathrm{D}_{8}, 253 \mathrm{~K}\right)$ of the reaction of $\mathrm{Rh}(\mathrm{Bpin})\left\{\kappa^{3}-\mathrm{P}, \mathrm{O}, \mathrm{P}-\left[\mathrm{xant}\left(\mathrm{P}^{\mathrm{i}} \mathrm{Pr}_{2}\right)_{2}\right]\right\}(\mathbf{1})$ with 3-hexyne at low temperature: Spectroscopic detection of $\mathrm{Rh}\{(Z)-\mathrm{C}(\mathrm{Et})=\mathrm{C}(\mathrm{Et}) \mathrm{Bpin})\left\{\kappa^{3}-\mathrm{P}, \mathrm{O}, \mathrm{P}-\left[\operatorname{xant}\left(\mathrm{P}^{\mathrm{i}} \mathrm{Pr}_{2}\right)_{2}\right]\right\}$ (2). 


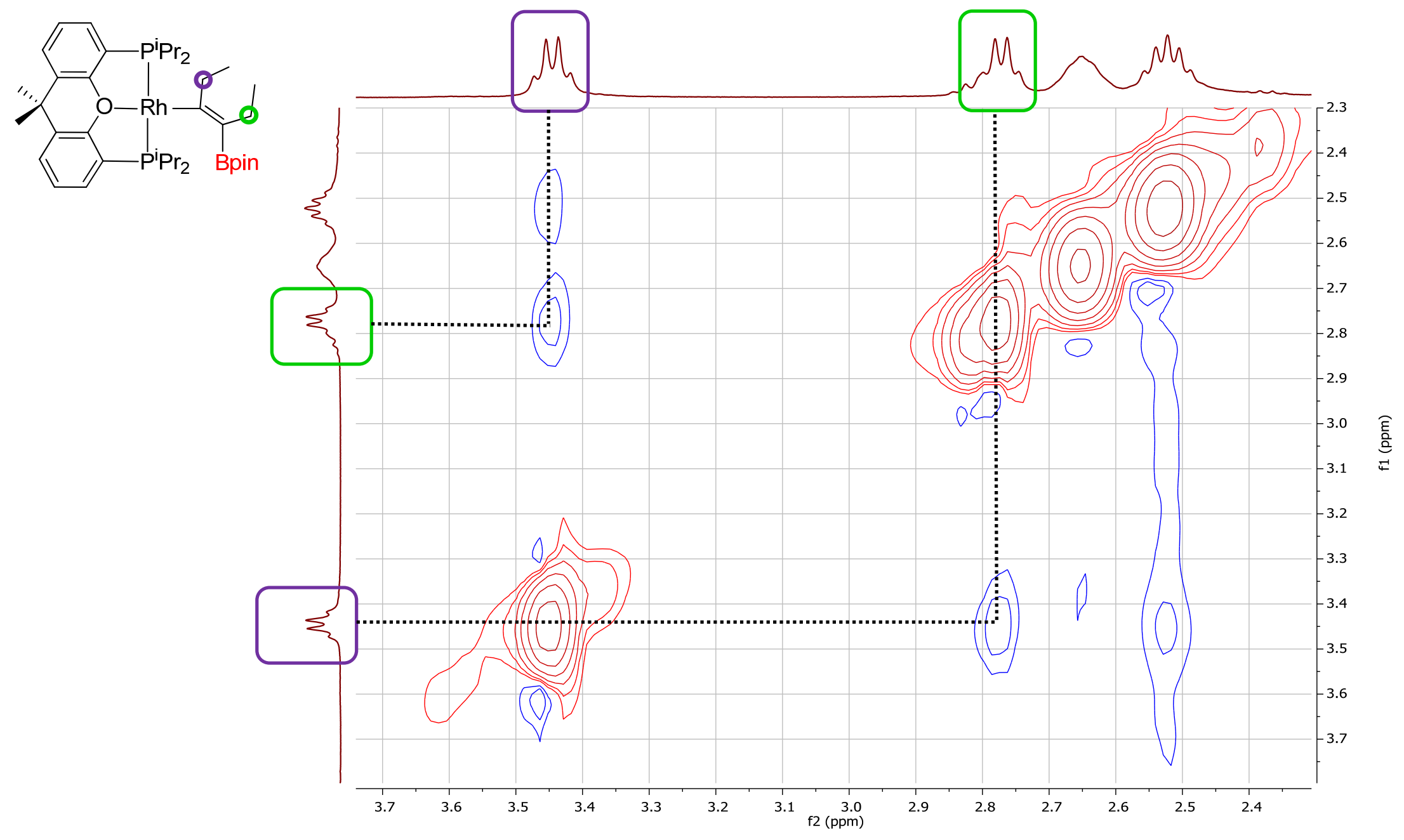

Figure S10. Partial view of the ${ }^{1} \mathrm{H},{ }_{1}^{1} \mathrm{H}$ NOESY NMR spectrum $\left(400.13 \mathrm{MHz}, \mathrm{C}_{7} \mathrm{D}_{8}, 253 \mathrm{~K}\right)$ of $\mathrm{Rh}\{(Z)-\mathrm{C}(\mathrm{Et})=\mathrm{C}(\mathrm{Et}) \mathrm{Bpin}\}\left\{\kappa^{3}-\mathrm{P}, \mathrm{O}, \mathrm{P}-\right.$ $\left.\left[\operatorname{xant}\left(\mathrm{P}^{\mathrm{i}} \mathrm{Pr}_{2}\right)_{2}\right]\right\}(2)$. 


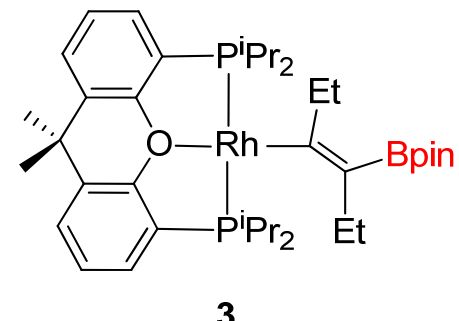

3

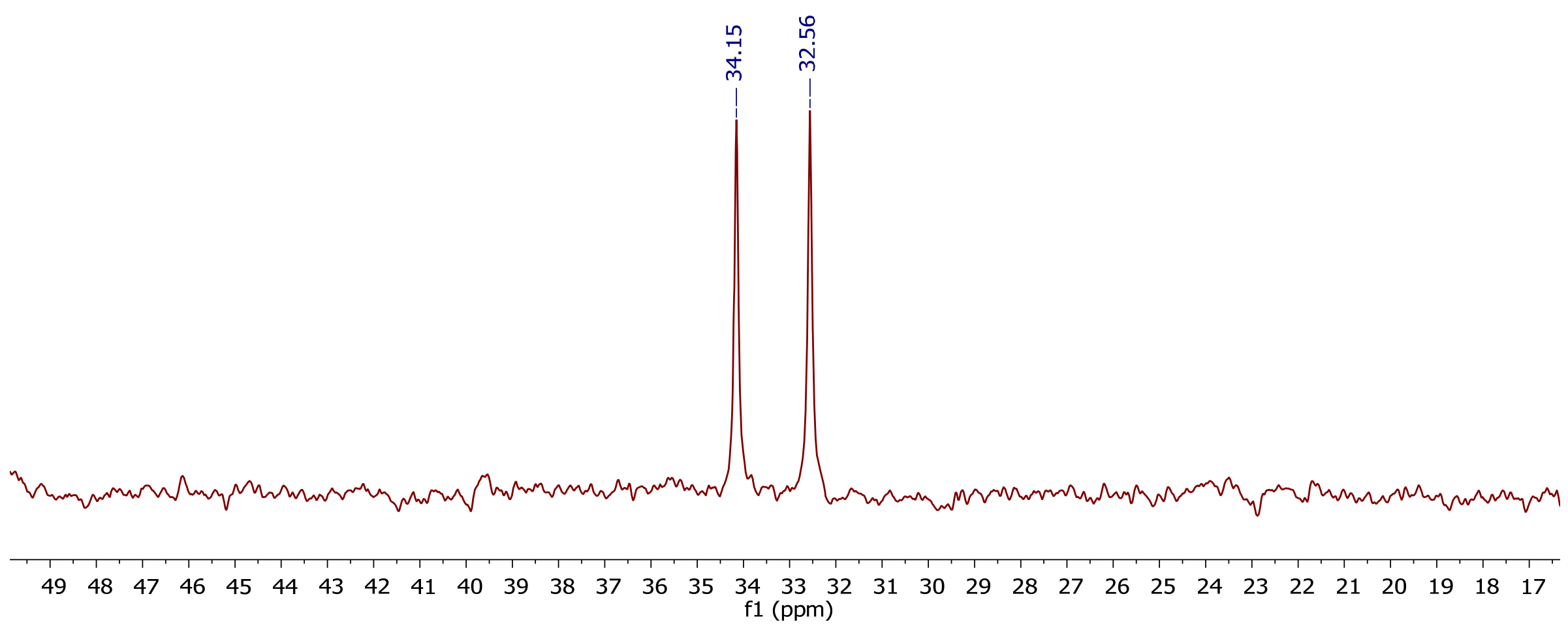

Figure S11. ${ }^{31} \mathrm{P}\left\{{ }^{1} \mathrm{H}\right\}$ NMR spectrum $\left(121.49 \mathrm{MHz}, \mathrm{C}_{6} \mathrm{D}_{6}, 298 \mathrm{~K}\right)$ of $\mathrm{Rh}\{(E)-\mathrm{C}(\mathrm{Et})=\mathrm{C}(\mathrm{Et}) \mathrm{Bpin}\}\left\{\kappa^{3}-\mathrm{POP}-\left[\mathrm{xant}\left(\mathrm{P}^{\mathrm{i}} \mathrm{Pr}_{2}\right)_{2}\right]\right\}(3)$. 


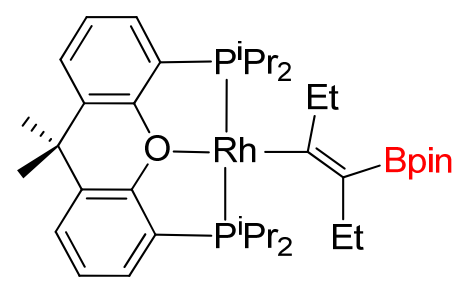

3
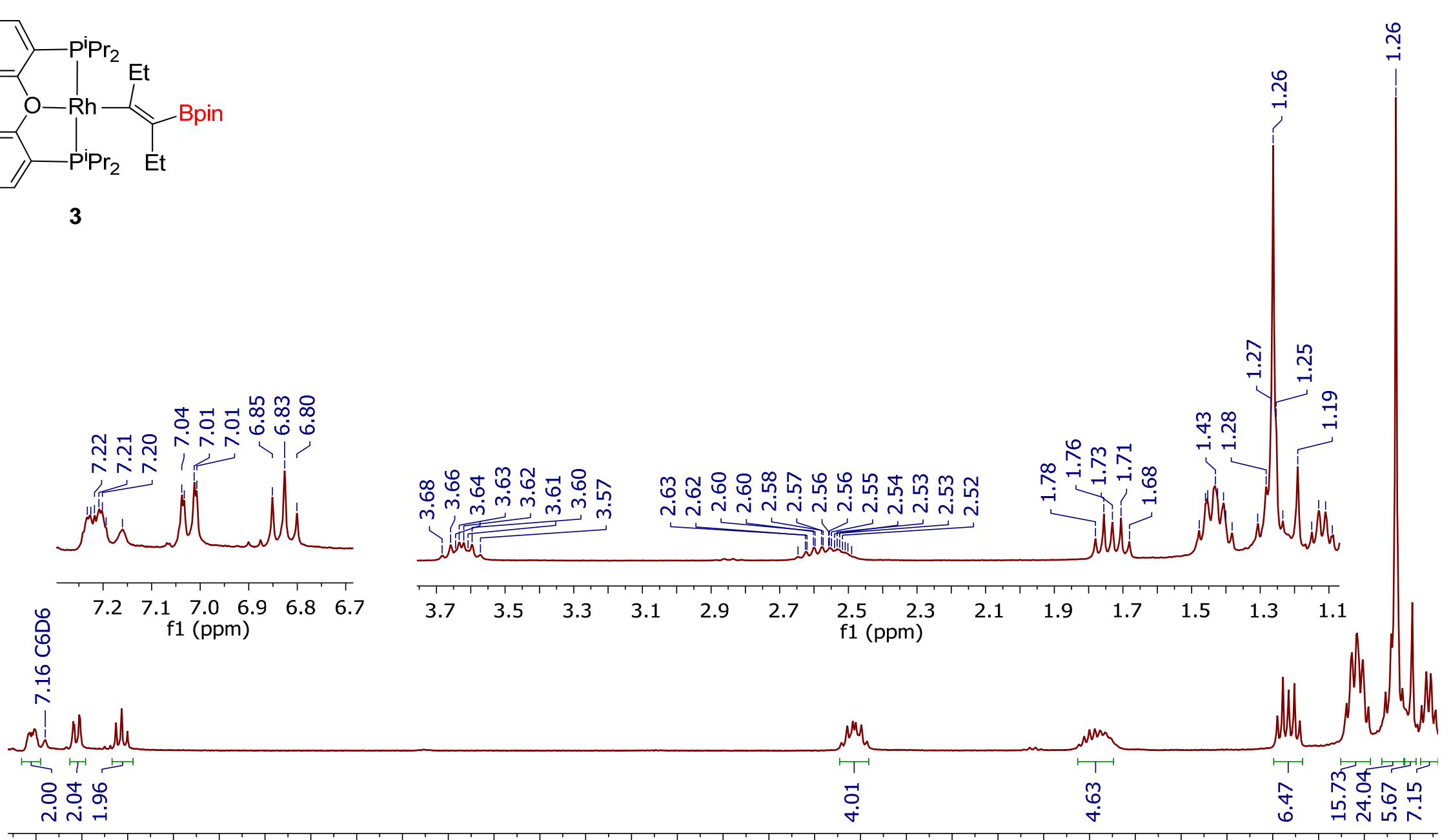

$\begin{array}{lllllllllllllllllllllllllllllllllllllllllllllll}7.2 & 7.0 & 6.8 & 6.6 & 6.4 & 6.2 & 6.0 & 5.8 & 5.6 & 5.4 & 5.2 & 5.0 & 4.8 & 4.6 & 4.4 & 4.2 & 4.0 & 3.8 & 3.6 & 3.4 & 3.2 & 3.0 & 2.8 & 2.6 & 2.4 & 2.2 & 2.0 & 1.8 & 1.6 & 1.4 & 1.2\end{array}$

Figure S12. ${ }^{1} \mathrm{H}$ NMR spectrum $\left(300.13 \mathrm{MHz}, \mathrm{C}_{6} \mathrm{D}_{6}, 298 \mathrm{~K}\right)$ of $\mathrm{Rh}\{(E)-\mathrm{C}(\mathrm{Et})=\mathrm{C}(\mathrm{Et}) \mathrm{Bpin}\}\left\{\kappa^{3}-\mathrm{POP}-\left[\operatorname{xant}\left(\mathrm{P}^{\mathrm{i}} \mathrm{Pr}_{2}\right)_{2}\right]\right\}$ (3). 

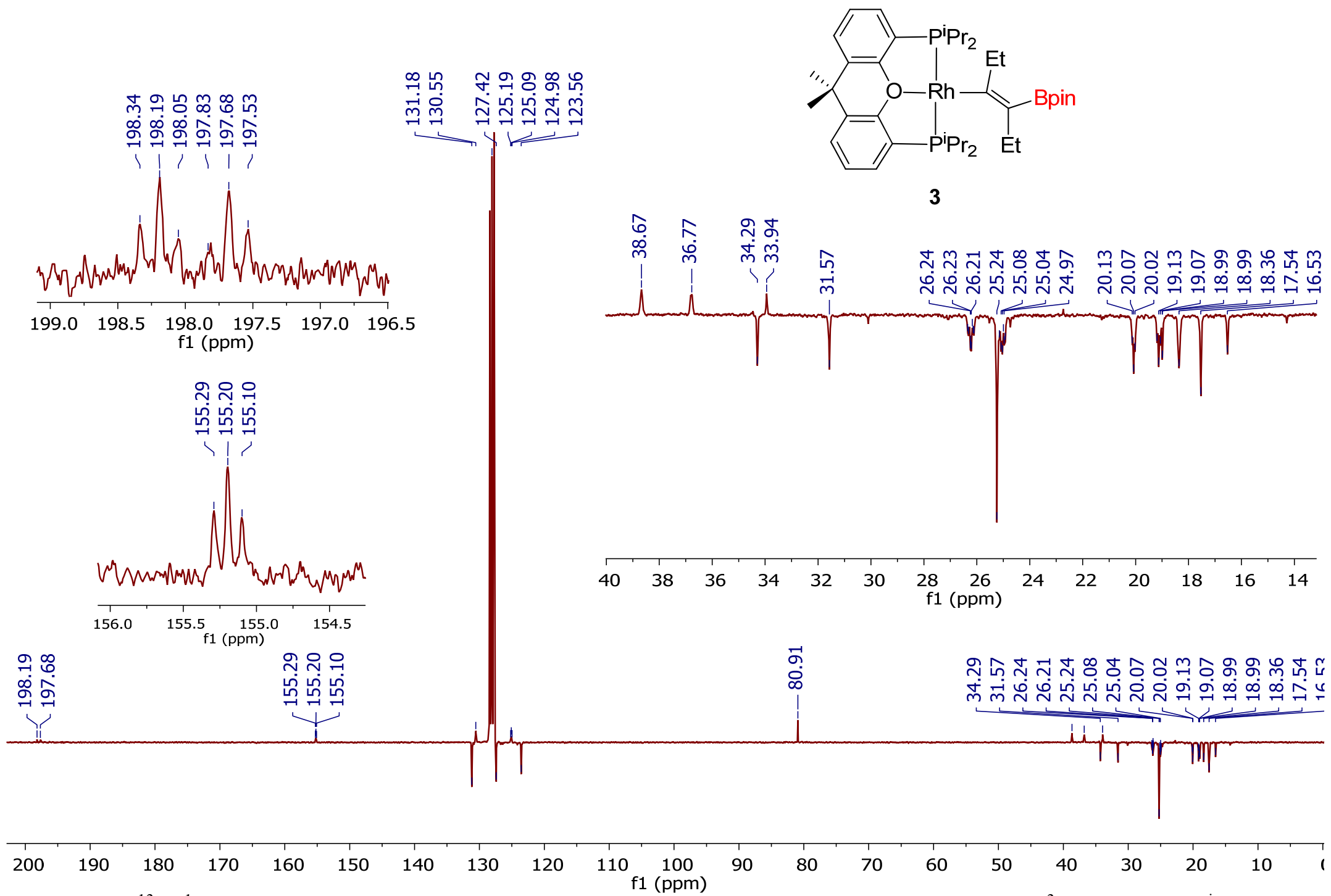

Figure S13. ${ }^{13} \mathrm{C}\left\{{ }^{1} \mathrm{H}\right\}$-apt NMR spectrum $\left(75.48 \mathrm{MHz}, \mathrm{C}_{6} \mathrm{D}_{6}, 298 \mathrm{~K}\right)$ of $\mathrm{Rh}\{(E)-\mathrm{C}(\mathrm{Et})=\mathrm{C}(\mathrm{Et}) \mathrm{Bpin}\}\left\{\kappa^{3}-\mathrm{P}, \mathrm{O}, \mathrm{P}-\left[\operatorname{xant}\left(\mathrm{P}^{\mathrm{i}} \mathrm{Pr}_{2}\right)_{2}\right]\right\}(3)$. 
${ }^{31} \mathrm{P}\left\{{ }^{1} \mathrm{H}\right\} \mathrm{NMR}$ at $298 \mathrm{~K}$
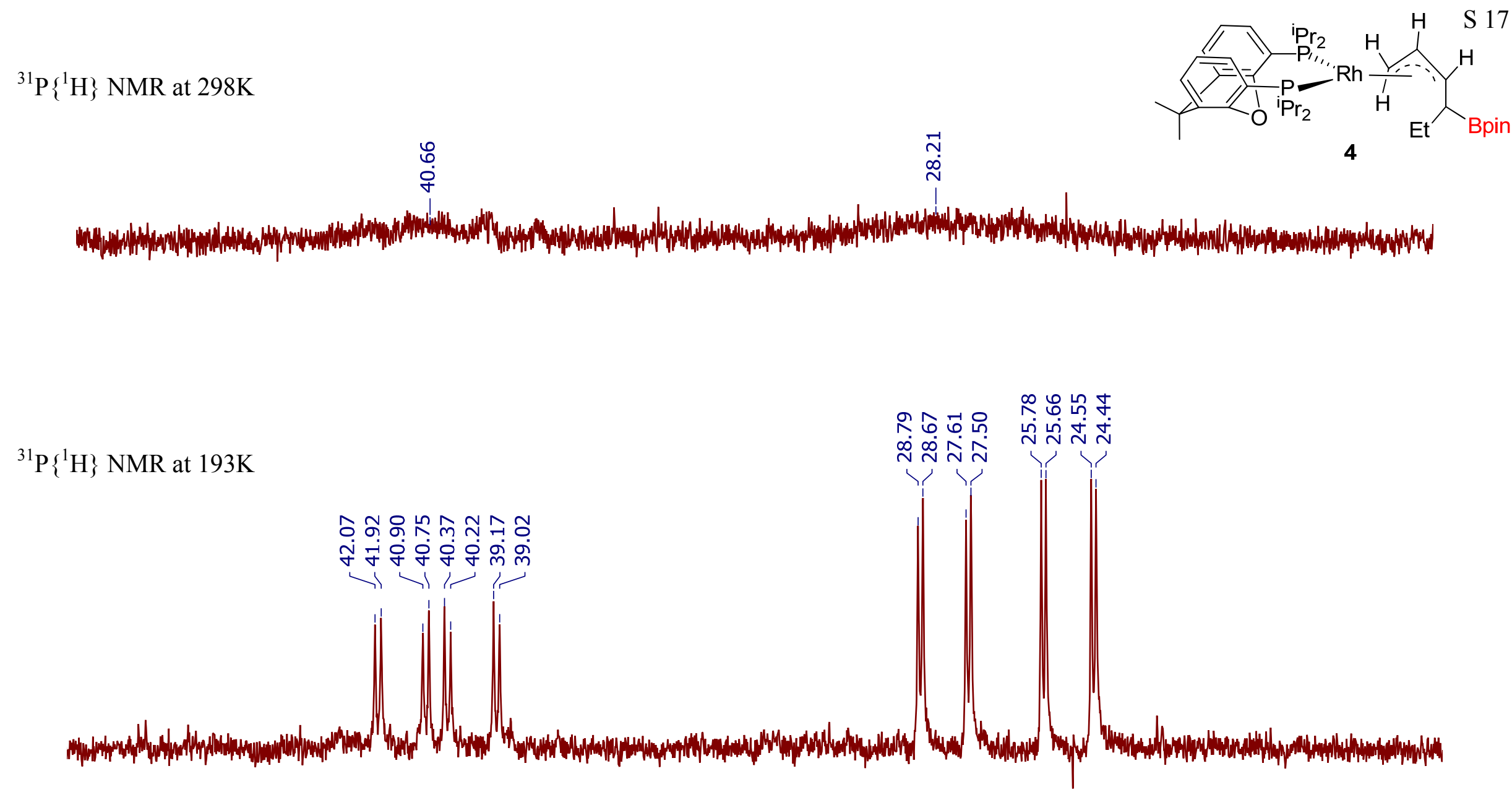

4948474645444342414039383736353433323130292827262524232221201918171 f1 (ppm)

Figure S14. ${ }^{31} \mathrm{P}\left\{{ }^{1} \mathrm{H}\right\}$ NMR spectra (121.49 MHz, $\left.\mathrm{C}_{7} \mathrm{D}_{8}\right)$ of $\mathrm{Rh}\left\{\eta^{3}-\mathrm{CH}_{2} \mathrm{CHCHCH}(\mathrm{Bpin}) \mathrm{Et}\right\}\left\{\kappa^{2}-\mathrm{P}, \mathrm{P}-\left[\mathrm{xant}\left(\mathrm{P}^{\mathrm{i}} \mathrm{Pr}_{2}\right)_{2}\right]\right\}$ (4) at 298 and $193 \mathrm{~K}$. 


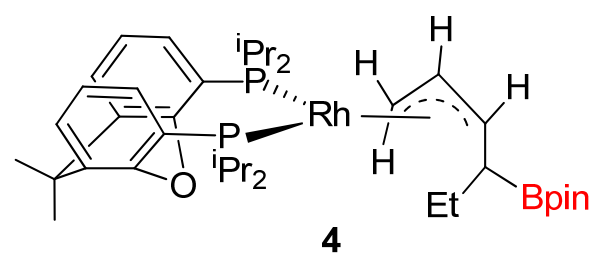

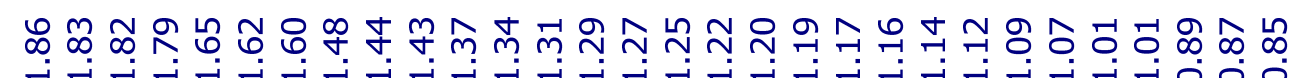

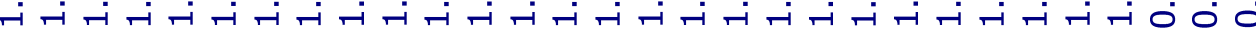

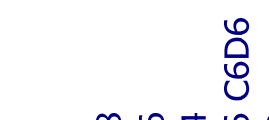

乌ి

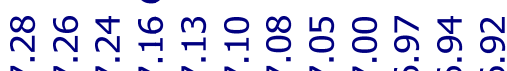

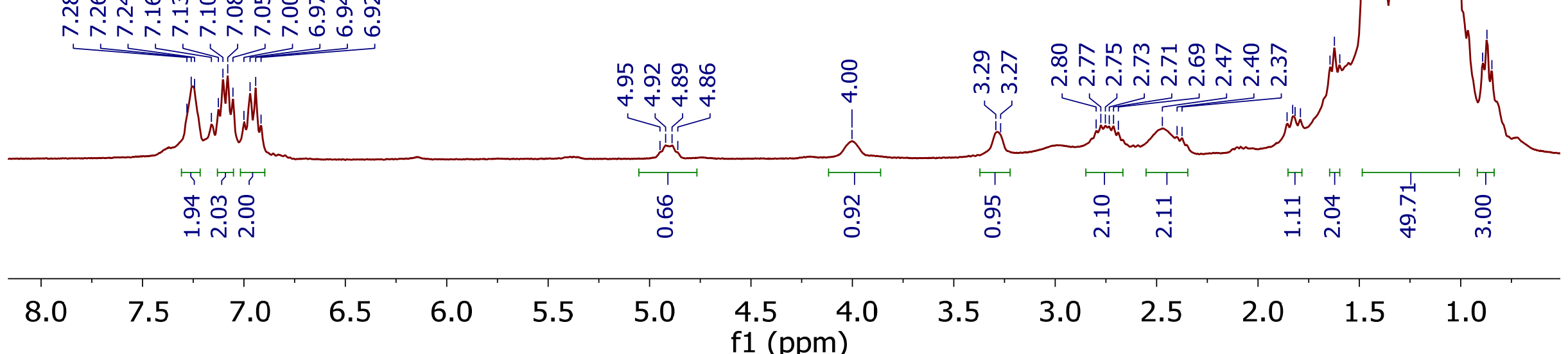

Figure S15. ${ }^{1} \mathrm{H}$ NMR spectrum $\left(300.13 \mathrm{MHz}, \mathrm{C}_{6} \mathrm{D}_{6}, 298 \mathrm{~K}\right)$ of $\mathrm{Rh}\left\{\eta^{3}-\mathrm{CH}_{2} \mathrm{CHCHCH}(\mathrm{Bpin}) \mathrm{Et}\right\}\left\{\kappa^{2}-\mathrm{P}, \mathrm{P}-\left[\operatorname{xant}\left(\mathrm{P}^{\mathrm{i}} \mathrm{Pr}_{2}\right)_{2}\right]\right\}(4)$. 


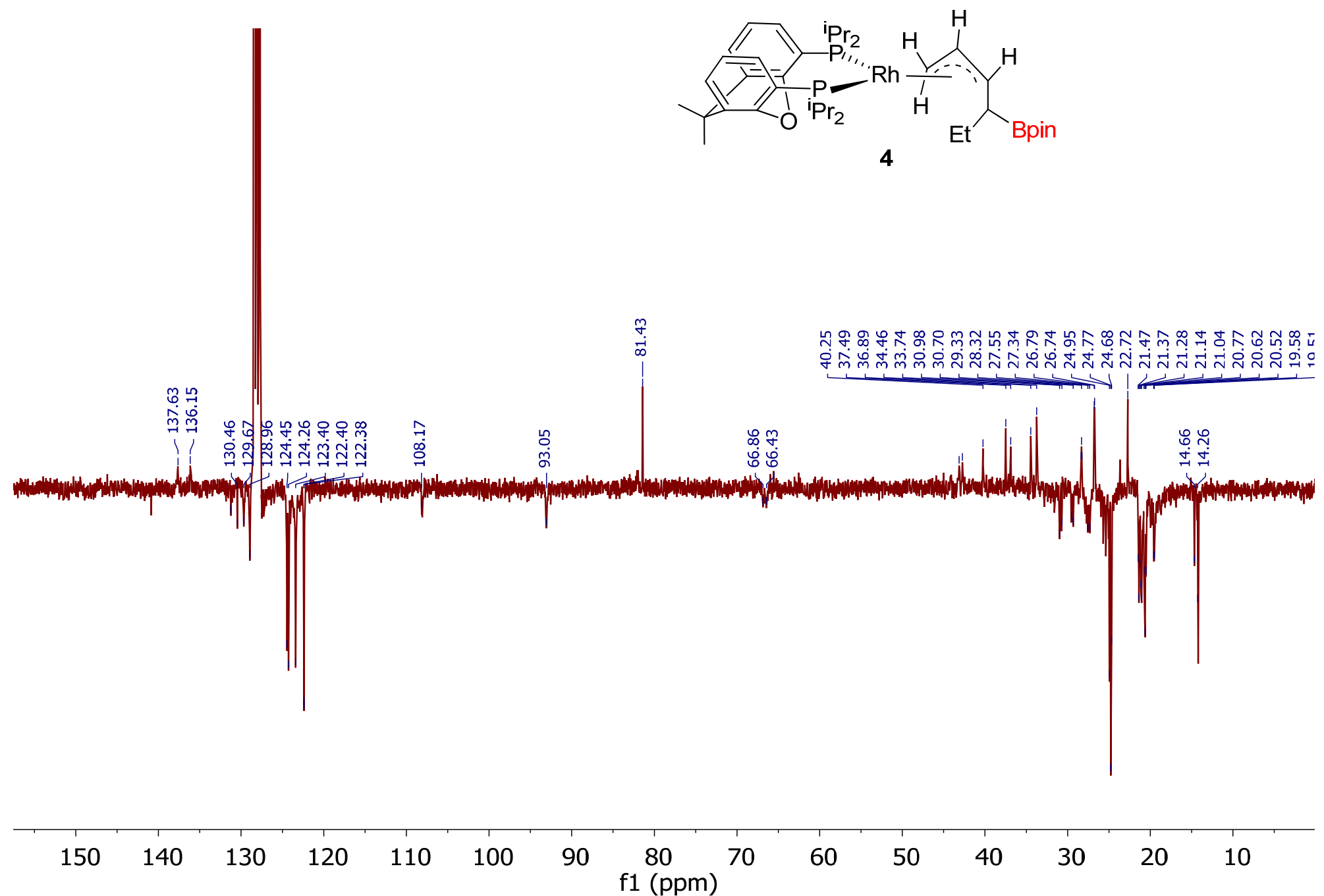

Figure S16. ${ }^{13} \mathrm{C}\left\{{ }^{1} \mathrm{H}\right\}$-apt NMR spectrum $\left(75.48 \mathrm{MHz}, \mathrm{C}_{6} \mathrm{D}_{6}, 298 \mathrm{~K}\right)$ of $\mathrm{Rh}\left\{\eta^{3}-\mathrm{CH}_{2} \mathrm{CHCHCH}(\mathrm{Bpin}) \mathrm{Et}\right\}\left\{\kappa^{2}-\mathrm{P}, \mathrm{P}-\left[\operatorname{xant}\left(\mathrm{P}^{\mathrm{i}} \mathrm{Pr}_{2}\right)_{2}\right]\right\}(4)$. 


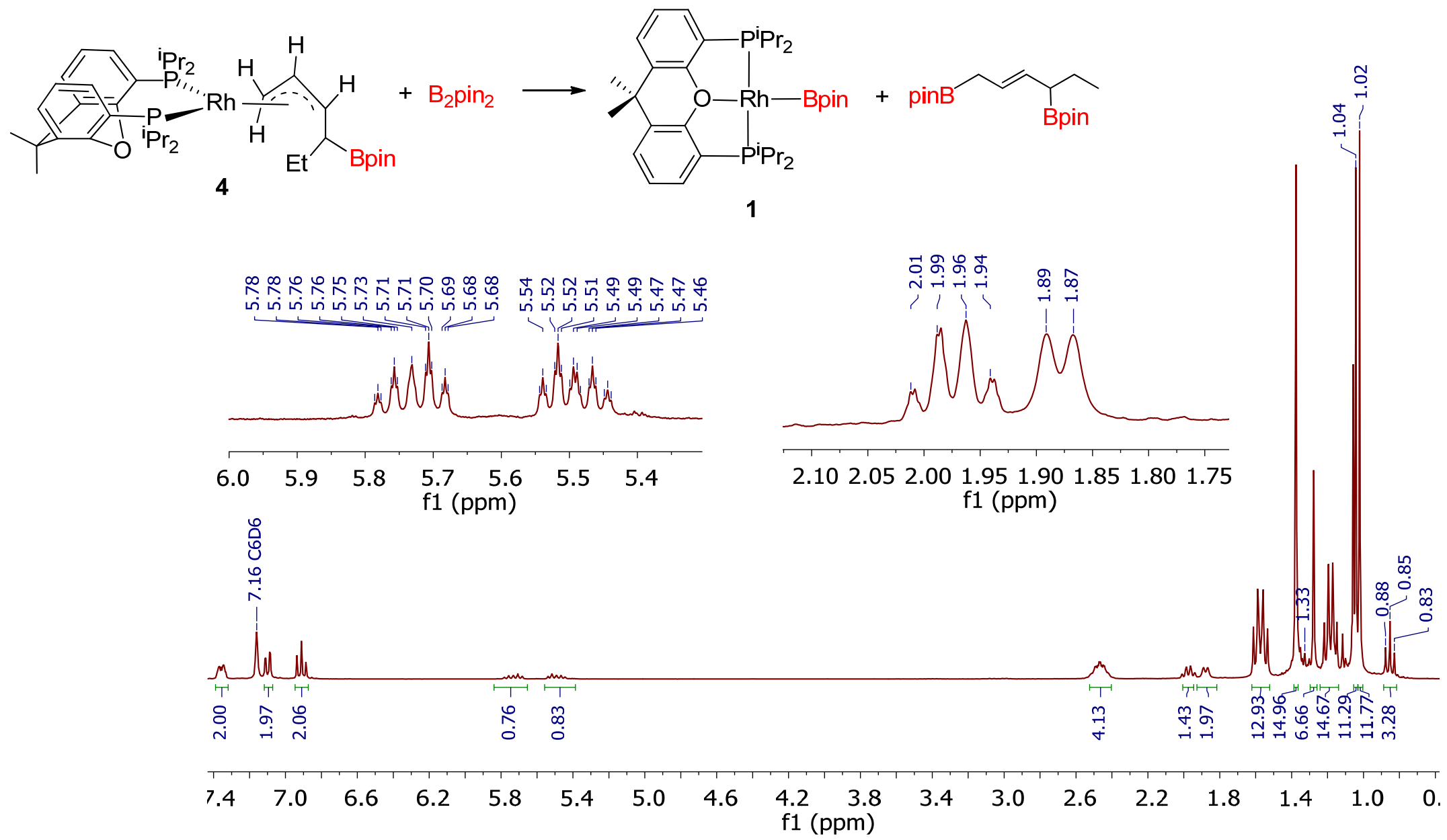

Figure S17. ${ }^{1} \mathrm{H}$ NMR spectrum $\left(300.13 \mathrm{MHz}, \mathrm{C}_{6} \mathrm{D}_{6}, 298 \mathrm{~K}\right)$ of the reaction of $\mathrm{Rh}\left\{\eta^{3}-\mathrm{CH}_{2} \mathrm{CHCHCH}(\mathrm{Bpin}) \mathrm{Et}\right\}\left\{\kappa^{2}-\mathrm{P}, \mathrm{P}-\left[\mathrm{xant}\left(\mathrm{P}^{\mathrm{i}} \mathrm{Pr}_{2}\right)_{2}\right]\right\}(4)$ with $\mathrm{B}_{2}$ pin $_{2}$ to give complex 1 and 1,4-dipinacolboryl- $(E)$-2-hexene. 

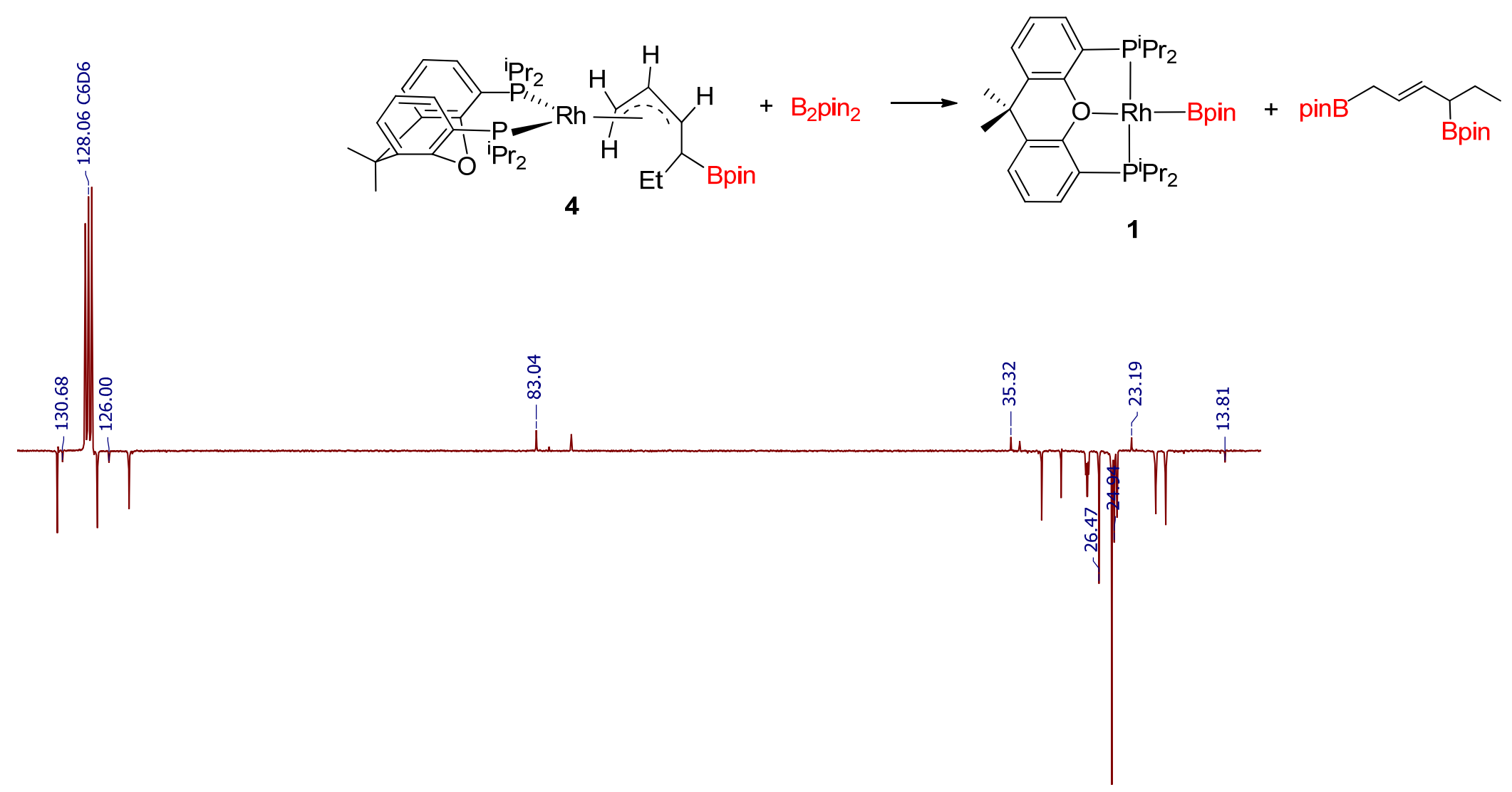

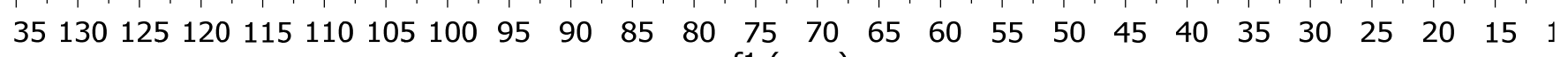

Figure S18. ${ }^{13} \mathrm{C}\left\{{ }^{1} \mathrm{H}\right\}$ NMR spectrum $\left(75.48 \mathrm{MHz}, \mathrm{C}_{6} \mathrm{D}_{6}, 298 \mathrm{~K}\right)$ of the reaction of $\mathrm{Rh}\left\{\eta^{3}-\mathrm{CH}_{2} \mathrm{CHCHCH}(\mathrm{Bpin}) \mathrm{Et}\right\}\left\{\kappa^{2}-\mathrm{P}, \mathrm{P}-\left[\mathrm{xant}\left(\mathrm{P}^{\mathrm{i}} \mathrm{Pr}\right)_{2}\right]\right\}(4)$ with $\mathrm{B}_{2} \operatorname{pin}_{2}$ to give complex 1 and 1,4-dipinacolboryl-(E)-2-hexene. 


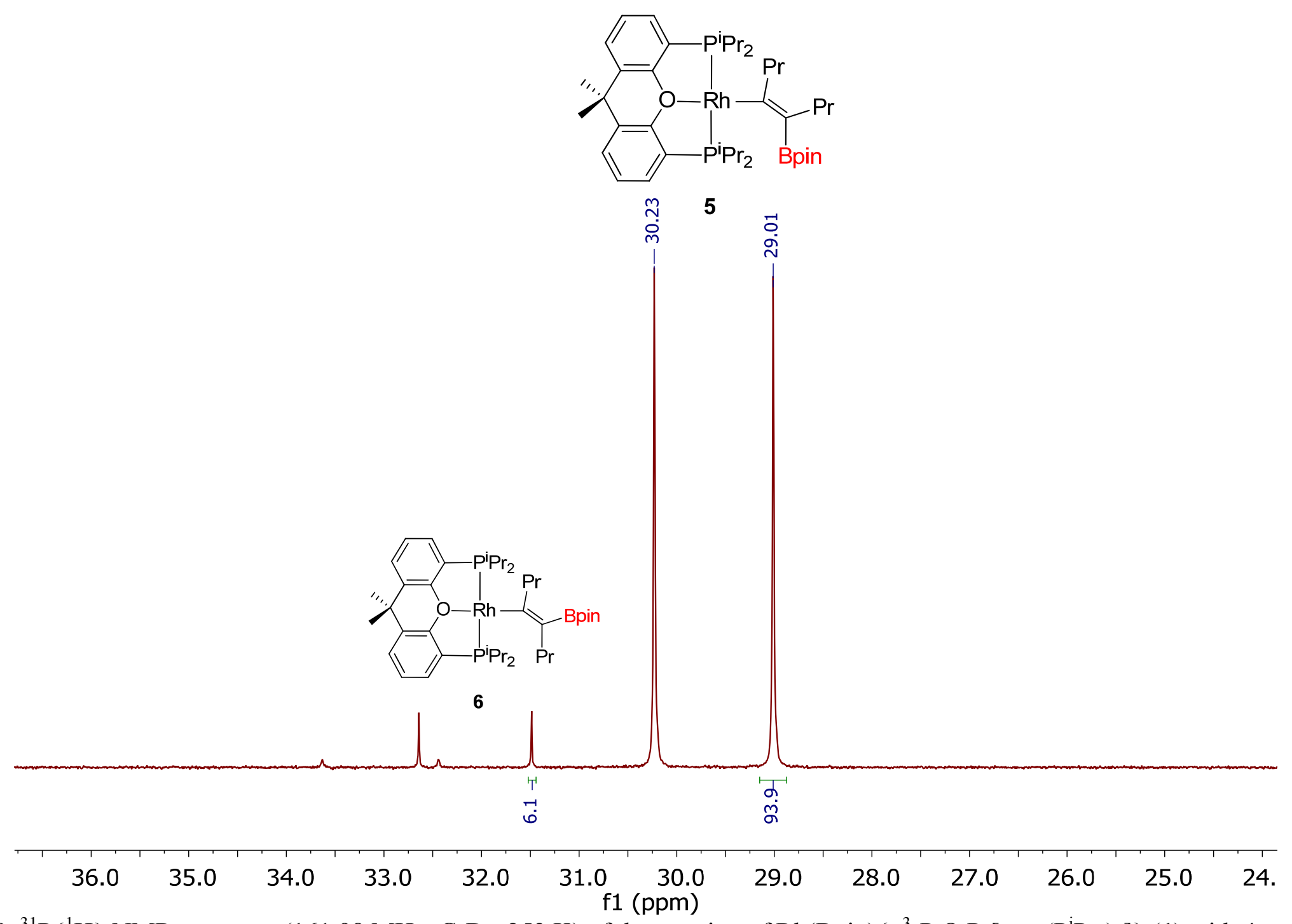

Figure S19. ${ }^{31} \mathrm{P}\left\{{ }^{1} \mathrm{H}\right\}$ NMR spectrum $\left(161.98 \mathrm{MHz}, \mathrm{C}_{7} \mathrm{D}_{8}, 253 \mathrm{~K}\right)$ of the reaction of $\mathrm{Rh}(\mathrm{Bpin})\left\{\kappa^{3}-\mathrm{P}, \mathrm{O}, \mathrm{P}-\left[\operatorname{xant}\left(\mathrm{P}^{\mathrm{i}} \mathrm{Pr}_{2}\right)_{2}\right]\right\}(\mathbf{1})$ with 4-octyne at low temperature: Spectroscopic detection of $\operatorname{Rh}\{(Z)-C(P r)=C(\operatorname{Pr}) \operatorname{Bpin}\}\left\{\kappa^{3}-\mathrm{POP}-\left[\operatorname{xant}\left(\mathrm{P}^{\mathrm{i}} \mathrm{Pr}_{2}\right)_{2}\right]\right\}$ (5). 

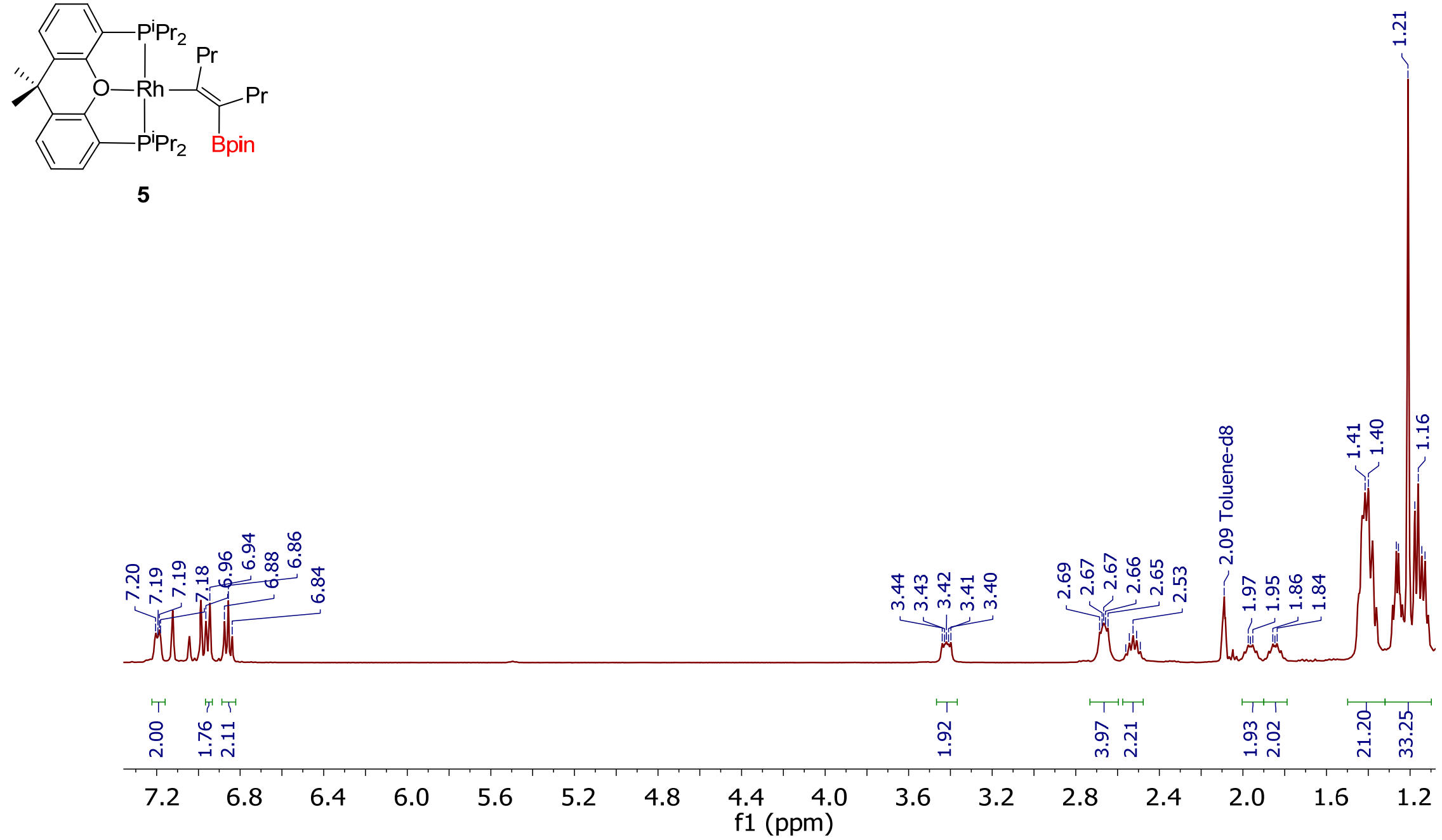

Figure S20. ${ }^{1} \mathrm{H}$ NMR spectrum $\left(400.13 \mathrm{MHz}, \mathrm{C}_{7} \mathrm{D}_{8}, 253 \mathrm{~K}\right)$ of the reaction of $\mathrm{Rh}(\mathrm{Bpin})\left\{\kappa^{3}-\mathrm{P}, \mathrm{O}, \mathrm{P}-\left[\mathrm{xant}\left(\mathrm{P}^{\mathrm{i}} \mathrm{Pr}_{2}\right)_{2}\right]\right\}(\mathbf{1})$ with $4-\mathrm{octyne}$ at low temperature: Spectroscopic detection of $\operatorname{Rh}\{(Z)-\mathrm{C}(\mathrm{Pr})=\mathrm{C}(\mathrm{Pr}) \mathrm{Bpin}\}\left\{\kappa^{3}-\mathrm{POP}-\left[\operatorname{xant}\left(\mathrm{P}^{\mathrm{i}} \mathrm{Pr}_{2}\right)_{2}\right]\right\}$ (5). 

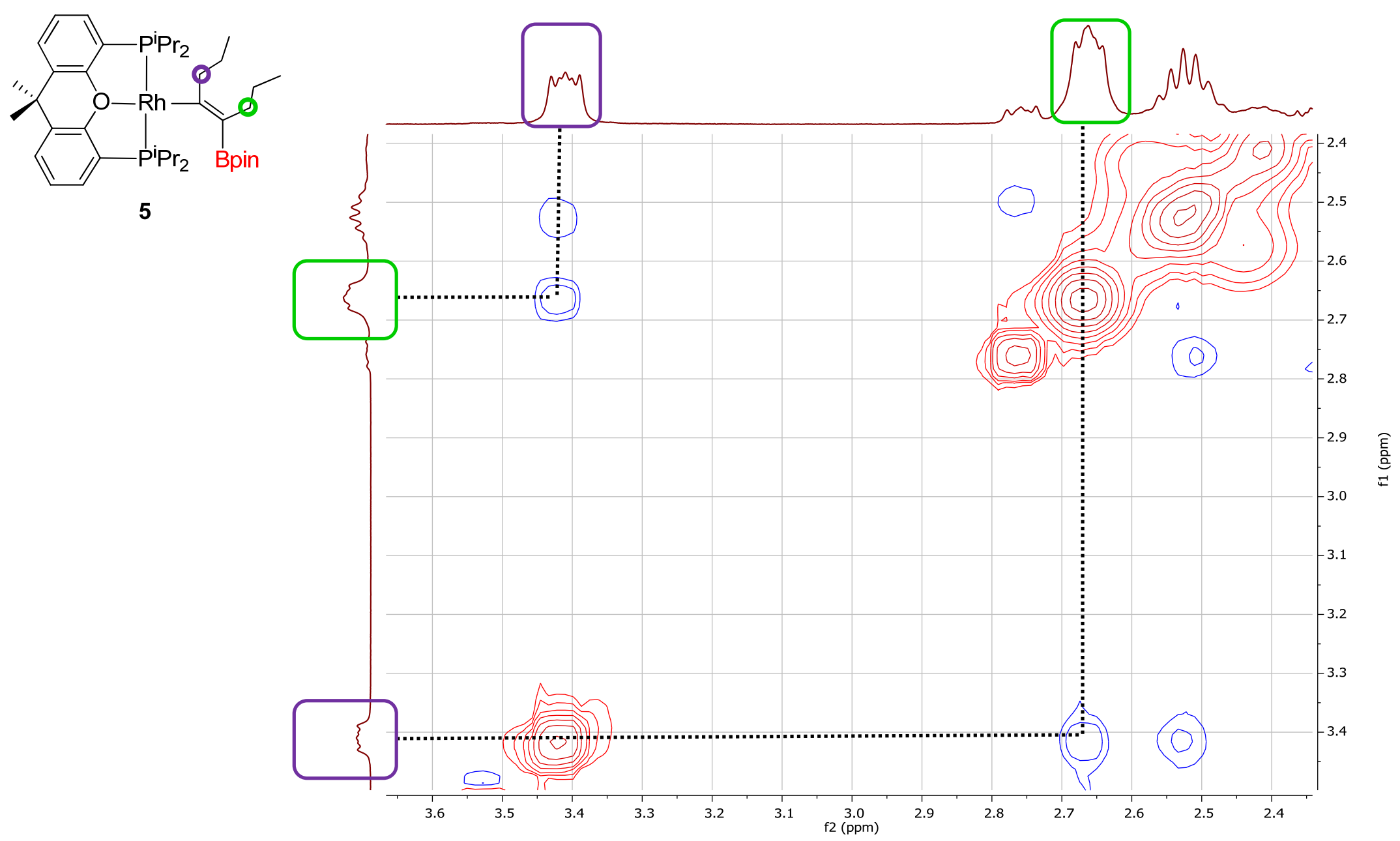

Figure S21. Partial view of the ${ }^{1} \mathrm{H},{ }^{1} \mathrm{H}$ NOESY NMR spectrum $\left(400.13 \mathrm{MHz}, \mathrm{C}_{7} \mathrm{D}_{8}, 253 \mathrm{~K}\right)$ of $\mathrm{Rh}\{(Z)-\mathrm{C}(\operatorname{Pr})=\mathrm{C}(\operatorname{Pr}) \mathrm{Bpin}\}\left\{\kappa^{3}-\mathrm{P}, \mathrm{O}, \mathrm{P}-\right.$ [xant $\left.\left.\left(\mathrm{P}^{\mathrm{i}} \mathrm{Pr}_{2}\right)_{2}\right]\right\}$ (5). 


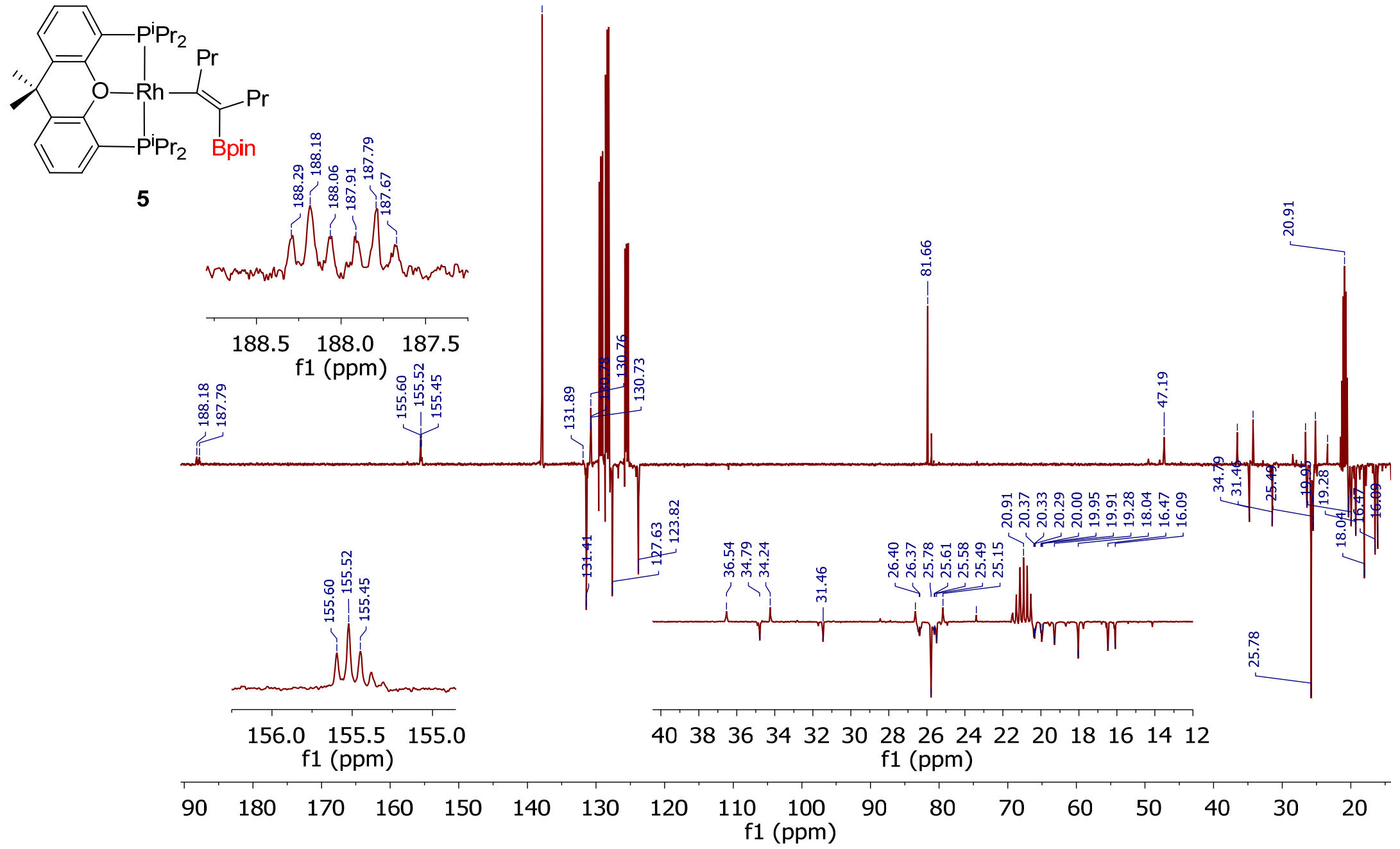

Figure S22. ${ }^{13} \mathrm{C}\left\{{ }^{1} \mathrm{H}\right\}$-apt NMR spectrum $\left(100.62 \mathrm{MHz}, \mathrm{C}_{7} \mathrm{D}_{8}, 253 \mathrm{~K}\right)$ of the reaction of $\mathrm{Rh}(\mathrm{Bpin})\left\{\kappa^{3}-\mathrm{P}, \mathrm{O}, \mathrm{P}-\left[\mathrm{xant}\left(\mathrm{P}^{\mathrm{i}} \mathrm{Pr}_{2}\right)_{2}\right]\right\}(\mathbf{1})$ with $4-\mathrm{octyne}$ at low temperature: Spectroscopic detection of $\operatorname{Rh}\{(Z)-C(\operatorname{Pr})=C(\operatorname{Pr}) \operatorname{Bpin}\}\left\{\kappa^{3}-\mathrm{POP}-\left[\operatorname{xant}\left(\mathrm{P}^{\mathrm{i}} \mathrm{Pr}_{2}\right)_{2}\right]\right\}(5)$. 

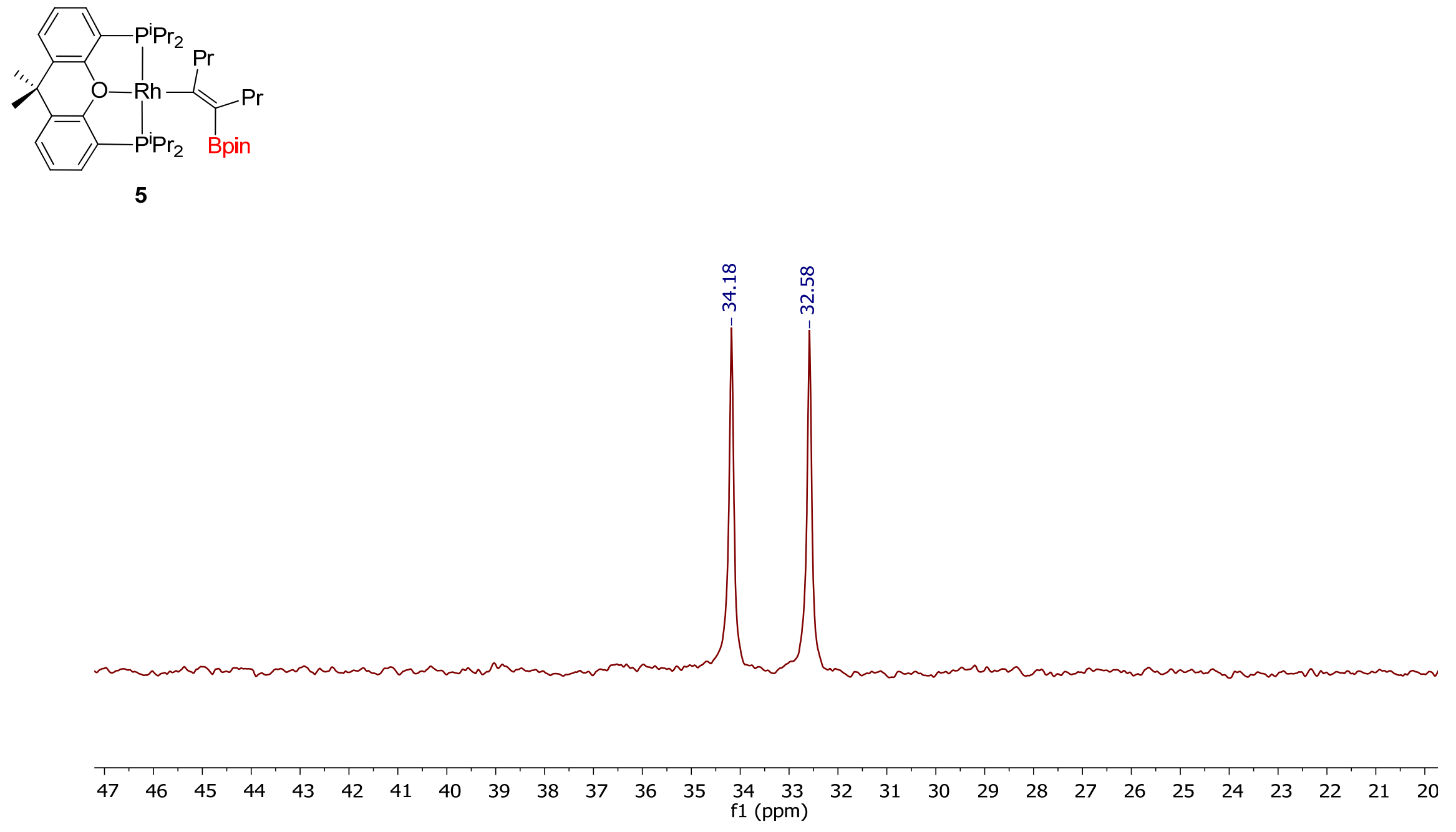

Figure S23. ${ }^{31} \mathrm{P}\left\{{ }^{1} \mathrm{H}\right\}$ NMR spectrum $\left(121.49 \mathrm{MHz}, \mathrm{C}_{6} \mathrm{D}_{6}, 298 \mathrm{~K}\right)$ of $\mathrm{Rh}\{(E)-\mathrm{C}(\mathrm{Pr})=\mathrm{C}(\operatorname{Pr}) \mathrm{Bpin}\}\left\{\kappa^{3}-\mathrm{POP}-\left[\operatorname{xant}\left(\mathrm{P}^{\mathrm{i}} \mathrm{Pr}_{2}\right)_{2}\right]\right\}(6)$ 


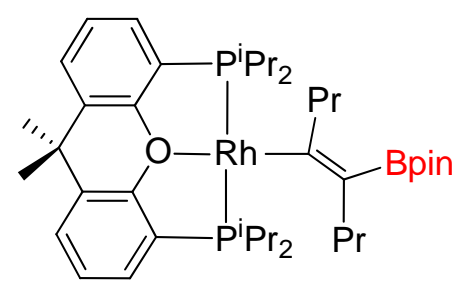

6

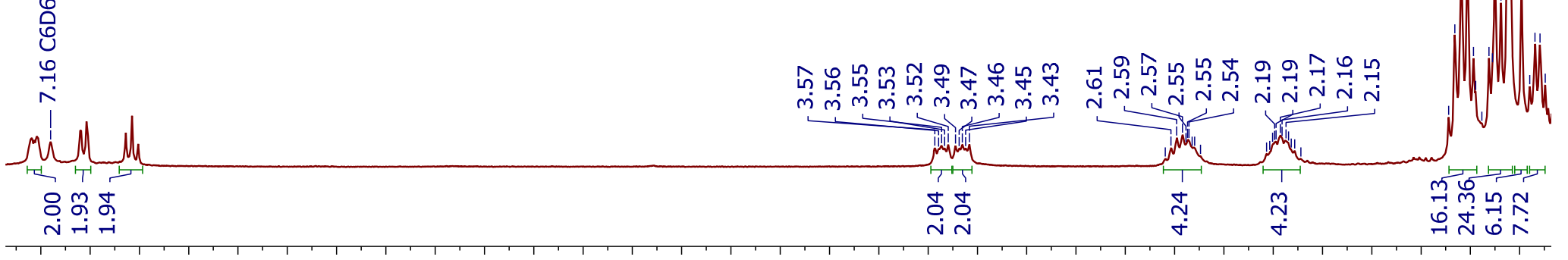

$\begin{array}{lllllllllllllllllllllllllllllllllllllll}7.2 & 7.0 & 6.8 & 6.6 & 6.4 & 6.2 & 6.0 & 5.8 & 5.6 & 5.4 & 5.2 & 5.0 & 4.8 & 4.6 & 4.4 & 4.2 & 4.0 & 3.8 & 3.6 & 3.4 & 3.2 & 3.0 & 2.8 & 2.6 & 2.4 & 2.2 & 2.0 & 1.8 & 1.6 & 1.4 & 1.2\end{array}$

Figure S24. ${ }^{1} \mathrm{H}$ NMR spectrum $\left(300.13 \mathrm{MHz}, \mathrm{C}_{6} \mathrm{D}_{6}, 298 \mathrm{~K}\right)$ of $\mathrm{Rh}\{(E)-\mathrm{C}(\mathrm{Pr})=\mathrm{C}(\operatorname{Pr}) \operatorname{Bpin}\}\left\{\kappa^{3}-\mathrm{POP}-\left[\operatorname{xant}\left(\mathrm{P}^{\mathrm{i}} \mathrm{Pr}_{2}\right)_{2}\right]\right\}(6)$. 

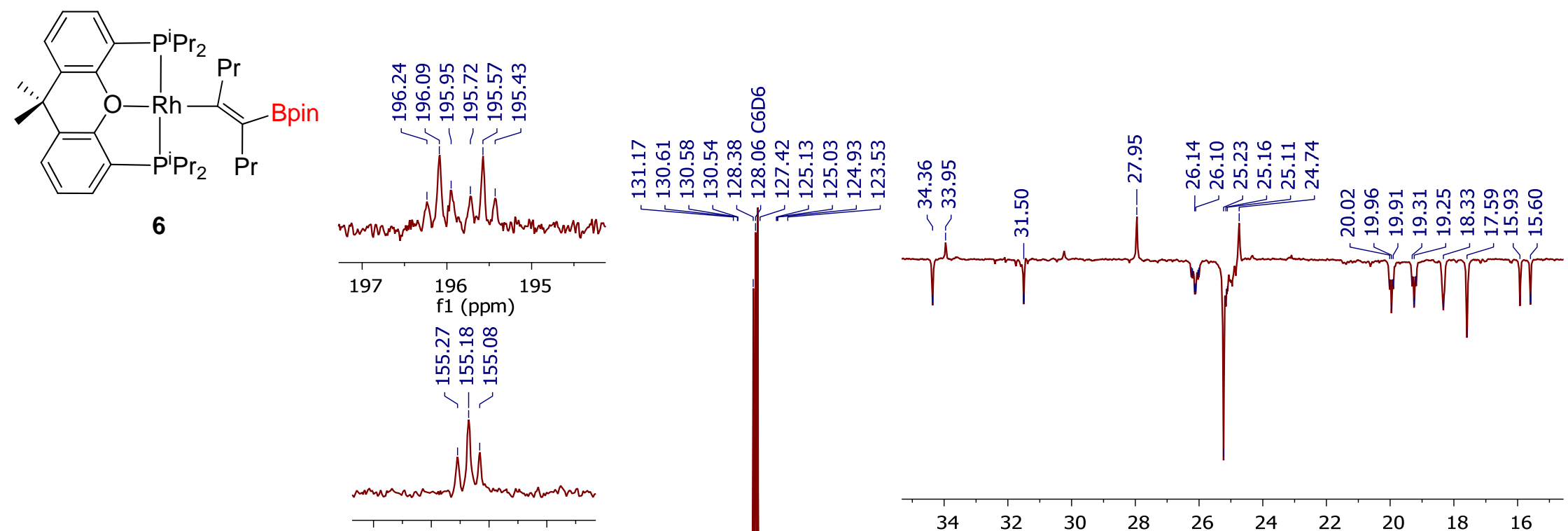

156.0155 .5155 .0154 .5 f1 (ppm)

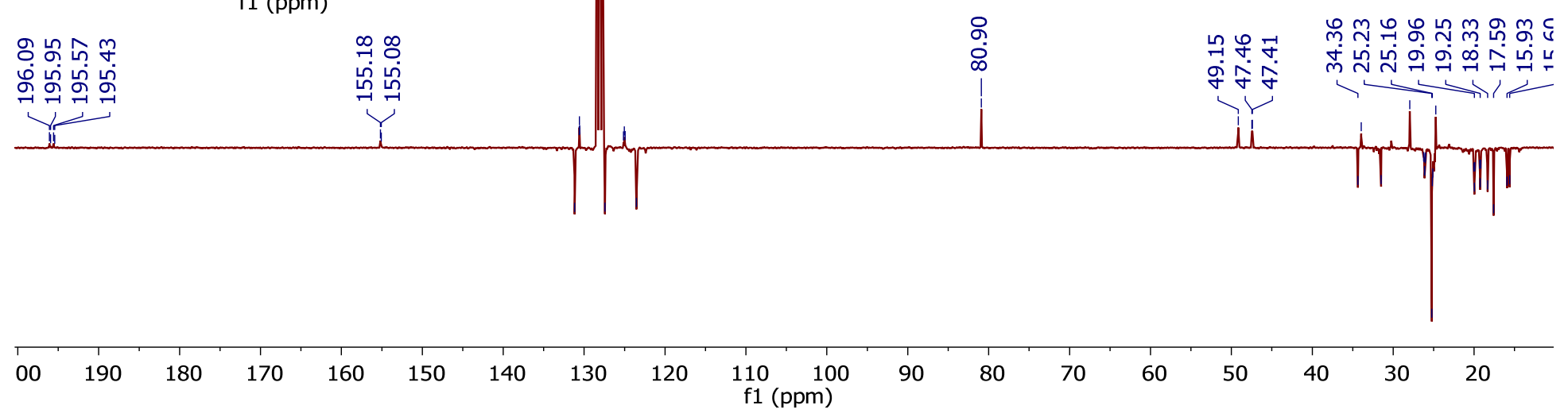

Figure S25. ${ }^{13} \mathrm{C}\left\{{ }^{1} \mathrm{H}\right\}$-apt NMR spectrum $\left(75.48 \mathrm{MHz}, \mathrm{C}_{6} \mathrm{D}_{6}, 298 \mathrm{~K}\right)$ of $\mathrm{Rh}\{(E)-\mathrm{C}(\mathrm{Pr})=\mathrm{C}(\operatorname{Pr}) \mathrm{Bpin}\}\left\{\kappa^{3}-\mathrm{POP}-\left[\mathrm{xant}\left(\mathrm{P}^{\mathrm{i}} \mathrm{Pr}_{2}\right)_{2}\right]\right\}(6)$. 
${ }^{31} \mathrm{P}\left\{{ }^{1} \mathrm{H}\right\} \mathrm{NMR}$ at $298 \mathrm{~K}$
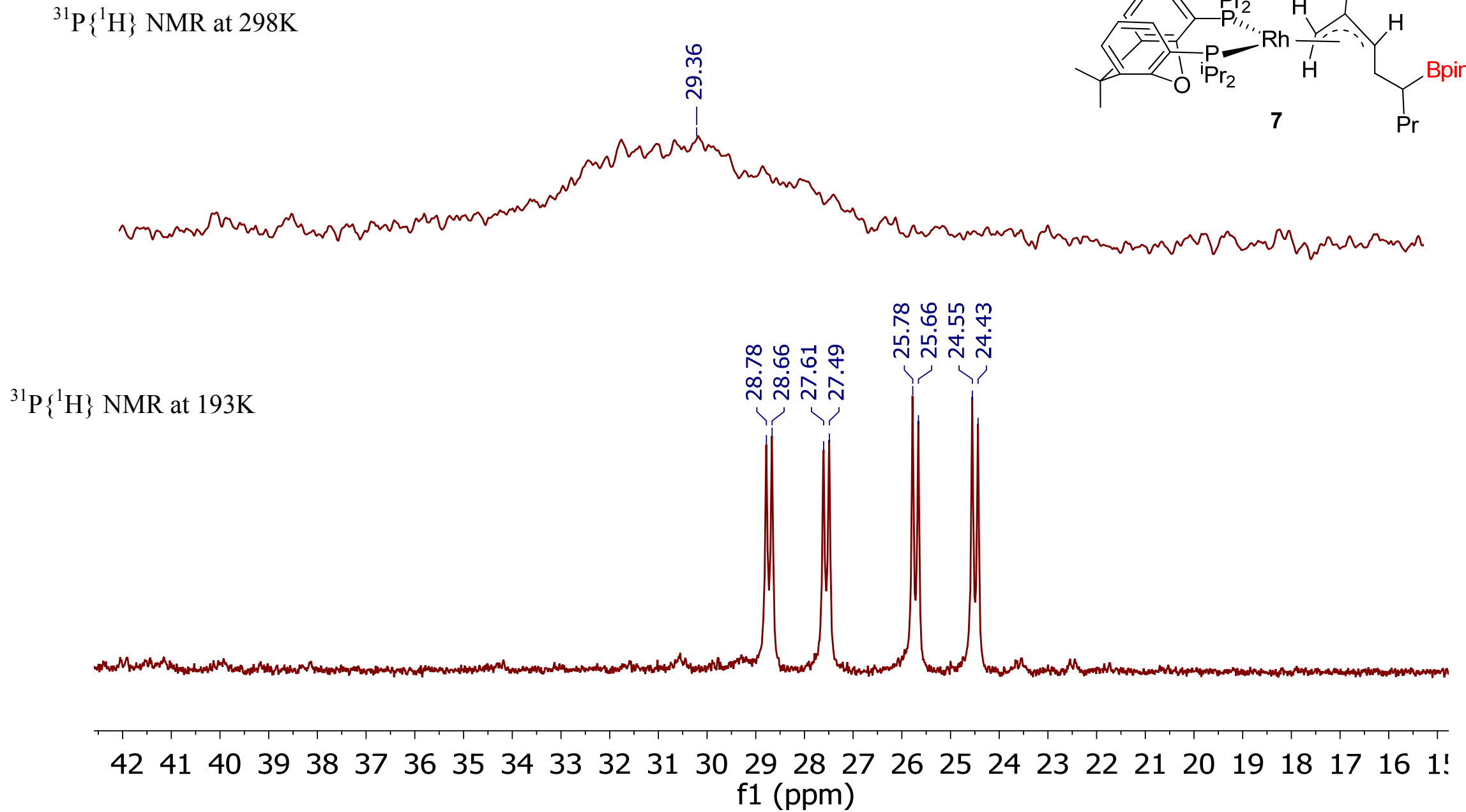

Figure S26. ${ }^{31} \mathrm{P}\left\{{ }^{1} \mathrm{H}\right\}$ NMR spectra $\left(161.98 \mathrm{MHz}\right.$, toluene- $\left.d_{8}\right)$ of $\mathrm{Rh}\left\{\eta^{3}-\mathrm{CH}_{2} \mathrm{CHCHCH}_{2} \mathrm{CH}(\mathrm{Bpin}) \mathrm{Pr}\right\}\left\{\kappa^{2}-\mathrm{P}, \mathrm{P}-\left[\mathrm{xant}\left(\mathrm{P}^{\mathrm{i}} \mathrm{Pr}_{2}\right)_{2}\right]\right\}(7)$ at 298 and $193 \mathrm{~K}$. 

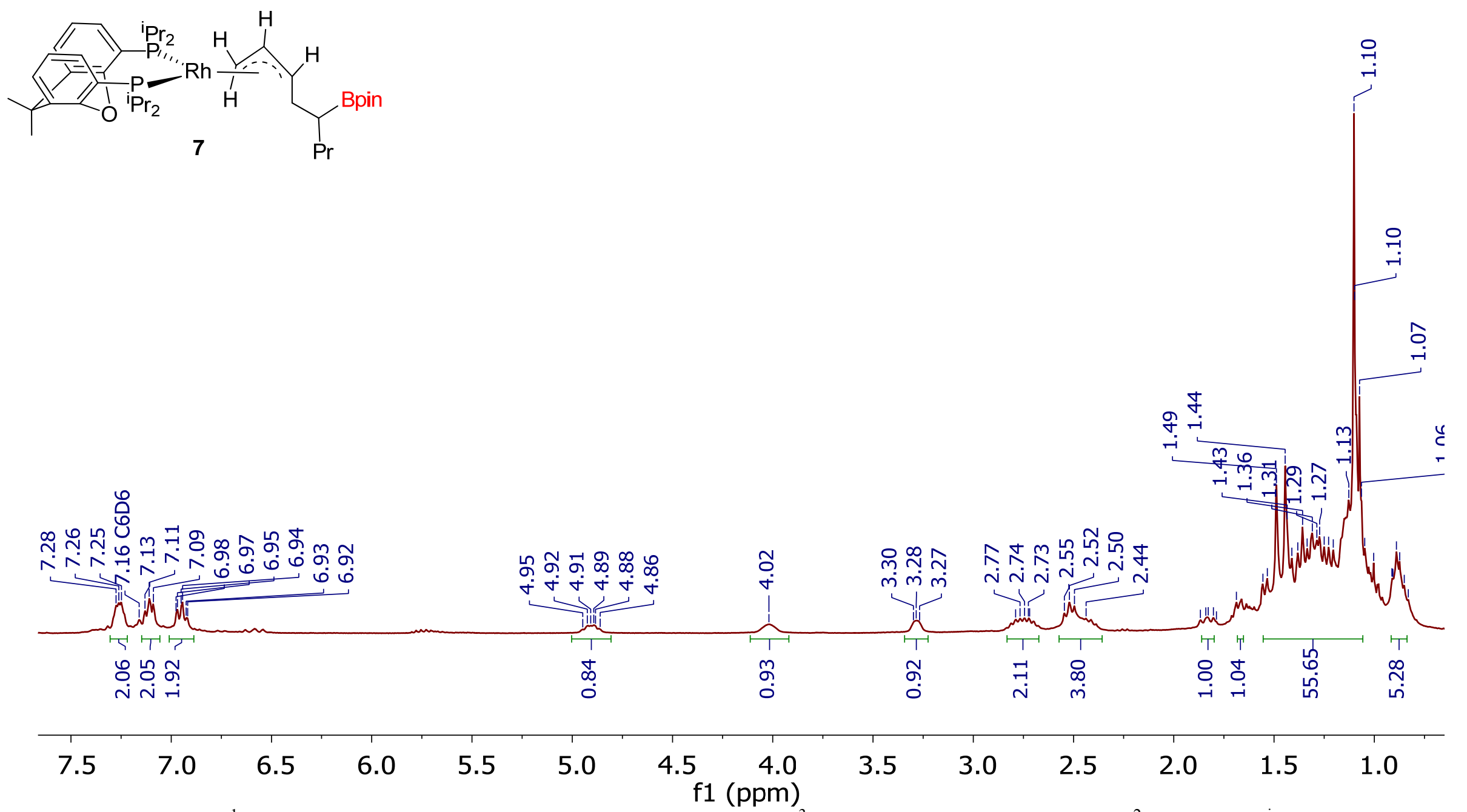

Figure S27. ${ }^{1} \mathrm{H}$ NMR spectrum $\left(300.13 \mathrm{MHz}, \mathrm{C}_{6} \mathrm{D}_{6}, 298 \mathrm{~K}\right)$ of $\mathrm{Rh}\left\{\eta^{3}-\mathrm{CH}_{2} \mathrm{CHCHCH}_{2} \mathrm{CH}(\mathrm{Bpin}) \mathrm{Pr}\right\}\left\{\kappa^{2}-\mathrm{P}, \mathrm{P}-\left[\operatorname{xant}\left(\mathrm{P}^{\mathrm{i}} \mathrm{Pr}_{2}\right)_{2}\right]\right\}(7)$. 


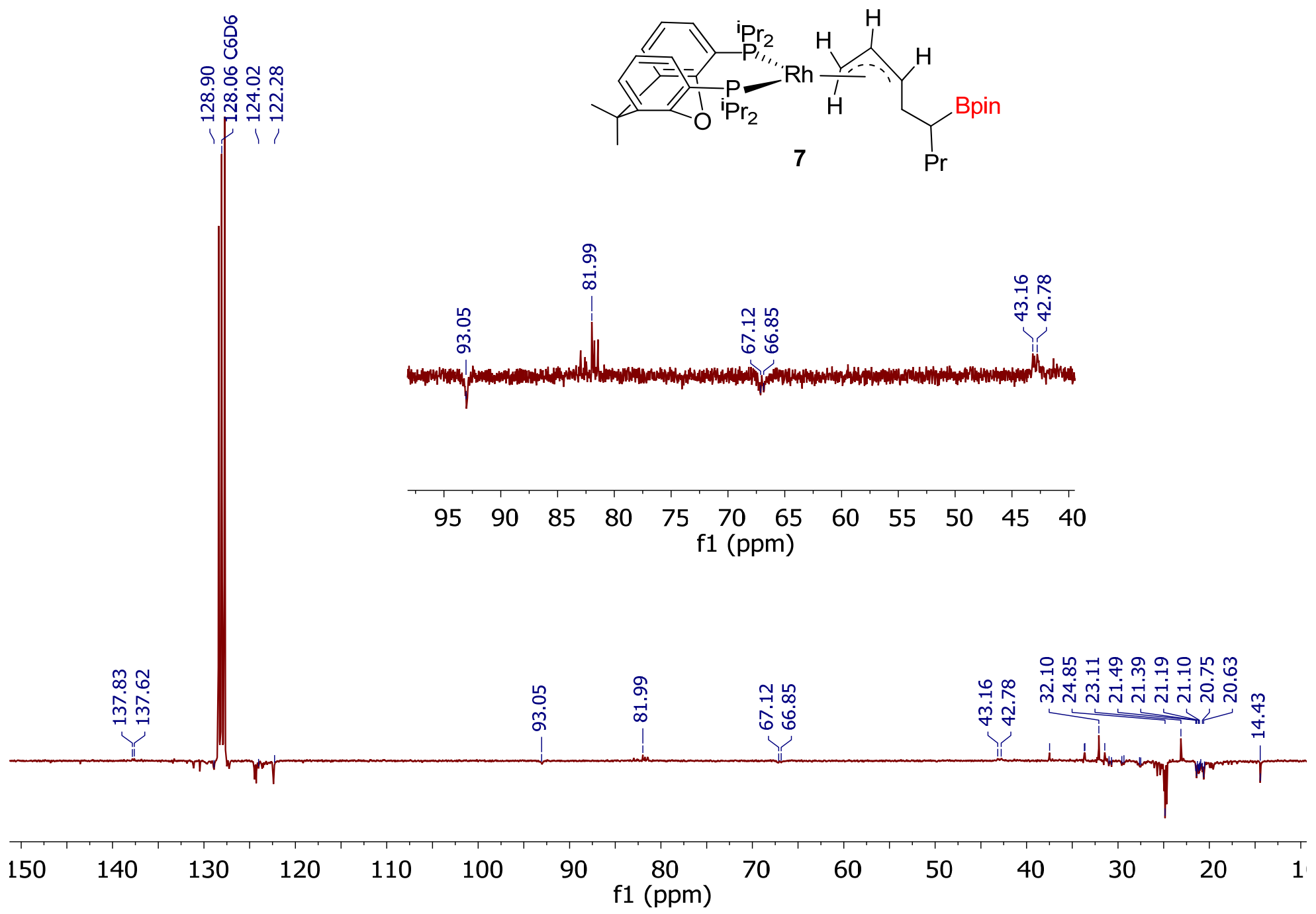

Figure S28. ${ }^{13} \mathrm{C}\left\{{ }^{1} \mathrm{H}\right\}$-apt NMR spectrum $\left(75.48 \mathrm{MHz}, \mathrm{C}_{6} \mathrm{D}_{6}, 298 \mathrm{~K}\right)$ of $\mathrm{Rh}\left\{\eta^{3}-\mathrm{CH}_{2} \mathrm{CHCHCH}_{2} \mathrm{CH}(\mathrm{Bpin}) \operatorname{Pr}\right\}\left\{\kappa^{2}-\mathrm{P}, \mathrm{P}-\left[\operatorname{xant}\left(\mathrm{P}^{\mathrm{i}} \mathrm{Pr}_{2}\right)_{2}\right]\right\}(7)$. 

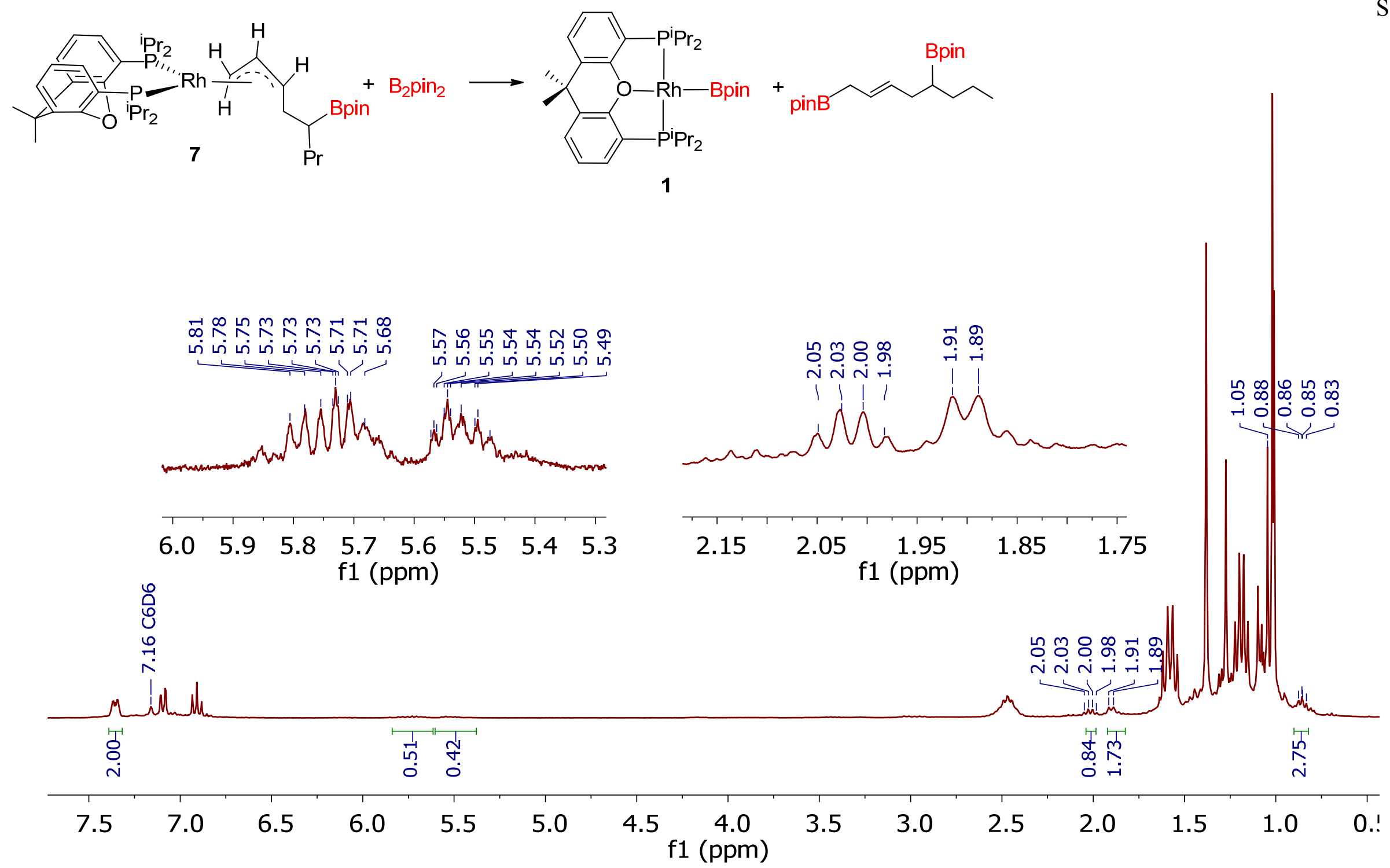

Figure S29. ${ }^{1} \mathrm{H}$ NMR spectrum $\left(300.13 \mathrm{MHz}, \mathrm{C}_{6} \mathrm{D}_{6}, 298 \mathrm{~K}\right)$ of the reaction of $\mathrm{Rh}\left\{\eta^{3}-\mathrm{CH}_{2} \mathrm{CHCHCH}(\mathrm{Bpin}) \operatorname{Pr}\right\}\left\{\kappa^{2}-\mathrm{P}, \mathrm{P}-\left[\mathrm{xant}\left(\mathrm{P}^{\mathrm{i}} \mathrm{Pr}_{2}\right)_{2}\right]\right\}(7)$ with $\mathrm{B}_{2} \mathrm{pin}_{2}$ to give complex 1 and 1,5-dipinacolboryl- $(E)$-2-octene. 


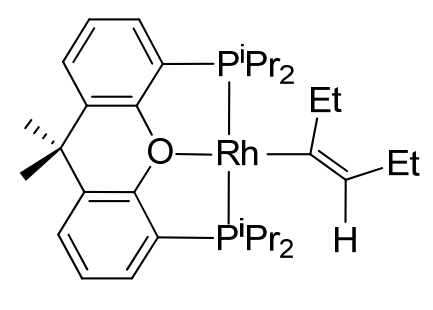

9

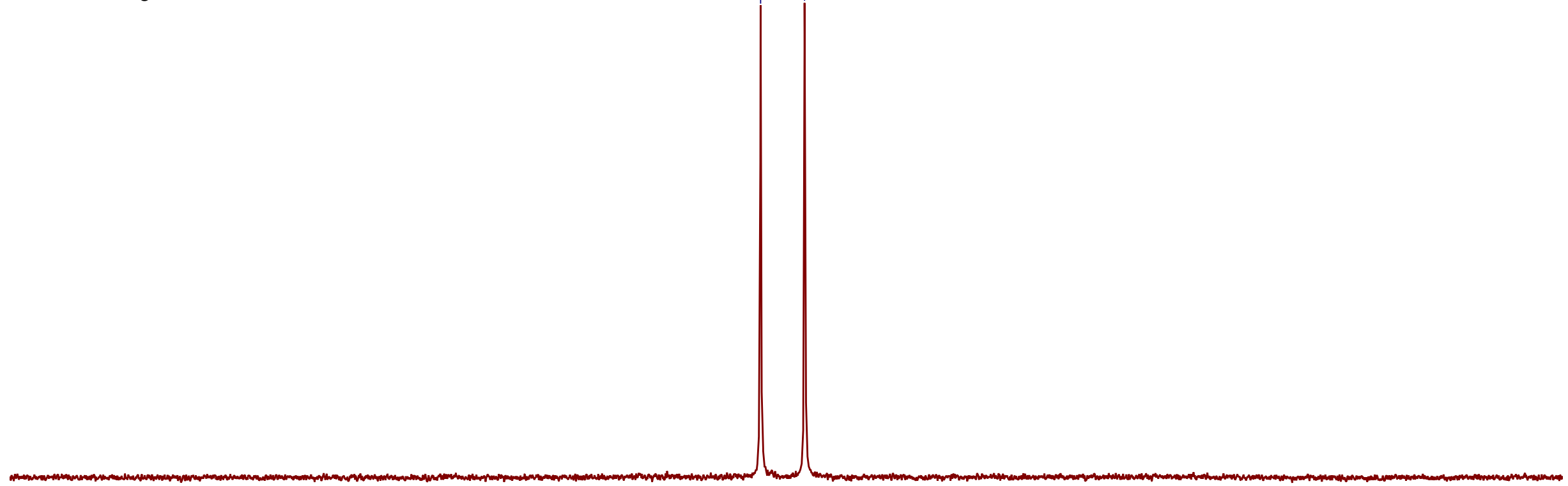

4847464544434241403938373635343332313029282726252423222120191817 f1 (ppm)

Figure S30. ${ }^{31} \mathrm{P}\left\{{ }^{1} \mathrm{H}\right\}$ NMR spectrum $\left(202.46 \mathrm{MHz}, \mathrm{C}_{6} \mathrm{D}_{6}, 298 \mathrm{~K}\right)$ of $\operatorname{Rh}\{(Z)-\mathrm{C}(\mathrm{Et})=\mathrm{C}(\mathrm{Et}) \mathrm{H}\}\left\{\kappa^{3}-\mathrm{P}, \mathrm{O}, \mathrm{P}-\left[\operatorname{xant}\left(\mathrm{P}^{\mathrm{i}} \mathrm{Pr}_{2}\right)_{2}\right]\right\}(9)$. 


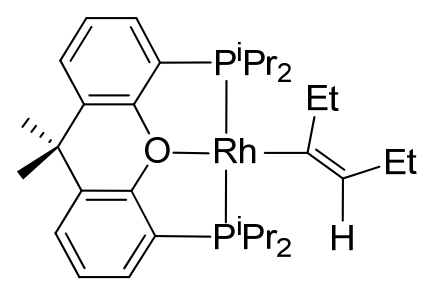

9

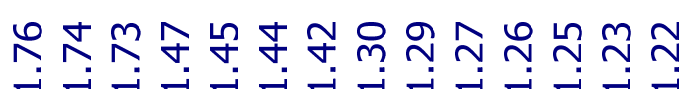

ri-i -

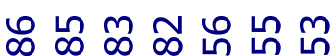
กั่ง

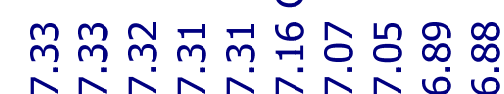

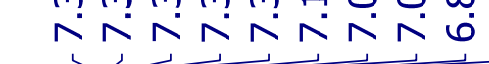

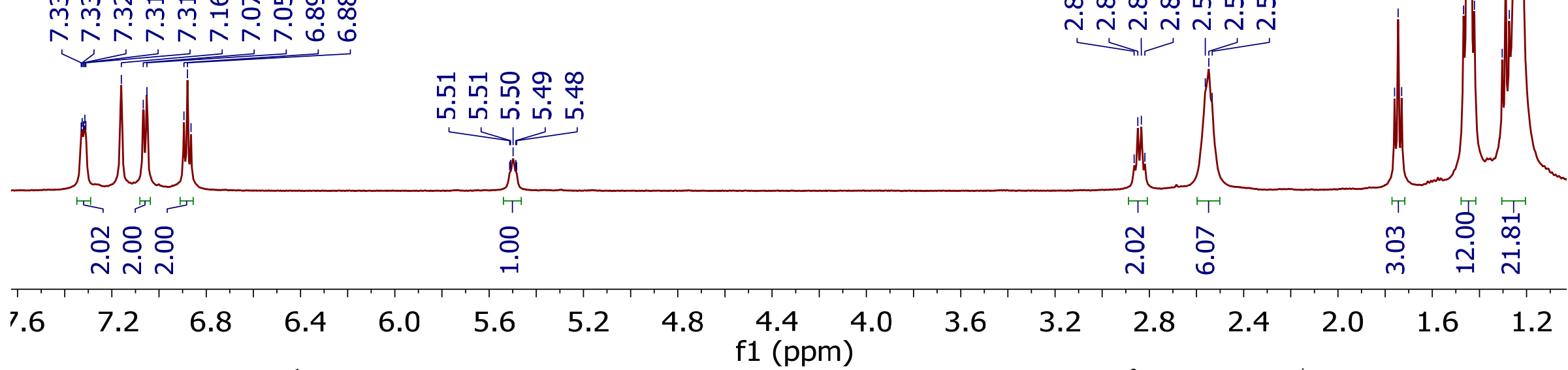

Figure S31. ${ }^{1} \mathrm{H}$ NMR spectrum $\left(500.12 \mathrm{MHz}, \mathrm{C}_{6} \mathrm{D}_{6}, 298 \mathrm{~K}\right)$ of $\mathrm{Rh}\{(Z)-\mathrm{C}(\mathrm{Et})=\mathrm{C}(\mathrm{Et}) \mathrm{H}\}\left\{\kappa^{3}-\mathrm{P}, \mathrm{O}, \mathrm{P}-\left[\operatorname{xant}\left(\mathrm{P}^{\mathrm{i}} \operatorname{Pr}_{2}\right)_{2}\right]\right\}(\mathbf{9})$. 


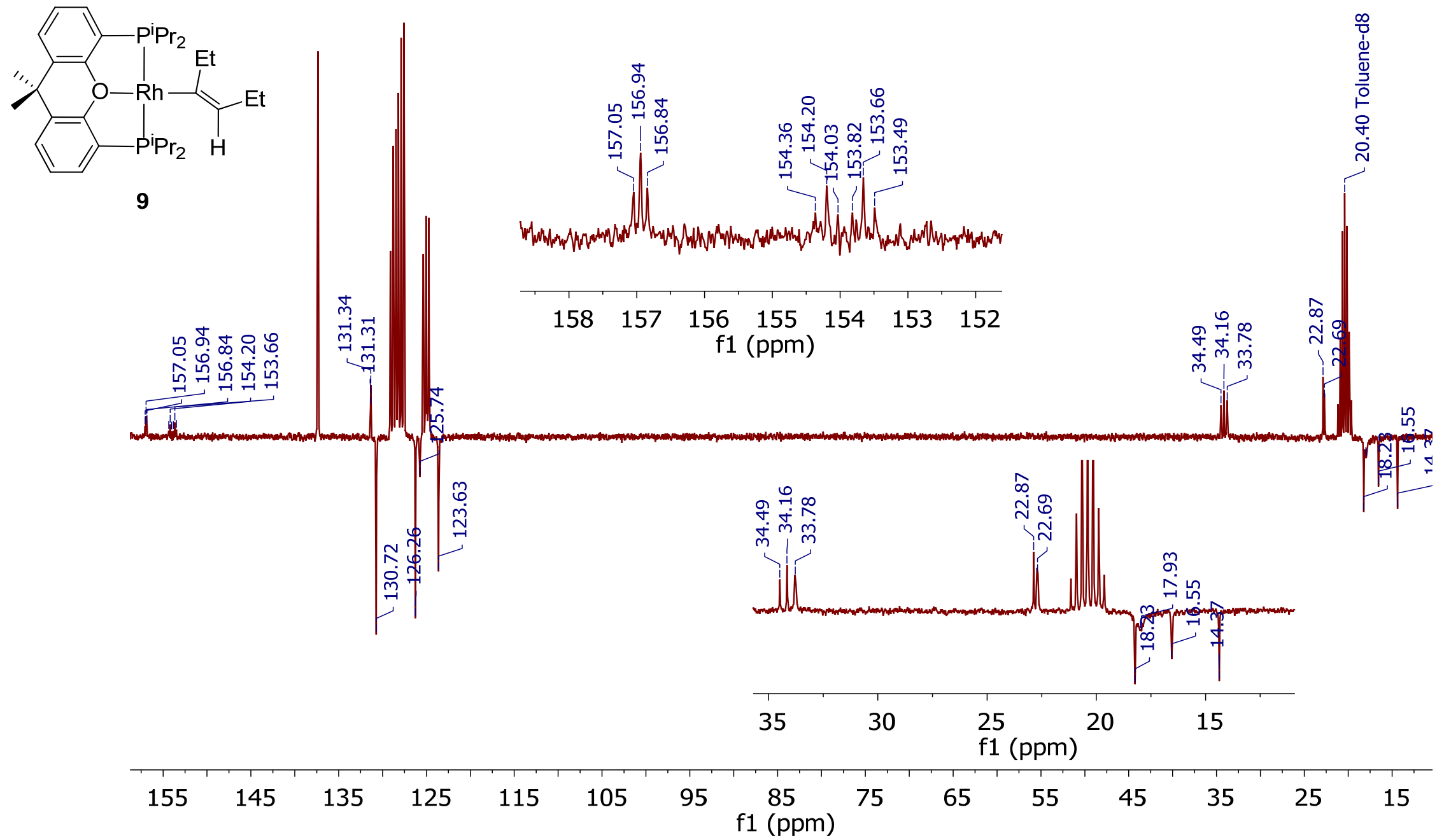

Figure S32. ${ }^{13} \mathrm{C}\left\{{ }^{1} \mathrm{H}\right\}$-apt NMR spectrum $\left(75.48 \mathrm{MHz}, \mathrm{C}_{7} \mathrm{D}_{8}, 253 \mathrm{~K}\right)$ of $\mathrm{Rh}\{(\mathrm{Z})-\mathrm{C}(\mathrm{Et})=\mathrm{C}(\mathrm{Et}) \mathrm{H}\}\left\{\kappa^{3}-\mathrm{P}, \mathrm{O}, \mathrm{P}-\left[\operatorname{xant}\left(\mathrm{P}^{\mathrm{i}} \mathrm{Pr}_{2}\right)_{2}\right]\right\}(9)$. 


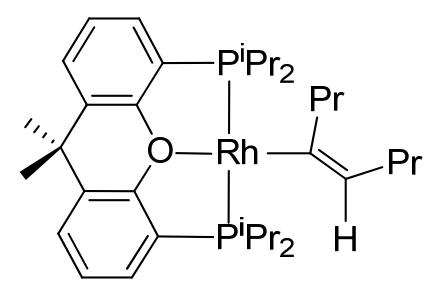

10

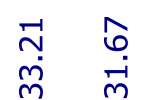

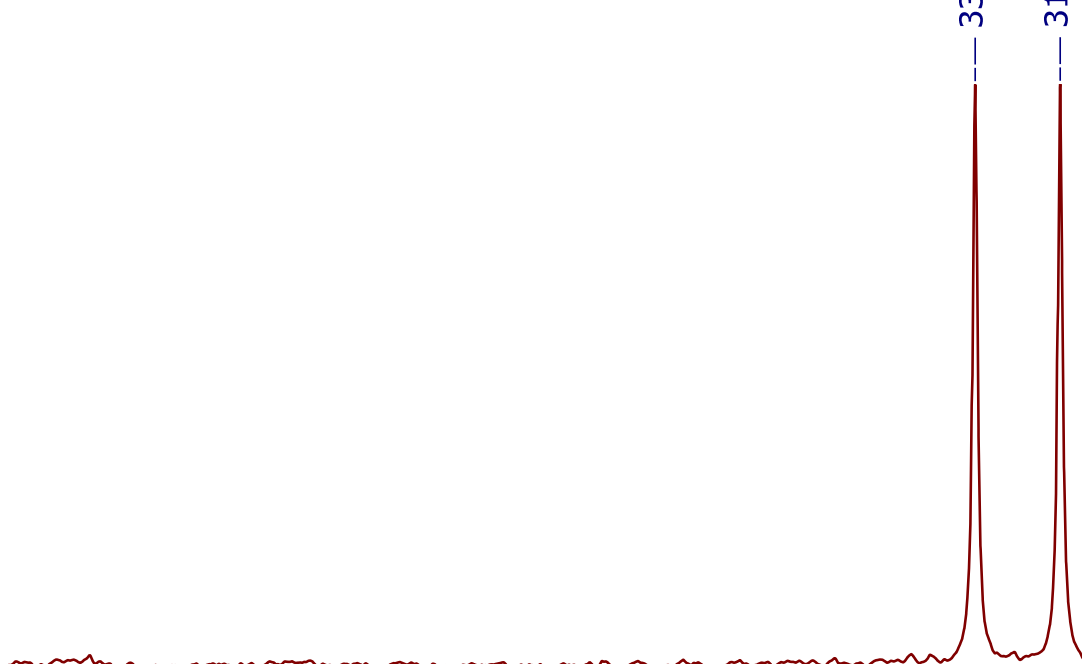

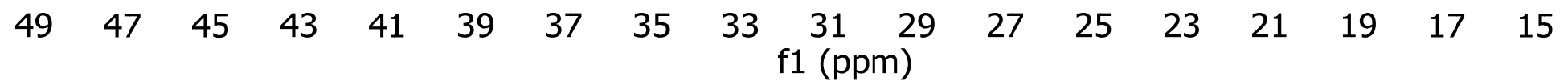

Figure S33. ${ }^{31} \mathrm{P}\left\{{ }^{1} \mathrm{H}\right\}$ NMR spectrum $\left(121.49 \mathrm{MHz}, \mathrm{C}_{6} \mathrm{D}_{6}, 298 \mathrm{~K}\right)$ of $\mathrm{Rh}\{(Z)-\mathrm{C}(\mathrm{Pr})=\mathrm{C}(\mathrm{Pr}) \mathrm{H}\}\left\{\kappa^{3}-\mathrm{P}, \mathrm{O}, \mathrm{P}-\left[\mathrm{xant}\left(\mathrm{P}^{\mathrm{i}} \mathrm{Pr}_{2}\right)_{2}\right]\right\}(\mathbf{1 0})$. 


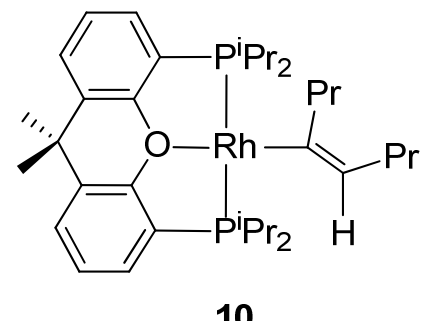

10

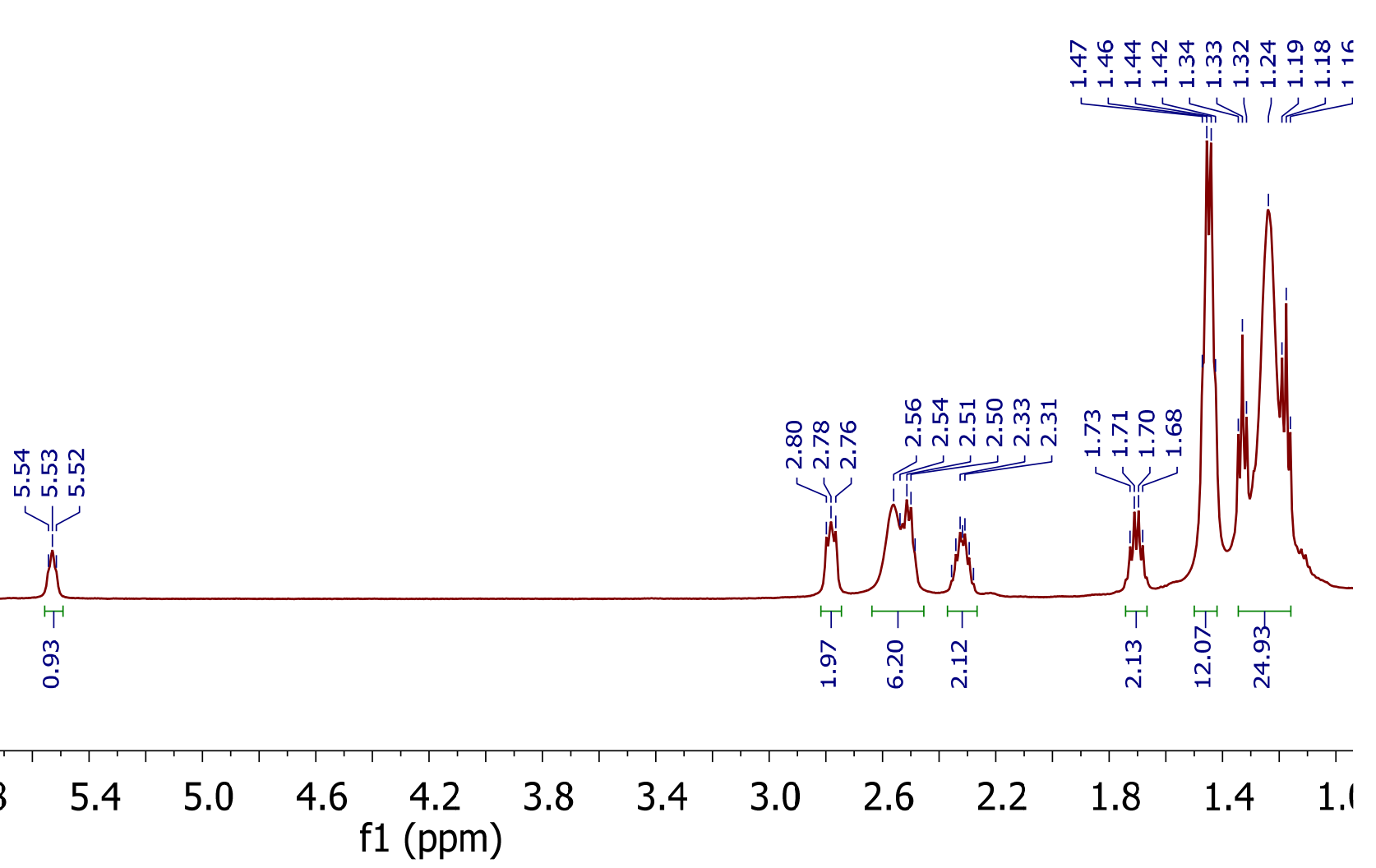

Figure S34. ${ }^{1} \mathrm{H}$ NMR spectrum $\left(500.13 \mathrm{MHz}, \mathrm{C}_{6} \mathrm{D}_{6}, 298 \mathrm{~K}\right)$ of $\mathrm{Rh}\{(Z)-\mathrm{C}(\mathrm{Pr})=\mathrm{C}(\mathrm{Pr}) \mathrm{H}\}\left\{\kappa^{3}-\mathrm{P}, \mathrm{O}, \mathrm{P}-\left[\operatorname{xant}\left(\mathrm{P}^{\mathrm{i}} \operatorname{Pr}_{2}\right)_{2}\right]\right\}(\mathbf{1 0})$. 


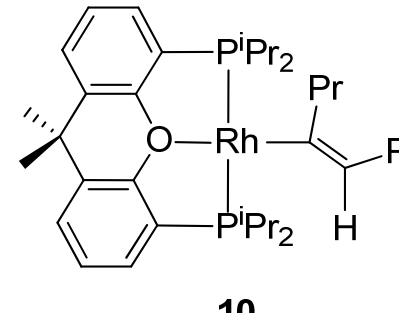

10
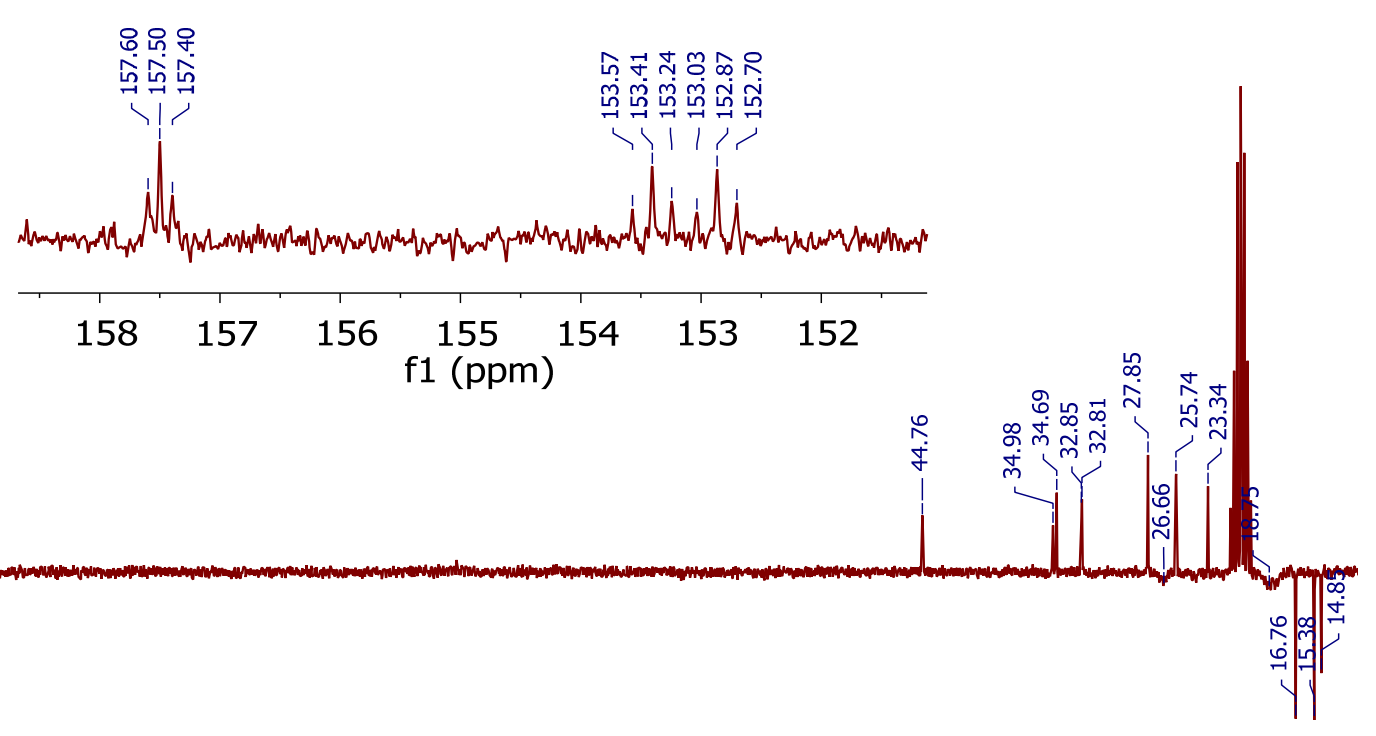

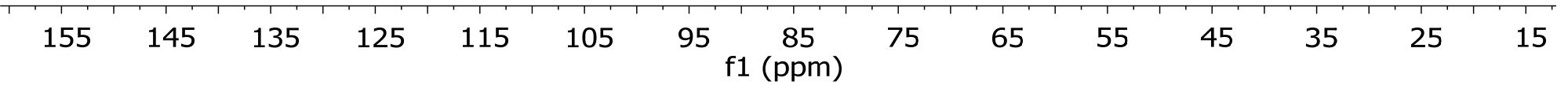

Figure S35. ${ }^{13} \mathrm{C}\left\{{ }^{1} \mathrm{H}\right\}$ NMR spectrum $\left(75.48 \mathrm{MHz}\right.$, tol- $\left.\mathrm{d}_{8}, 253 \mathrm{~K}\right)$ of $\operatorname{Rh}\{(Z)-\mathrm{C}(\mathrm{Pr})=\mathrm{C}(\operatorname{Pr}) \mathrm{H}\}\left\{\kappa^{3}-\mathrm{P}, \mathrm{O}, \mathrm{P}-\left[\operatorname{xant}\left(\mathrm{P}^{\mathrm{i}} \operatorname{Pr}_{2}\right)_{2}\right]\right\}(\mathbf{1 0})$. 

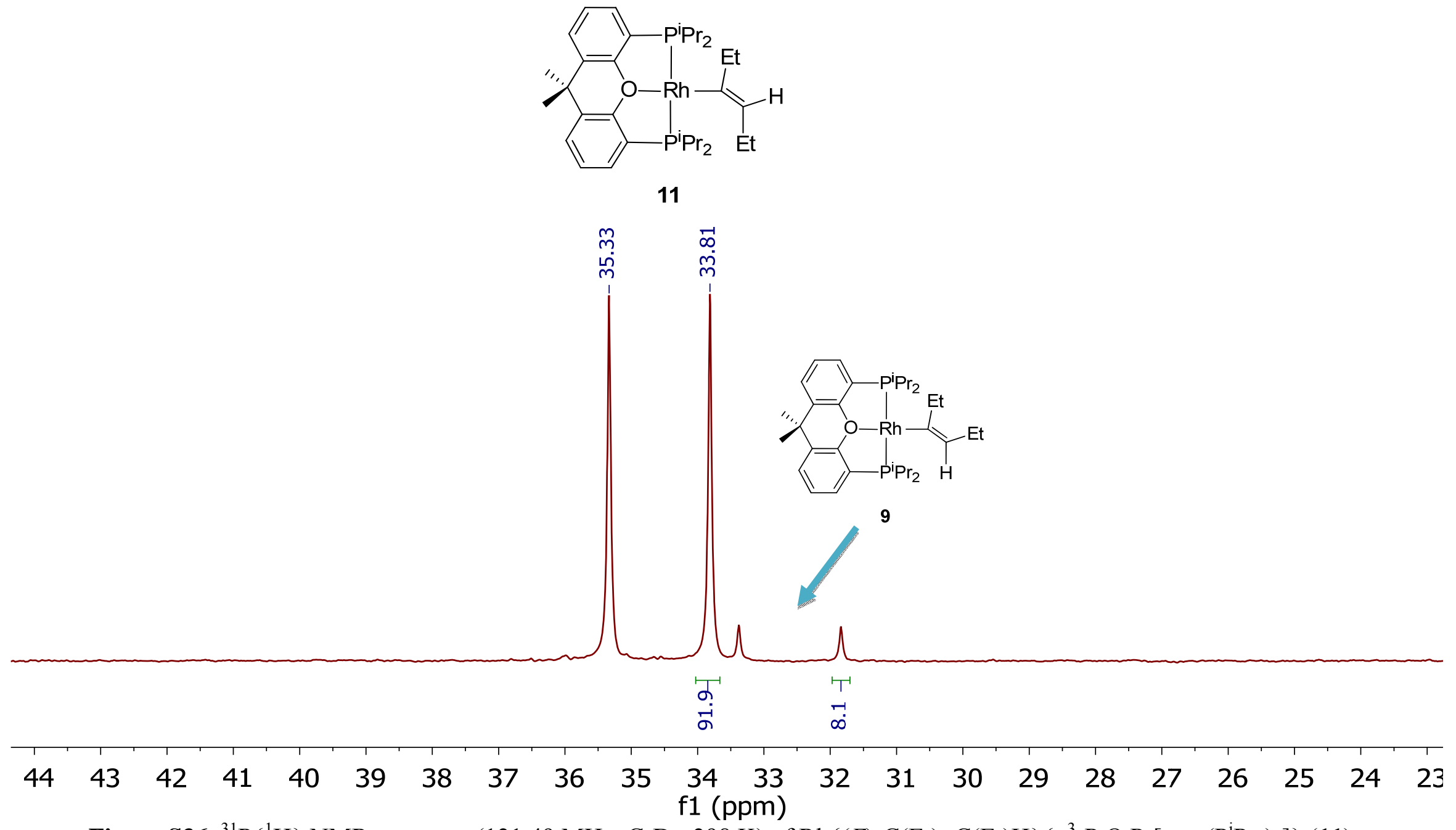

Figure S36. ${ }^{31} \mathrm{P}\left\{{ }^{1} \mathrm{H}\right\}$ NMR spectrum $\left(121.49 \mathrm{MHz}, \mathrm{C}_{6} \mathrm{D}_{6}, 298 \mathrm{~K}\right)$ of $\mathrm{Rh}\{(E)-\mathrm{C}(\mathrm{Et})=\mathrm{C}(\mathrm{Et}) \mathrm{H}\}\left\{\kappa^{3}-\mathrm{P}, \mathrm{O}, \mathrm{P}-\left[\operatorname{xant}\left(\mathrm{P}^{\mathrm{i}} \mathrm{Pr}_{2}\right)_{2}\right]\right\}(\mathbf{1 1})$. 


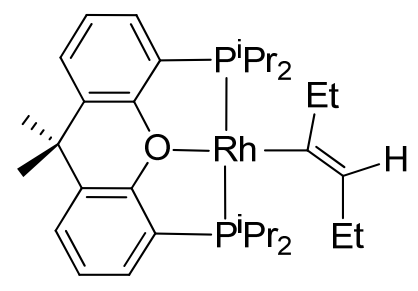

11

రి

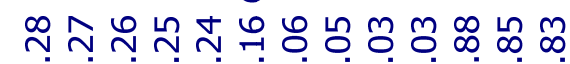

กักง

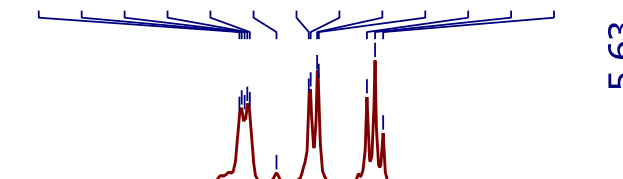

计蛙

๕ัฒ

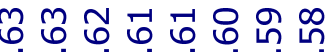

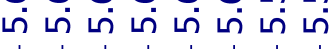
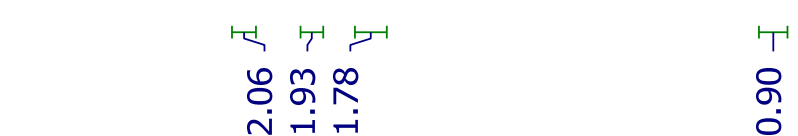

'T

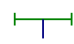

$T$

$\stackrel{+}{\sim}$

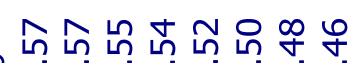

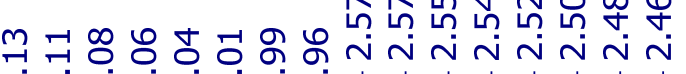
mं mं mं mं
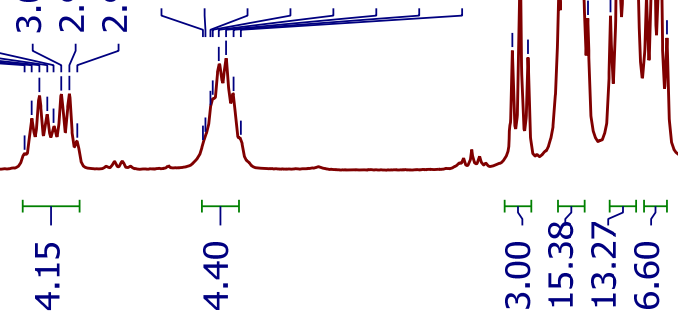

\begin{tabular}{|c|c|c|c|c|c|c|c|c|c|c|c|c|c|c|}
\hline 0 & 7.5 & 7.0 & 6.5 & 6.0 & 5.5 & 5.0 & $\begin{array}{c}4.5 \\
f 1(p p m\end{array}$ & 4.0 & 3.5 & 3.0 & 2.5 & 2.0 & 1.5 & 1.0 \\
\hline
\end{tabular}

Figure S37. ${ }^{1} \mathrm{H}$ NMR spectrum $\left(300.13 \mathrm{MHz}, \mathrm{C}_{6} \mathrm{D}_{6}, 298 \mathrm{~K}\right)$ of $\mathrm{Rh}\{(E)-\mathrm{C}(\mathrm{Et})=\mathrm{C}(\mathrm{Et}) \mathrm{H}\}\left\{\kappa^{3}-\mathrm{P}, \mathrm{O}, \mathrm{P}-\left[\mathrm{xant}\left(\mathrm{P}^{\mathrm{i}} \mathrm{Pr}_{2}\right)_{2}\right]\right\}(\mathbf{1 1})$. 


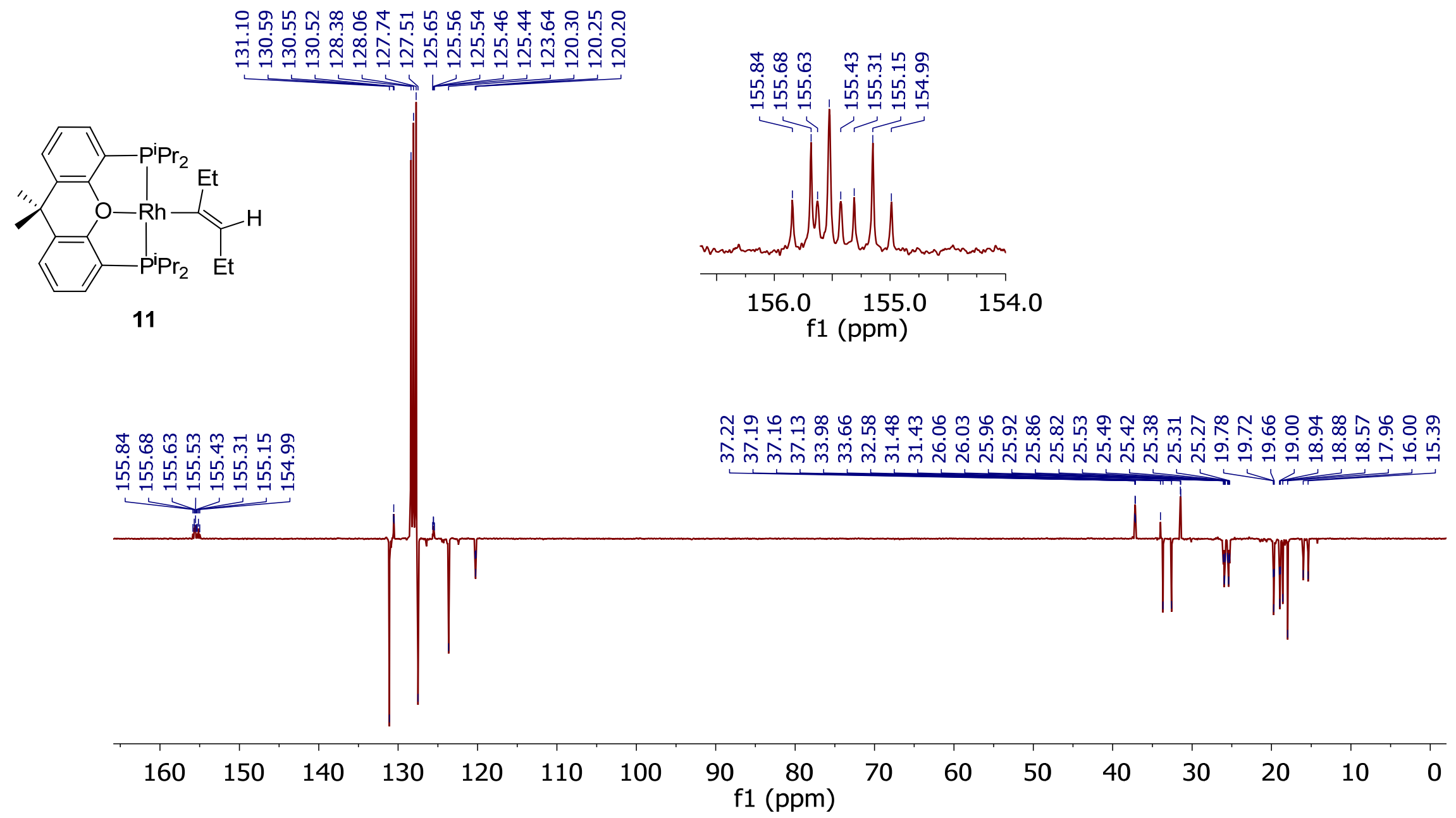

Figure S38. ${ }^{13} \mathrm{C}\left\{{ }^{1} \mathrm{H}\right\}$ NMR-apt NMR spectrum $\left(75.48 \mathrm{MHz}, \mathrm{C}_{6} \mathrm{D}_{6}, 298 \mathrm{~K}\right)$ of $\mathrm{Rh}\{(E)-\mathrm{C}(\mathrm{Et})=\mathrm{C}(\mathrm{Et}) \mathrm{H}\}\left\{\kappa^{3}-\mathrm{P}, \mathrm{O}, \mathrm{P}-\left[\operatorname{xant}\left(\mathrm{P}^{\mathrm{i}} \mathrm{Pr}_{2}\right)_{2}\right]\right\}(\mathbf{1 1})$. 


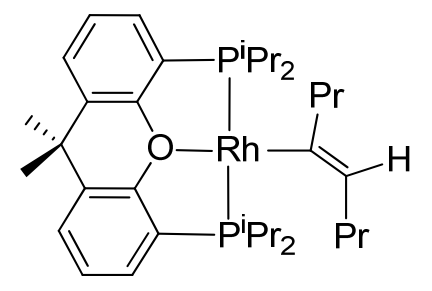

12

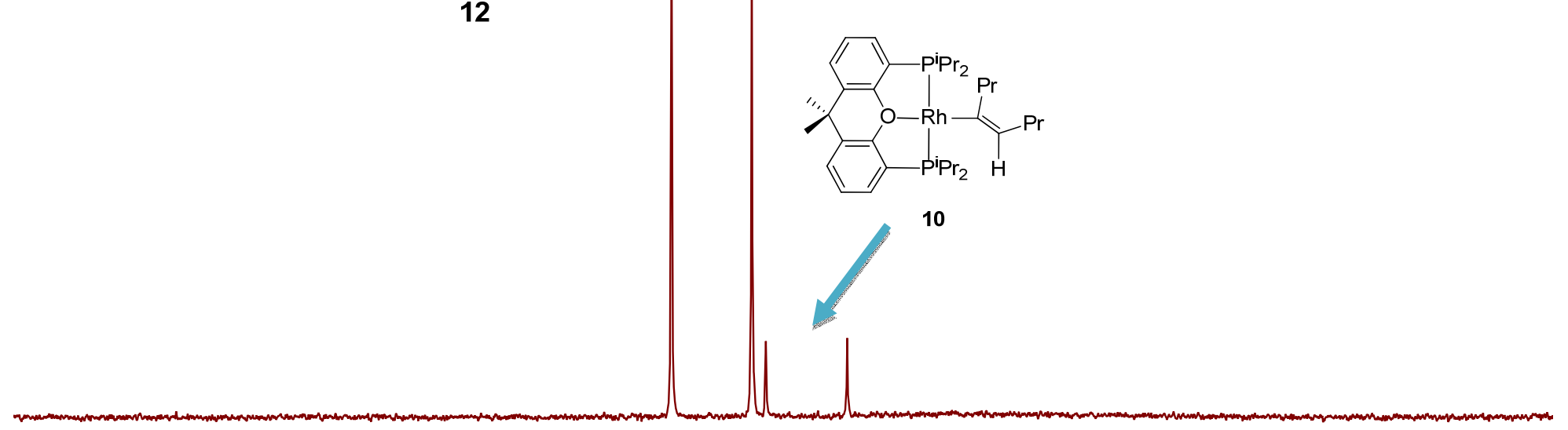

$\begin{array}{lllllllllllllllllllllllllllll}47 & 46 & 45 & 44 & 43 & 42 & 41 & 40 & 39 & 38 & 37 & 36 & 35 & 34 & 33 & 32 & 31 & 30 & 29 & 28 & 27 & 26 & 25 & 24 & 23 & 22 & 21 & 20 & 19\end{array}$ f1 (ppm)

Figure S39. ${ }^{31} \mathrm{P}\left\{{ }^{1} \mathrm{H}\right\}$ NMR spectrum $\left(121.49 \mathrm{MHz}, \mathrm{C}_{6} \mathrm{D}_{6}, 298 \mathrm{~K}\right)$ of the reaction of $\mathrm{RhH}\left\{\kappa^{3}-\mathrm{P}, \mathrm{O}, \mathrm{P}-\left[\operatorname{xant}\left(\mathrm{P}^{\mathrm{i}} \mathrm{Pr}_{2}\right)_{2}\right]\right\}(\mathbf{8})$ with 4-octyne: Spectroscopic detection of of $\mathrm{Rh}\{(E)-\mathrm{C}(\mathrm{Pr})=\mathrm{C}(\mathrm{Pr}) \mathrm{H}\}\left\{\kappa^{3}-\mathrm{P}, \mathrm{O}, \mathrm{P}-\left[\operatorname{xant}\left(\mathrm{P}^{\mathrm{i}} \operatorname{Pr}_{2}\right)_{2}\right]\right\}(\mathbf{1 2})$. 

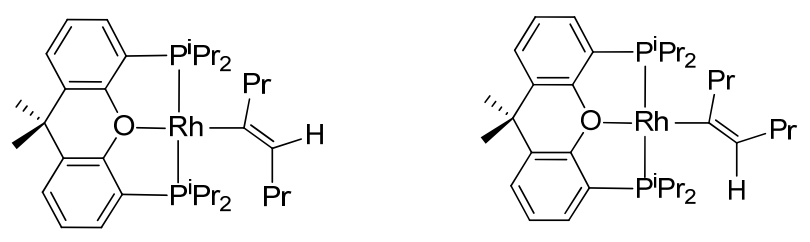

12

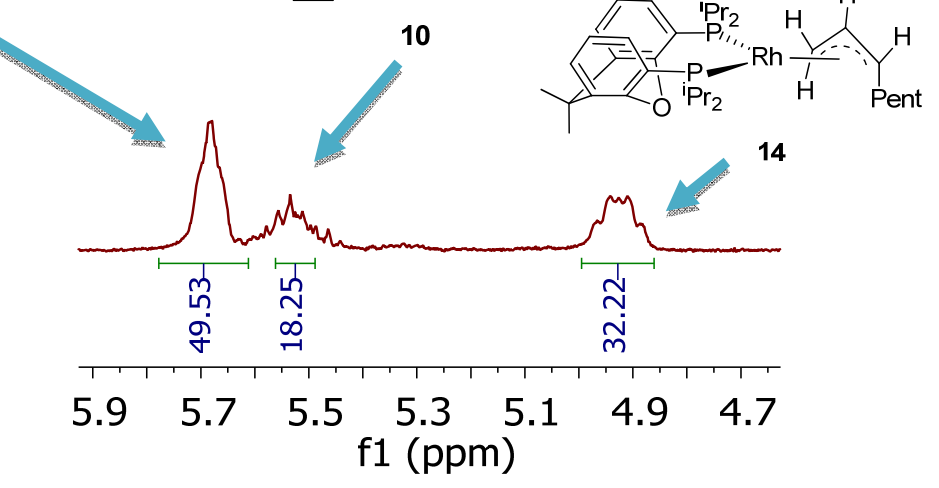

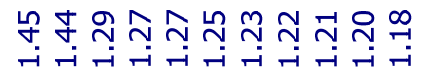

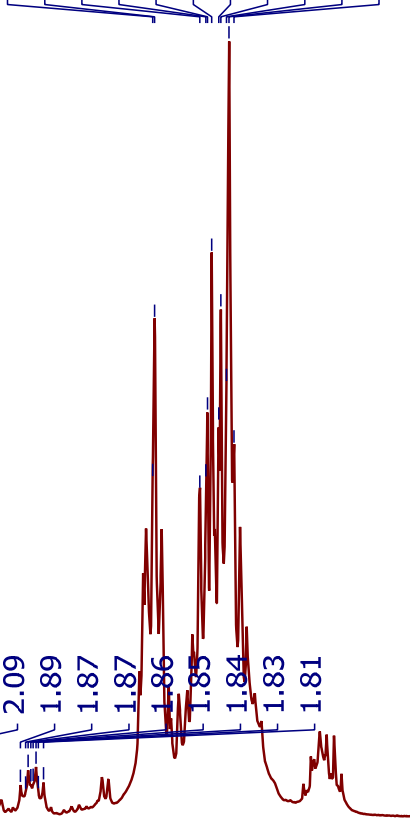

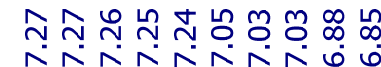

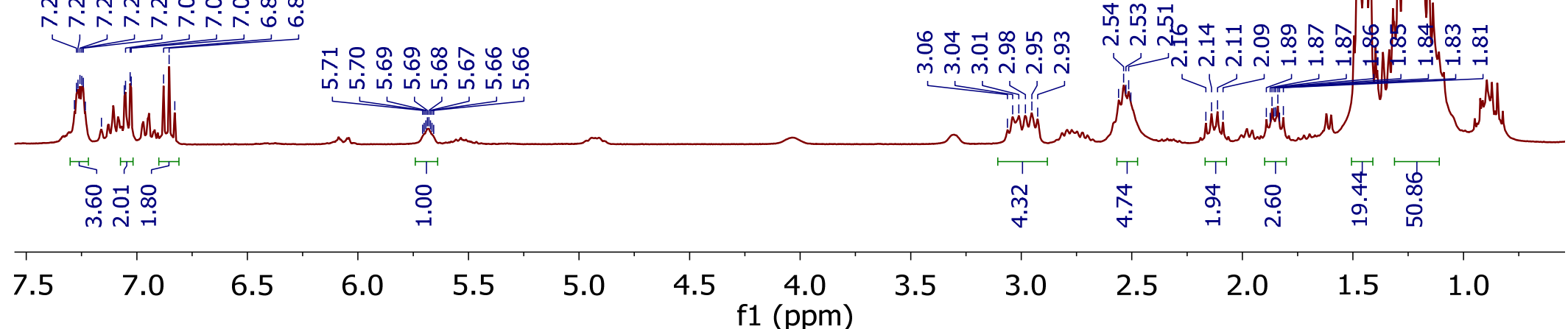

Figure S40. ${ }^{1} \mathrm{H}$ NMR spectrum $\left(300.13 \mathrm{MHz}, \mathrm{C}_{6} \mathrm{D}_{6}, 298 \mathrm{~K}\right)$ of the reaction of $\mathrm{RhH}\left\{\kappa^{3}-\mathrm{P}, \mathrm{O}, \mathrm{P}-\left[\mathrm{xant}\left(\mathrm{P}^{\mathrm{i}} \mathrm{Pr}_{2}\right)_{2}\right]\right\}$ (8) with 4-octyne: Spectroscopic detection of of $\mathrm{Rh}\{(E)-\mathrm{C}(\mathrm{Pr})=\mathrm{C}(\mathrm{Pr}) \mathrm{H}\}\left\{\kappa^{3}-\mathrm{P}, \mathrm{O}, \mathrm{P}-\left[\operatorname{xant}\left(\mathrm{P}^{\mathrm{i}} \mathrm{Pr}_{2}\right)_{2}\right]\right\}$ (12). 


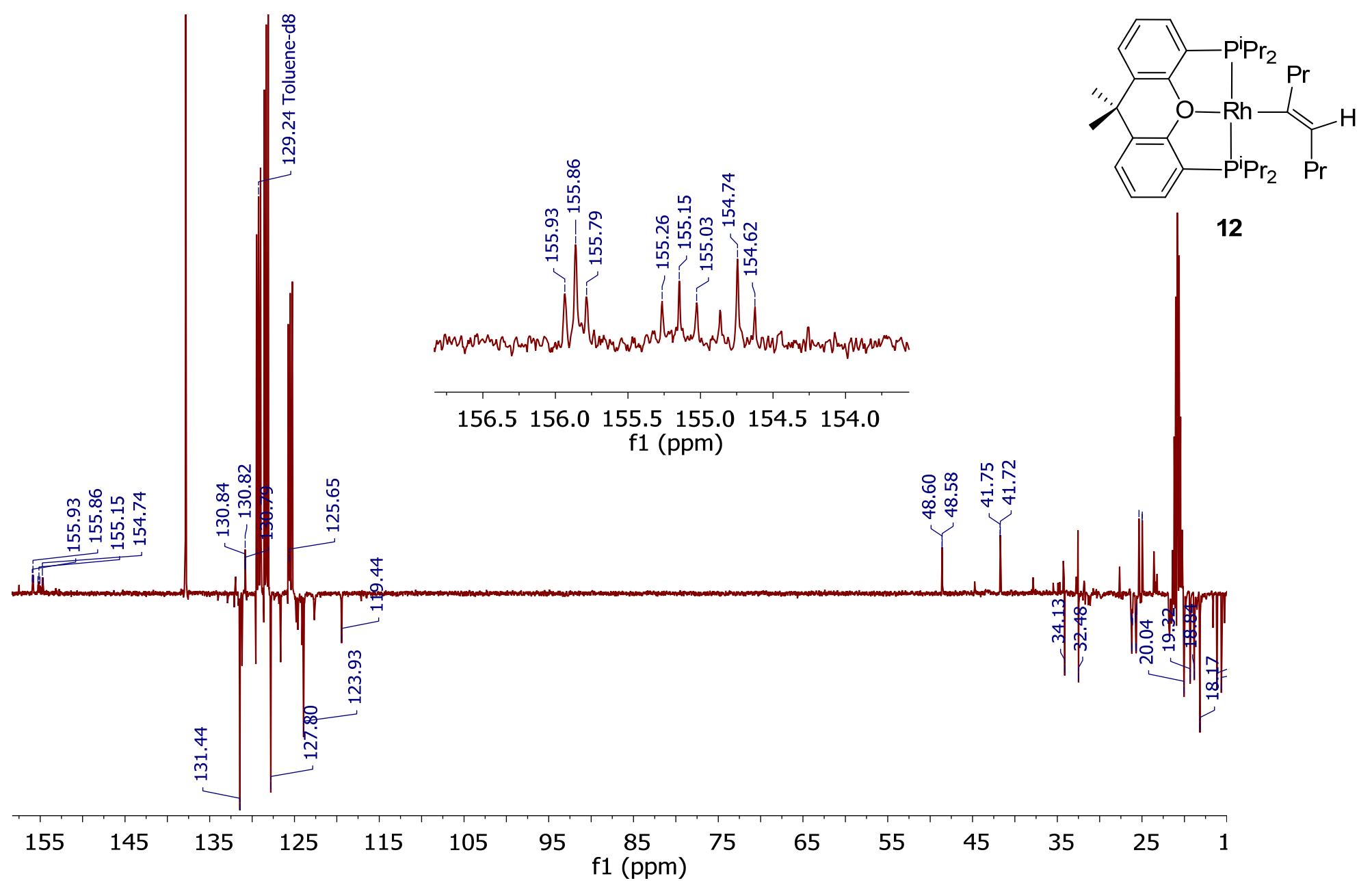

Figure S41. ${ }^{13} \mathrm{C}\left\{{ }^{1} \mathrm{H}\right\}$ NMR-apt NMR spectrum $\left(100.62 \mathrm{MHz}\right.$, toluene- $\left.\mathrm{d}_{8}, 273 \mathrm{~K}\right)$ of the reaction of $\mathrm{RhH}\left\{\kappa^{3}-\mathrm{P}, \mathrm{O}, \mathrm{P}-\left[\mathrm{xant}\left(\mathrm{P}^{\mathrm{i}} \mathrm{Pr}_{2}\right)_{2}\right]\right\}(8)$ with 4octyne: Spectroscopic detection of of $\mathrm{Rh}\{(E)-\mathrm{C}(\mathrm{Pr})=\mathrm{C}(\mathrm{Pr}) \mathrm{H}\}\left\{\kappa^{3}-\mathrm{P}, \mathrm{O}, \mathrm{P}-\left[\operatorname{xant}\left(\mathrm{P}^{\mathrm{i}} \mathrm{Pr}_{2}\right)_{2}\right]\right\}$ (12). 
${ }^{31} \mathrm{P}\left\{{ }^{1} \mathrm{H}\right\} \mathrm{NMR}$ at $298 \mathrm{~K}$

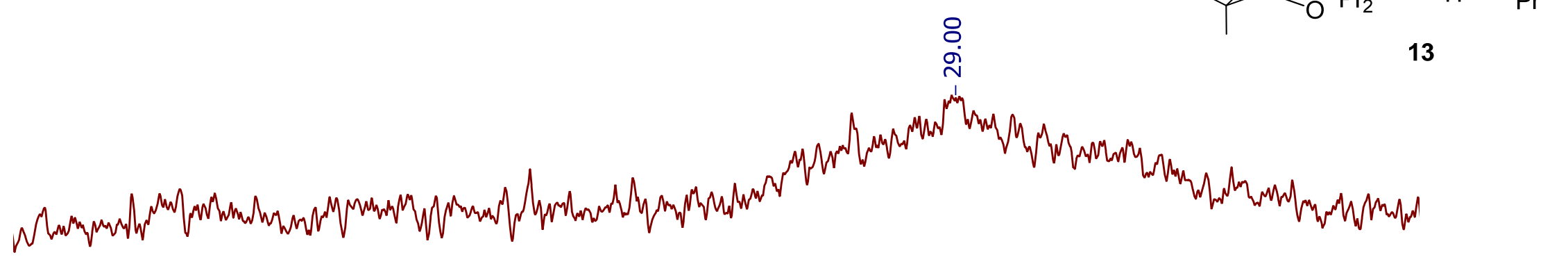

${ }^{31} \mathrm{P}\left\{{ }^{1} \mathrm{H}\right\} \mathrm{NMR}$ at $193 \mathrm{~K}$
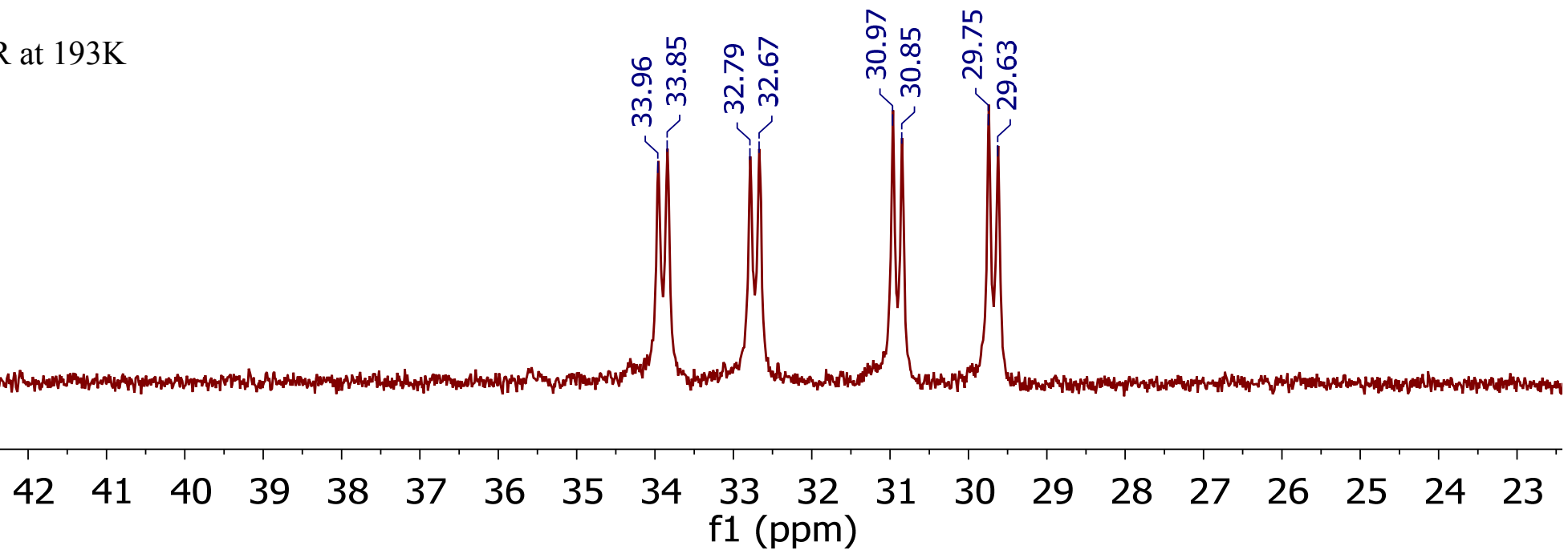

Figure S42. ${ }^{31} \mathrm{P}\left\{{ }^{1} \mathrm{H}\right\}$ NMR spectra $\left(161.98 \mathrm{MHz}\right.$, toluene- $\left.d_{8}\right)$ of $\mathrm{Rh}\left(\eta^{3}-\mathrm{CH}_{2} \mathrm{CHCHPr}\right)\left\{\kappa^{2}-\mathrm{P}, \mathrm{P}-\left[\mathrm{xant}\left(\mathrm{P}^{\mathrm{i}} \mathrm{Pr}_{2}\right)_{2}\right]\right\}(\mathbf{1 3})$ at 298 and $193 \mathrm{~K}$. 

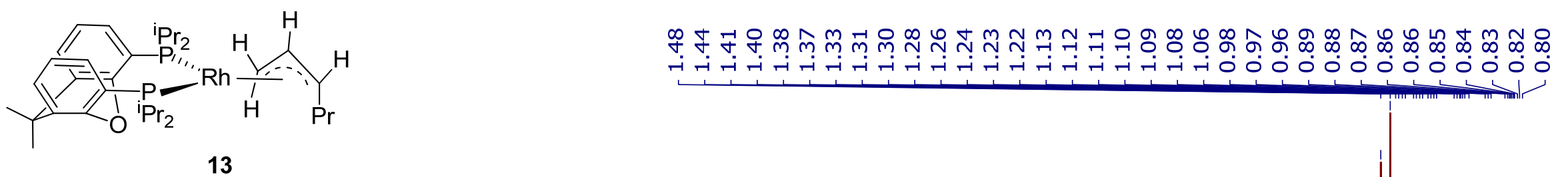

13

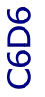

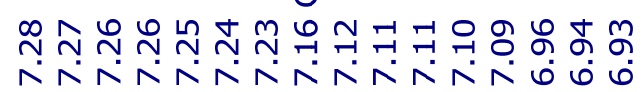

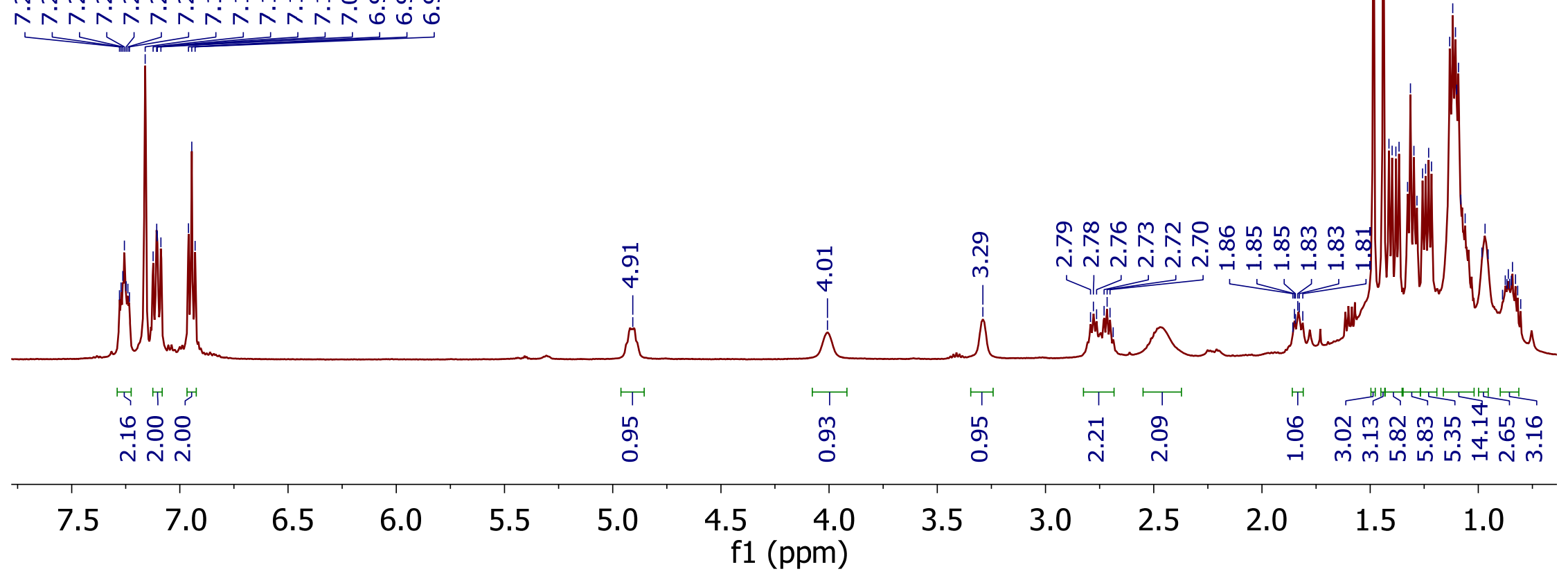

Figure S43. ${ }^{1} \mathrm{H}$ NMR spectrum $\left(500.12 \mathrm{MHz}, \mathrm{C}_{6} \mathrm{D}_{6}, 298 \mathrm{~K}\right)$ of $\mathrm{Rh}\left(\eta^{3}-\mathrm{CH}_{2} \mathrm{CHCHPr}\right)\left\{\kappa^{2}-\mathrm{P}, \mathrm{P}-\left[\operatorname{xant}\left(\mathrm{P}^{\mathrm{i}} \mathrm{Pr}_{2}\right)_{2}\right]\right\}(\mathbf{1 3})$. 


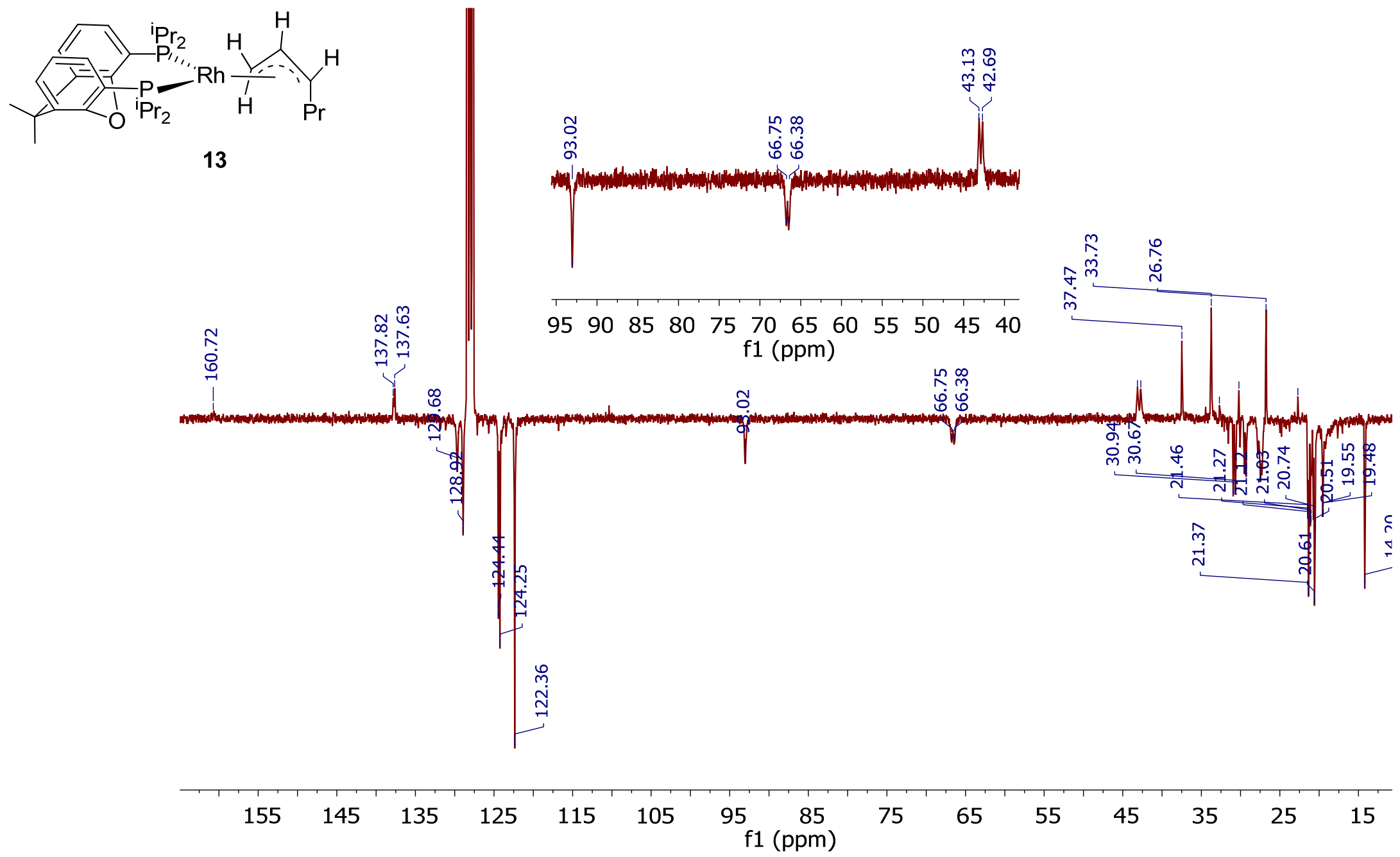

Figure S44. ${ }^{13} \mathrm{C}\left\{{ }^{1} \mathrm{H}\right\}$-apt NMR spectrum $\left(75.48 \mathrm{MHz}, \mathrm{C}_{6} \mathrm{D}_{6}, 298 \mathrm{~K}\right)$ of $\mathrm{Rh}\left(\eta^{3}-\mathrm{CH}_{2} \mathrm{CHCHPr}\right)\left\{\kappa^{2}-\mathrm{P}, \mathrm{P}-\left[\operatorname{xant}\left(\mathrm{P}^{\mathrm{i}} \mathrm{Pr}_{2}\right)_{2}\right]\right\}(\mathbf{1 3})$. 
${ }^{31} \mathrm{P}\left\{{ }^{1} \mathrm{H}\right\} \mathrm{NMR}$ at $298 \mathrm{~K}$

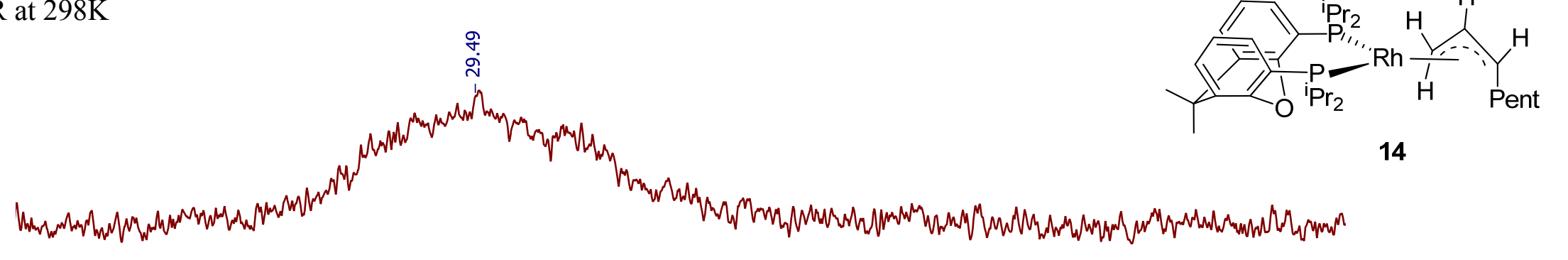

${ }^{31} \mathrm{P}\left\{{ }^{1} \mathrm{H}\right\} \mathrm{NMR}$ at $193 \mathrm{~K}$

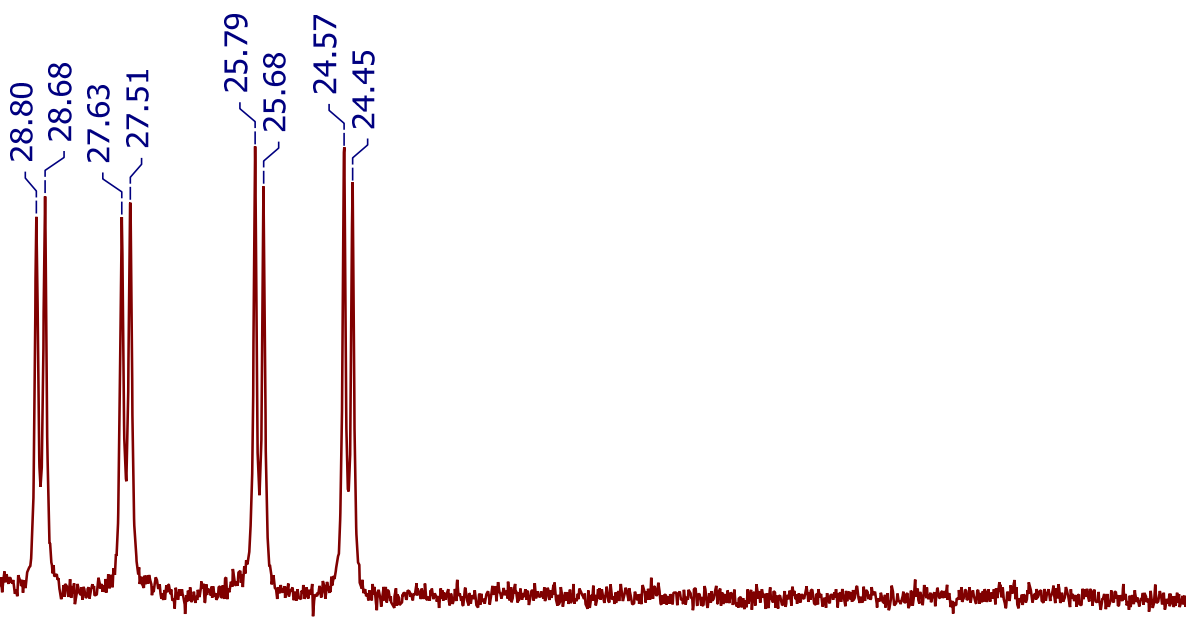

$\begin{array}{llllllllllllllllllllllllllll}40 & 39 & 38 & 37 & 36 & 35 & 34 & 33 & 32 & 31 & 30 & 29 & 28 & 27 & 26 & 25 & 24 & 23 & 22 & 21 & 20 & 19 & 18 & 17 & 16 & 15 & 14 & 1\end{array}$

Figure S45. ${ }^{31} \mathrm{P}\left\{{ }^{1} \mathrm{H}\right\}$ NMR spectra $\left(161.98 \mathrm{MHz}\right.$, toluene- $\left.d_{8}\right)$ of $\mathrm{Rh}\left(\eta^{3}-\mathrm{CH}_{2} \mathrm{CHCHPent}\right)\left\{\kappa^{2}-\mathrm{P}, \mathrm{P}-\left[\mathrm{xant}\left(\mathrm{P}^{\mathrm{i}} \mathrm{Pr}_{2}\right)_{2}\right]\right\}(\mathbf{1 4})$ at 298 and $193 \mathrm{~K}$. 


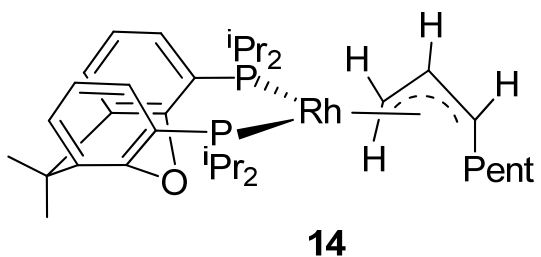

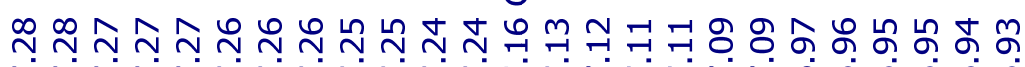

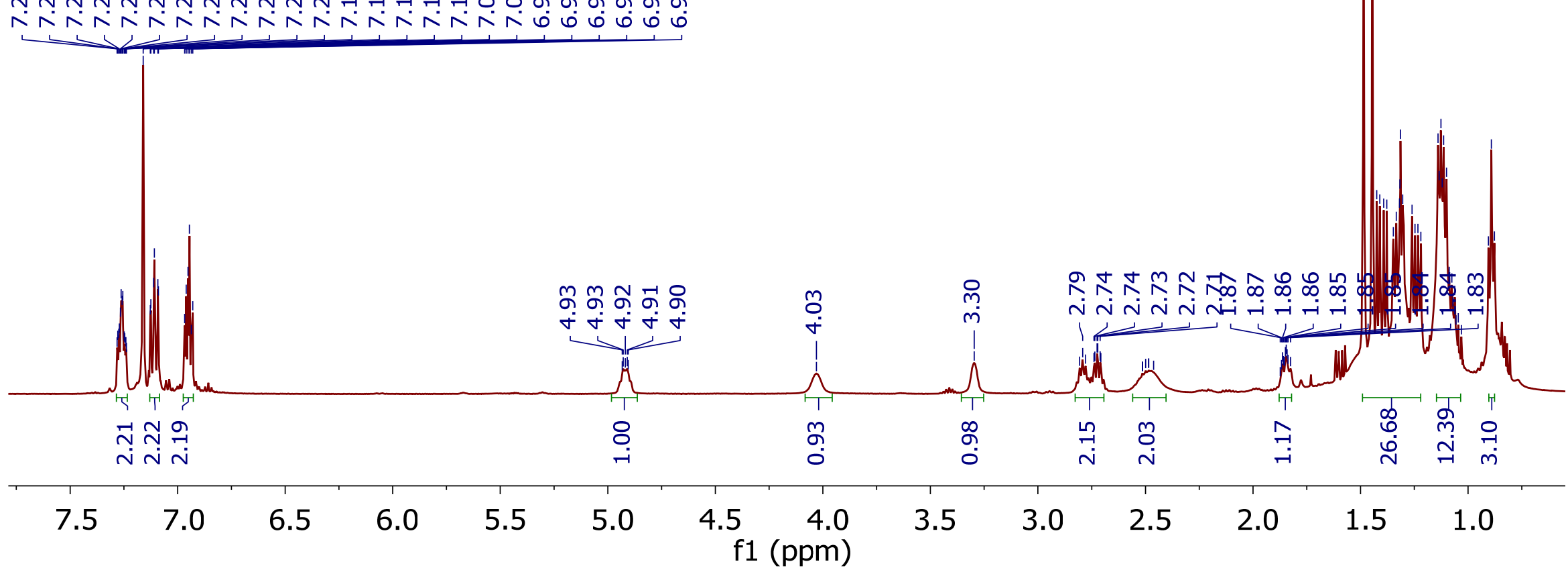

Figure S46. ${ }^{1} \mathrm{H}$ NMR spectrum $\left(500.12 \mathrm{MHz}, \mathrm{C}_{6} \mathrm{D}_{6}, 298 \mathrm{~K}\right)$ of $\mathrm{Rh}\left(\eta^{3}-\mathrm{CH}_{2} \mathrm{CHCHPent}\right)\left\{\kappa^{2}-\mathrm{P}, \mathrm{P}-\left[\mathrm{xant}\left(\mathrm{P}^{\mathrm{i}} \mathrm{Pr}_{2}\right)_{2}\right]\right\}(\mathbf{1 4})$. 


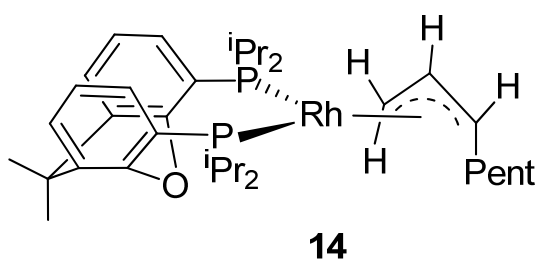

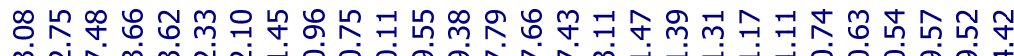

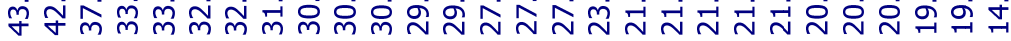

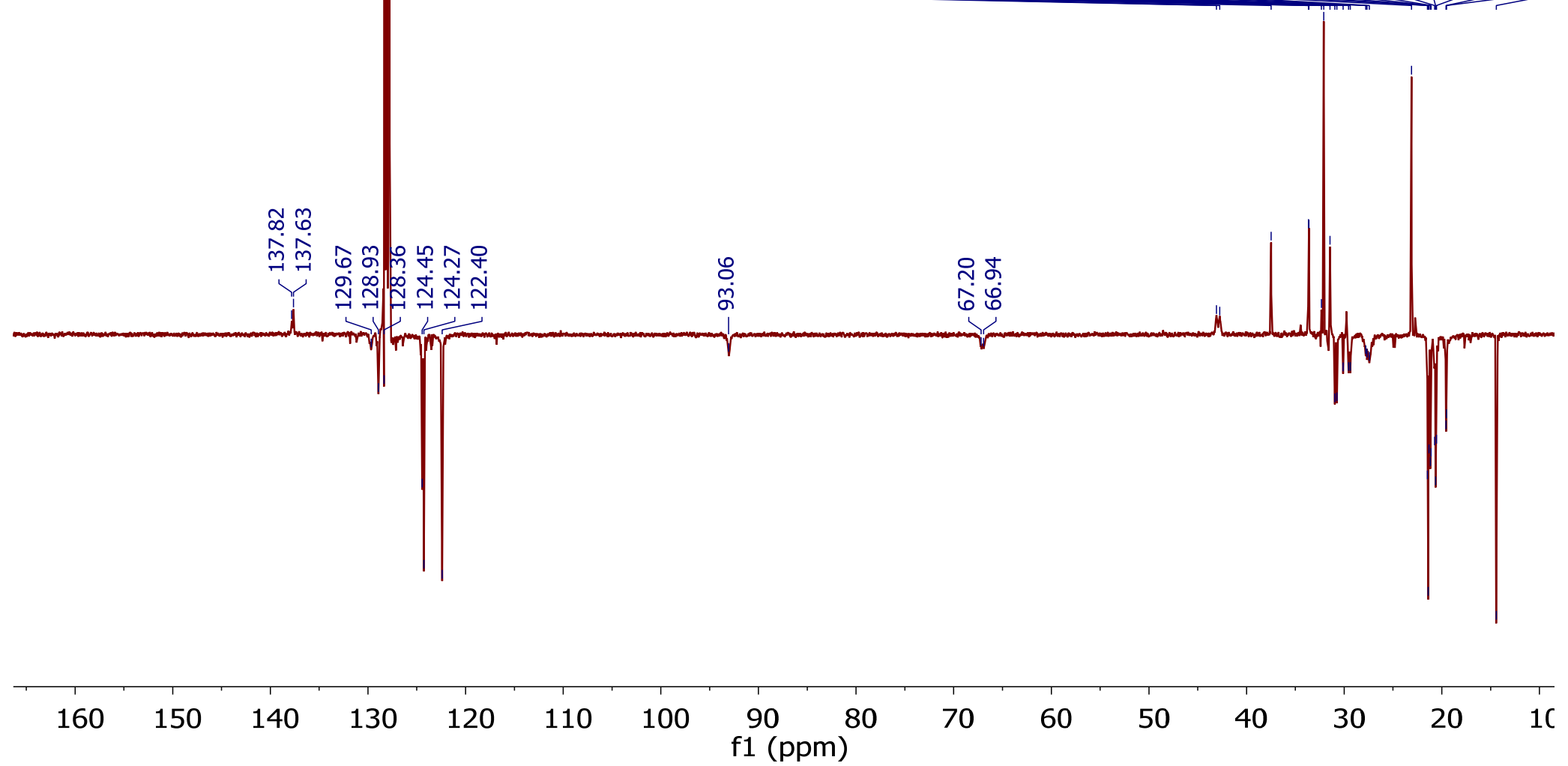

Figure S47. ${ }^{13} \mathrm{C}\left\{{ }^{1} \mathrm{H}\right\}$-apt NMR spectrum $\left(100.62 \mathrm{MHz}, \mathrm{C}_{6} \mathrm{D}_{6}, 298 \mathrm{~K}\right)$ of $\mathrm{Rh}\left(\eta^{3}-\mathrm{CH}_{2} \mathrm{CHCHPent}\right)\left\{\kappa^{2}-\mathrm{P}, \mathrm{P}-\left[\operatorname{xant}\left(\mathrm{P}^{\mathrm{i}} \mathrm{Pr}_{2}\right)_{2}\right]\right\}(\mathbf{1 4})$. 

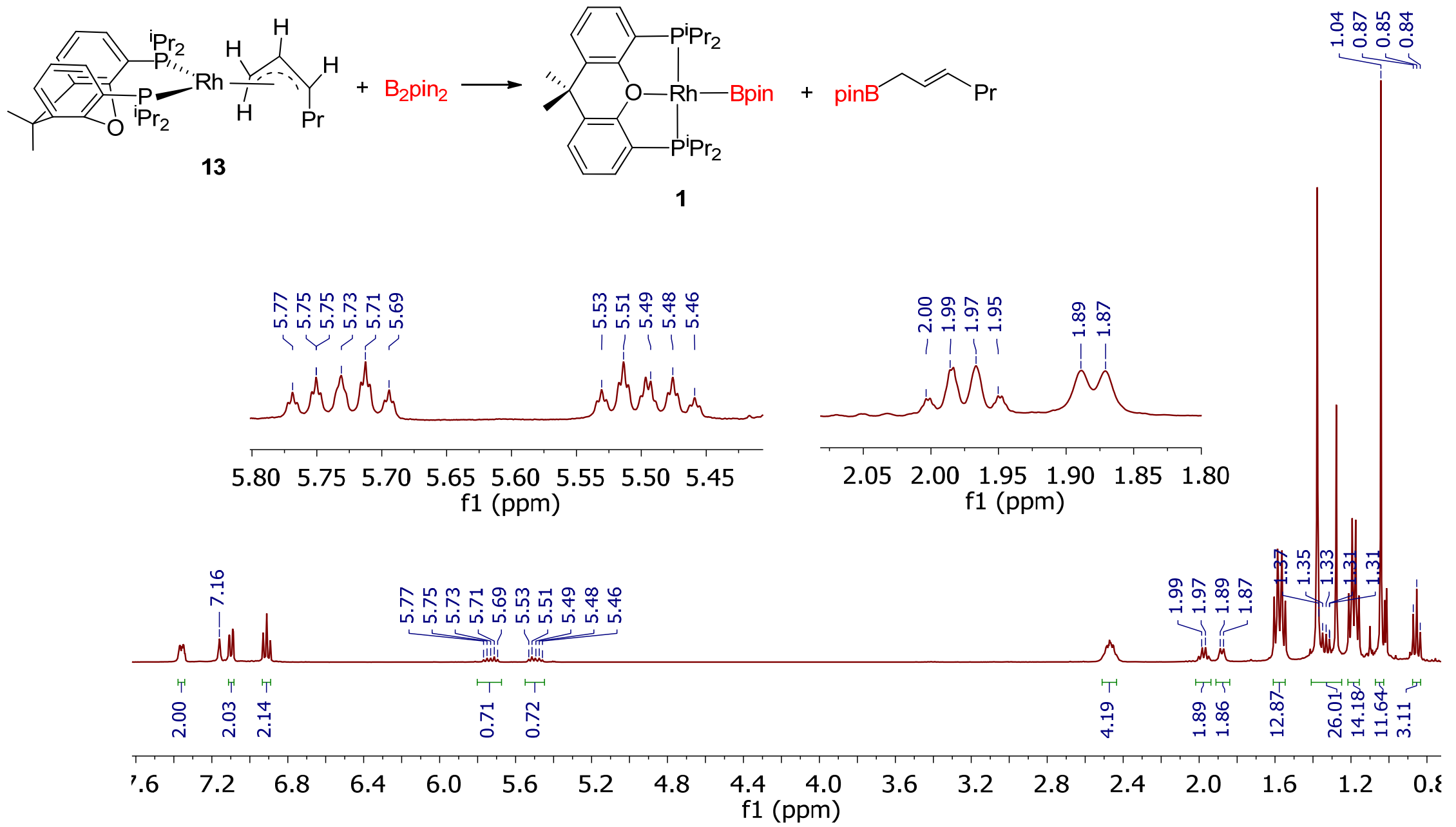

Figure S48. ${ }^{1} \mathrm{H}$ NMR spectrum $\left(400.13 \mathrm{MHz}, \mathrm{C}_{6} \mathrm{D}_{6}, 298 \mathrm{~K}\right)$ of the reaction of $\mathrm{Rh}\left(\eta^{3}-\mathrm{CH}_{2} \mathrm{CHCHCHPr}\right)\left\{\kappa^{2}-\mathrm{P}, \mathrm{P}-\left[\operatorname{xant}\left(\mathrm{P}^{\mathrm{i}} \mathrm{Pr}_{2}\right)_{2}\right]\right\}(\mathbf{1 3})$ with $\mathrm{B}_{2} \mathrm{pin}{ }_{2}$ to give complex 1 and 1-pinacolboryl-2-hexene. 


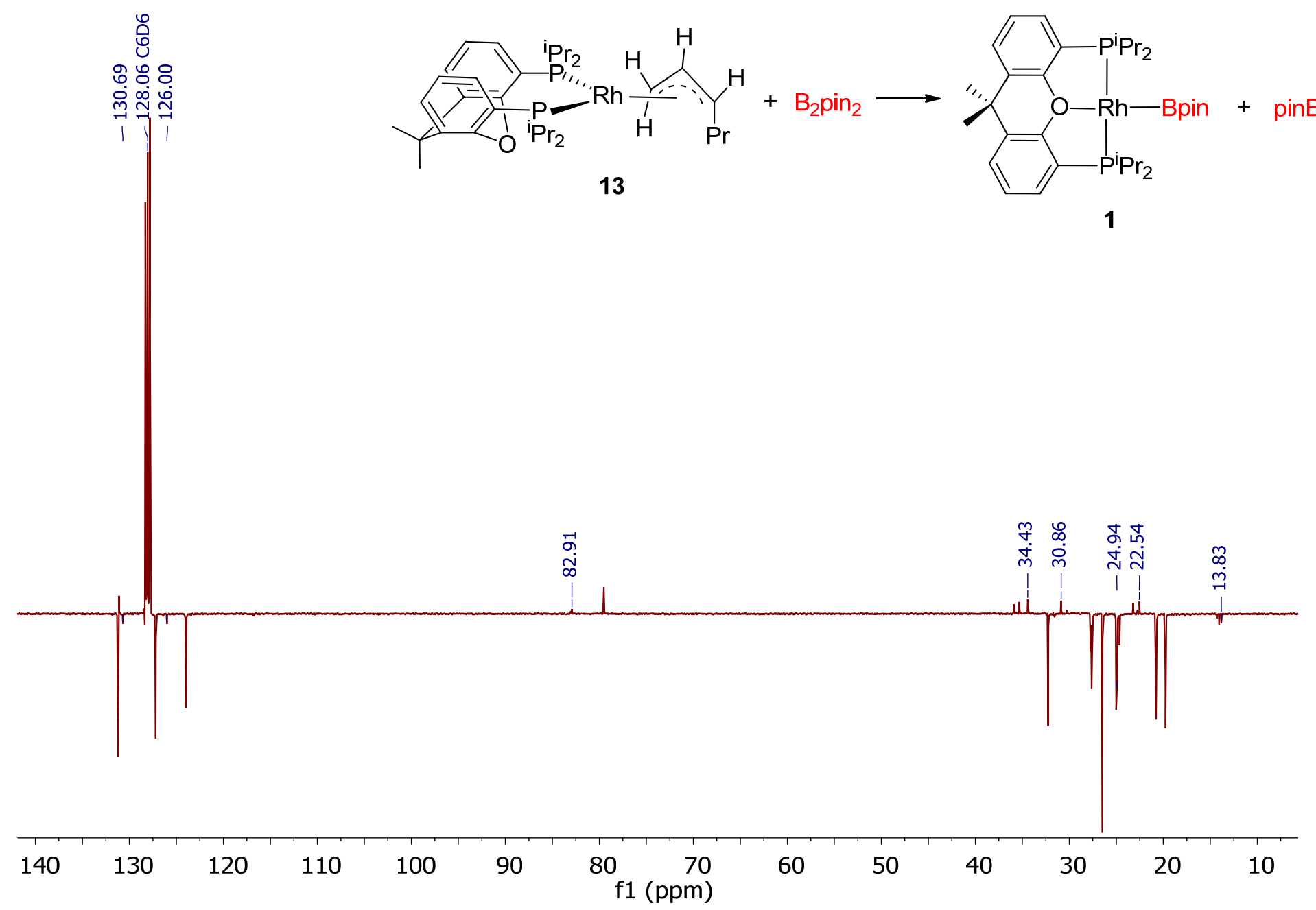

Figure S49. ${ }^{13} \mathrm{C}\left\{{ }^{1} \mathrm{H}\right\}$-apt NMR spectrum $\left(100.62 \mathrm{MHz}, \mathrm{C}_{6} \mathrm{D}_{6}, 298 \mathrm{~K}\right)$ of the reaction of $\left.\mathrm{Rh}\left(\eta^{3}-\mathrm{CH}_{2} \mathrm{CHCHCHPr}\right)\left\{\kappa^{2}-\mathrm{P}, \mathrm{P}-\left[\mathrm{xant}\left(\mathrm{P}^{\mathrm{i}} \mathrm{Pr}\right)_{2}\right)\right]\right\}(13)$ with $\mathrm{B}_{2} \mathrm{pin}_{2}$ to give complex 1 and 1-pinacolboryl-2-hexene. 

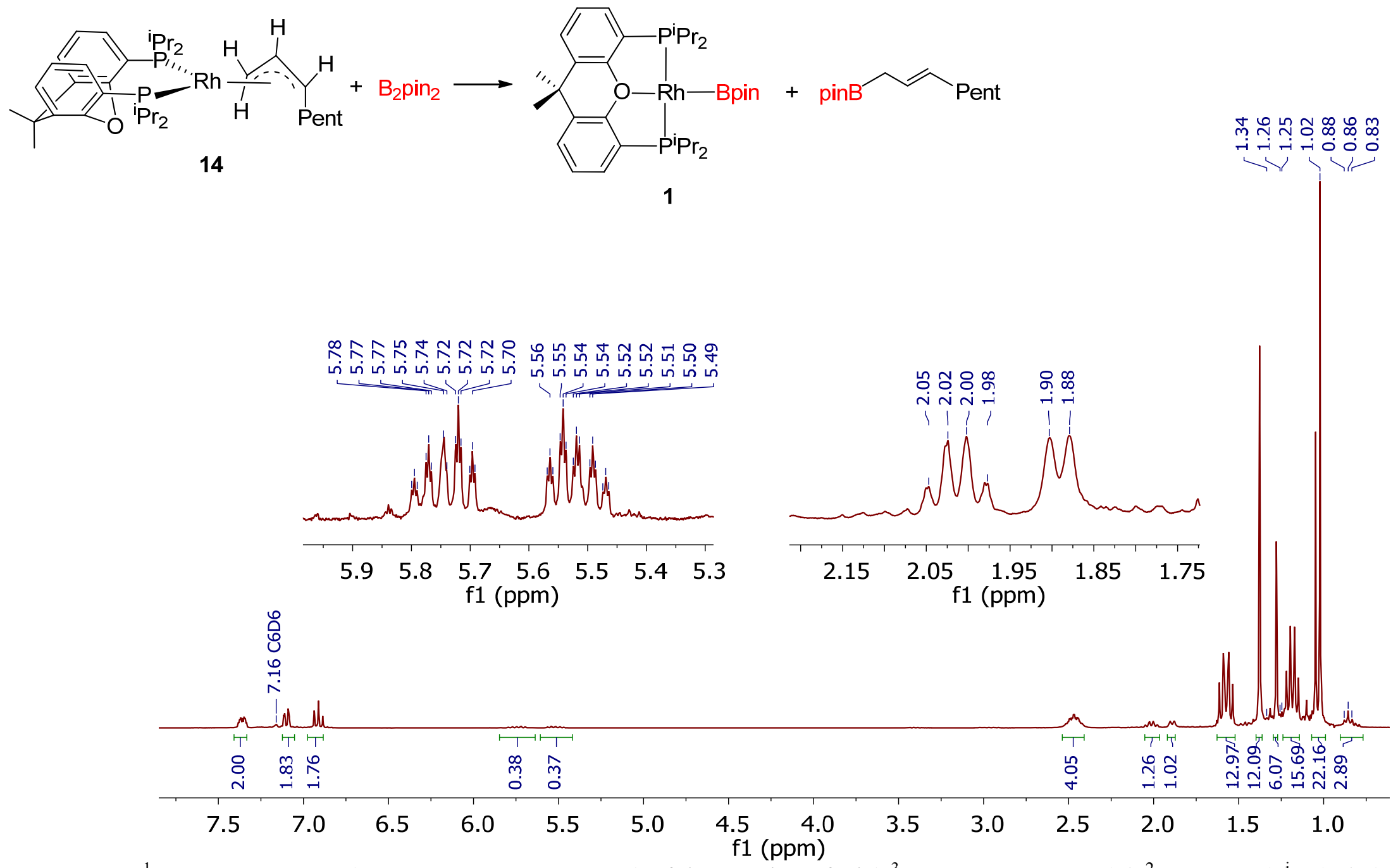

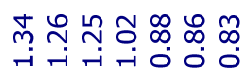

I

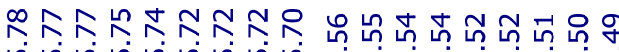

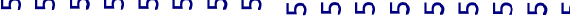

$\begin{array}{lllllll}5.9 & 5.8 & 5.7 & 5.6 & 5.5 & 5.4 & 5.3\end{array}$

f1 (ppm)

Figure S50. ${ }^{1} \mathrm{H}$ NMR spectrum $\left(300.13 \mathrm{MHz}, \mathrm{C}_{6} \mathrm{D}_{6}, 298 \mathrm{~K}\right)$ of the reaction of $\mathrm{Rh}\left(\eta^{3}-\mathrm{CH}_{2} \mathrm{CHCHCHPent}\right)\left\{\kappa^{2}-\mathrm{P}, \mathrm{P}-\left[\mathrm{xant}\left(\mathrm{P}^{\mathrm{i}} \mathrm{Pr}_{2}\right)_{2}\right]\right\}(\mathbf{1 4})$ with $\mathrm{B}_{2} \mathrm{pin}{ }_{2}$ to give complex 1 and 1-pinacolboryl-2-octene. 


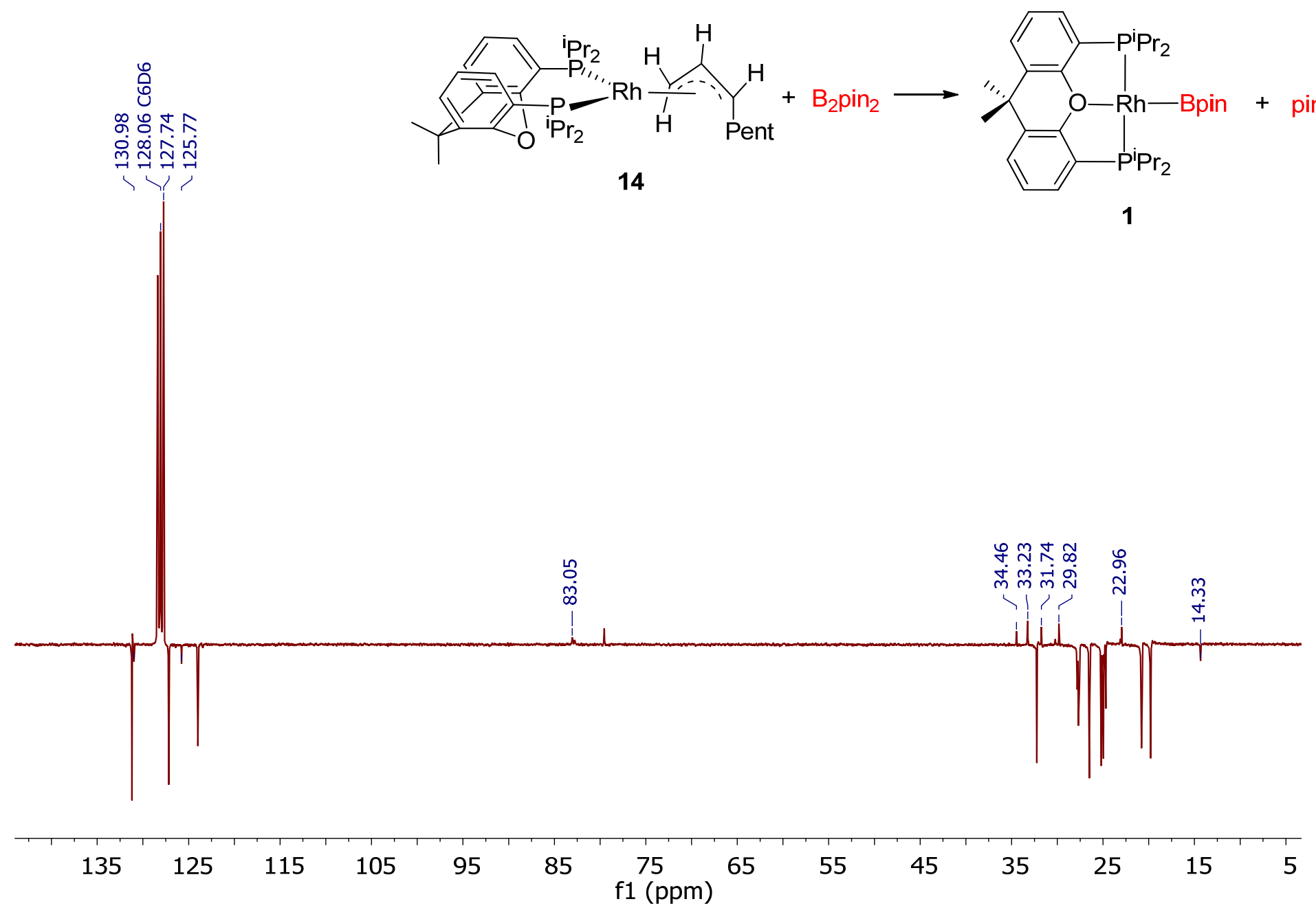

Figure S51. ${ }^{13} \mathrm{C}\left\{{ }^{1} \mathrm{H}\right\}$-apt NMR spectrum $\left(75.48 \mathrm{MHz}, \mathrm{C}_{6} \mathrm{D}_{6}, 298 \mathrm{~K}\right)$ of the reaction of $\mathrm{Rh}\left(\eta^{3}-\mathrm{CH}_{2} \mathrm{CHCHCHPent}\right)\left\{\kappa^{2}-\mathrm{P}, \mathrm{P}-\left[\mathrm{xant}\left(\mathrm{P}^{\mathrm{i}} \mathrm{Pr}_{2}\right)_{2}\right]\right\}(\mathbf{1 4})$ with $\mathrm{B}_{2}$ pin $_{2}$ to give complex 1 and 1-pinacolboryl-2-octene. 
